\title{
Barriers and Facilitators of Seeking Psychological Help for Muslims in the United States
}

\author{
Sarah R. B. Milam
}

West Virginia University, srberkey@mail.wvu.edu

Follow this and additional works at: https://researchrepository.wvu.edu/etd

Part of the Counseling Psychology Commons

\section{Recommended Citation}

Milam, Sarah R. B., "Barriers and Facilitators of Seeking Psychological Help for Muslims in the United States" (2020). Graduate Theses, Dissertations, and Problem Reports. 7783.

https://researchrepository.wvu.edu/etd/7783

This Dissertation is protected by copyright and/or related rights. It has been brought to you by the The Research Repository @ WVU with permission from the rights-holder(s). You are free to use this Dissertation in any way that is permitted by the copyright and related rights legislation that applies to your use. For other uses you must obtain permission from the rights-holder(s) directly, unless additional rights are indicated by a Creative Commons license in the record and/ or on the work itself. This Dissertation has been accepted for inclusion in WVU Graduate Theses, Dissertations, and Problem Reports collection by an authorized administrator of The Research Repository @ WVU.

For more information, please contact researchrepository@mail.wvu.edu. 
Barriers and Facilitators of Seeking Psychological Help for Muslims in the United States

Sarah Milam, M.A.

\author{
Dissertation submitted \\ to the College of Education and Human Services \\ at West Virginia University
}

in partial fulfillment of the requirements for the degree of

Doctor of Philosophy in

Counseling Psychology

\author{
Lisa F. Platt, Ph.D., Chair \\ Monica Leppma, Ph.D., Co-Chair \\ Jeffrey Daniels, Ph.D. \\ Christopher P. Scheitle, Ph.D.
}

Department of Counseling, Rehabilitation Counseling, and Counseling Psychology

\title{
Morgantown, West Virginia
}

2020

Keywords: self-stigma, help seeking, acculturation, Muslim, religious discrimination, ethnic discrimination

Copyright 2020 Sarah R. B. Milam 


\section{ABSTRACT \\ Barriers and Facilitators of Seeking Psychological Help for Muslims in the United States Sarah Milam, M.A.}

Due to increased social, cultural, and political struggles for Muslims in the United States (MUS), affordable, accessible, and culturally appropriate mental health care is important for these individuals (Aloud \& Rathur, 2009). MUS are an underrepresented group that use mental health services at low rates (Ciftci, Jones, \& Corrigan, 2013; Khan, 2006). MUS, especially those with mental health concerns, hold a number of stigmatized identities, often including their race and ethnicity, religion, and mental health status that can result in discrimination (Ciftci et al., 2013). The purpose of this study was to examine factors related to self-stigma for seeking psychological help in MUS. It was hypothesized that Muslim beliefs and practices would increase self-stigma for seeking psychological help and that this relationship would be mediated by acculturation, perceived religious discrimination, and perceived ethnic discrimination. Structural equation modeling was used to test these hypotheses. The direct path between Muslim practices and selfstigma of seeking psychological help was not statistically significant. None of the hypothesized mediated paths were found to be statistically significant. However, results suggested that greater levels of Muslim practices were associated with lower levels of acculturation and that lower levels of acculturation were associated with higher levels of self-stigma for seeking psychological help in a sample of MUS. A number of demographic variables were found to be associated with the main study variables. These findings are discussed along with implications for counseling psychologists and recommendations for future directions in this area of study 
BARRIERS AND FACILITATORS OF SEEKING PSYCHOLOGICAL HELP

\section{DEDICATION}

This dissertation is dedicated to all seekers of God, mental health, and thriving individual and community lives. 


\section{BARRIERS AND FACILITATORS OF SEEKING PSYCHOLOGICAL HELP}

\section{ACKNOWLEDGEMENTS}

I owe thanks to so many who have supported me through graduate school towards a path of personal flourishing and in completion of this dissertation. First and foremost, thank you to Dr. Lisa Platt, my advisor and dissertation chair. Your guidance through this Ph.D., dissertation, and the start of my professional career has been consistent, foundational, strong, and thoughtful. I am so grateful to have had you as my advisor, chair, and mentor. Thank you for your support, mentorship, consistent encouragement, and belief in me.

I am also so grateful to the rest of my committee: Dr. Daniels, you have been in my court offering a strong education, but also guidance, community, and a safe place to land since day one. Dr. Scheitle, I am beyond grateful to you for your assistance with the data analysis of my dissertation. Thank you for taking the time to teach me the process and help me through it. I could not have done it without you. Dr. Leppma, thank you for your comments and feedback which always serve to strengthen my ideas and writing. I have been lucky to have such a wonderful committee through this process.

I am grateful to the contacts I made through the Muslim Mental Health Conference and for the strong support from those in and connected to the Muslim community across the nation. With your support, my eyes, heart, and mind have been opened in new ways. Thank you for extending me your trust and investing in my research. Without the support of this kind, thoughtful, and giving community, my research would not have been possible.

Thank you to my mentors who have guided me through higher education and in pursuit of strength and purpose. Dr. Karen O'Brien, Dr. Christa Schmidt, Dr. Jennifer Taylor, and Dr. Lisa Platt, I have been lucky to learn and grow from such talented women and leaders. Thank you for your investment in me. 


\section{BARRIERS AND FACILITATORS OF SEEKING PSYCHOLOGICAL HELP}

Thank you to my friends and colleagues at Adventure WV and the Carruth Center who gave meaning to my learning throughout graduate school. You made the process fun and purposeful and have allowed my passion to come alive. You have also been my community and made WV feel like home.

I am incredibly grateful to my cohort. I have been blessed to journey through this program with 5 of the most amazing people and future psychologists. A group of incredibly talented, passionate, and unique individuals, we all became stronger through each other. You all let me into your hearts, and have found a lasting place in mine.

I am grateful to my large, loving, and invested family who has always been in my court and encouraged me to pursue my passion with purpose. My family is a treasure and I have been blessed beyond measure through their love and support. Listing each of them and their extensive contributions here would not be possible because they are each so woven into the fabric of who I have become, so I will save special thanks for each of them in person. However, I must acknowledge the support of my incredible husband, partner, friend, and safety net: Josh Milam, words are not enough. I love you.

Finally, I am grateful to God. All of my work springs from a well of His love for all people and I feel privileged to be a part of his story of healing. 


\section{TABLE OF CONTENTS}

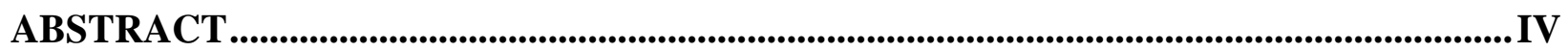

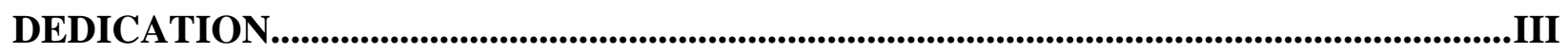

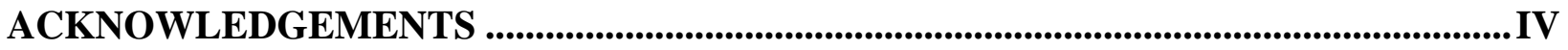

CHAPTER 1: INTRODUCTION AND LITERATURE REVIEW .......................................... 1

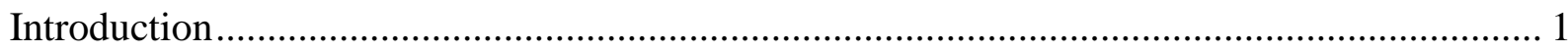

Psychological Help-Seeking Model....................................................................... 1

Muslim Religiosity, Beliefs, and Practices .................................................................. 5

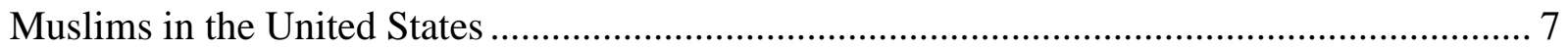

Muslim Mental Health Considerations ........................................................................ 9

Acculturation Considerations for Muslims Living in the U.S. ..................................... 13

Discrimination toward the American Muslim Community ............................................ 16

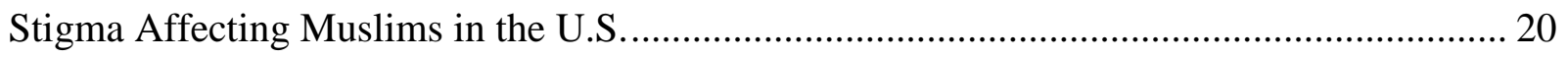

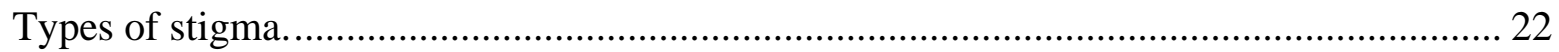

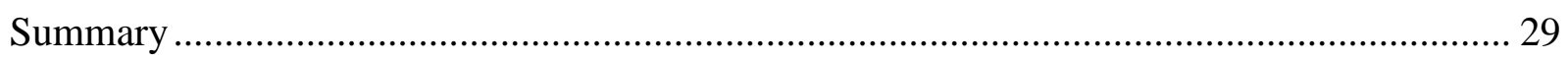

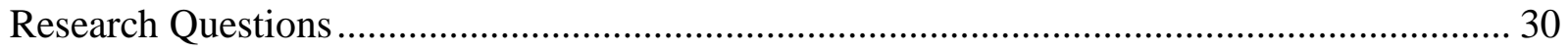

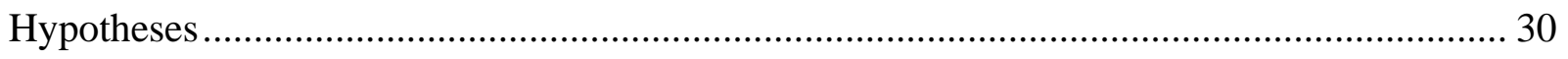

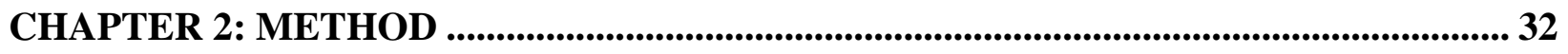

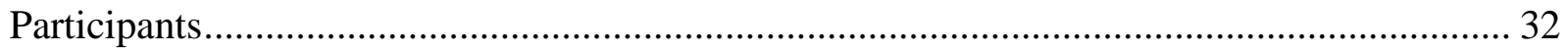

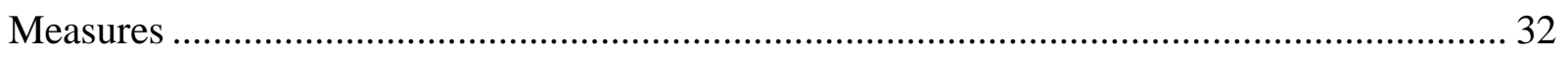

Self-Stigma of Seeking Help Scale (SSOSH; Vogel et al., 2006).................................. 33 
Acculturation Rating Scale for Arabic Americans-II, Attraction to Arabic Culture Subscale

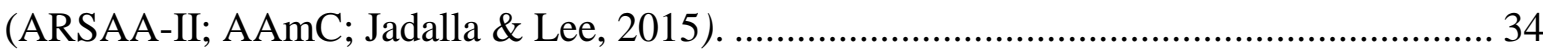

Short Muslim Practice and Belief Scale (S-MPBS; AlMarri et al., 2009). ....................... 37

Brief Perceived Ethnic Discrimination Questionnaire - Community Version Lifetime

Exposure Scale (PEDQ-CV; Brondolo et al., 2005)..................................................... 39

Religious Discrimination Scale (RDS; Kawika Allen et al., 2018)................................ 41

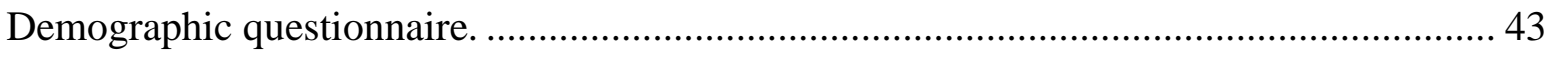

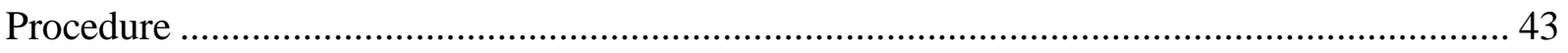

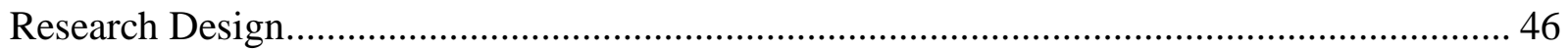

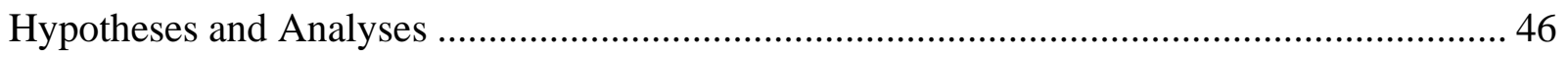

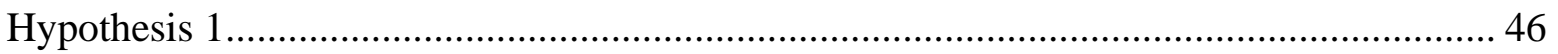

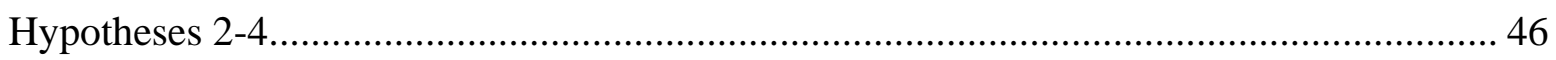

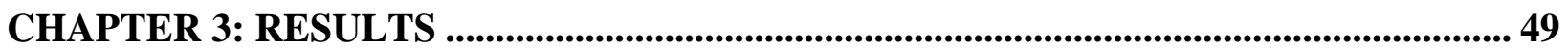

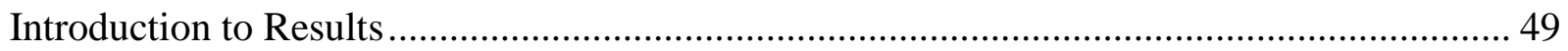

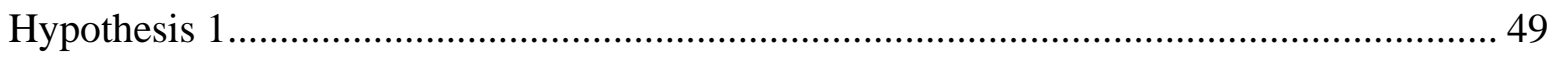

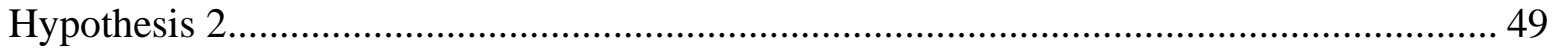

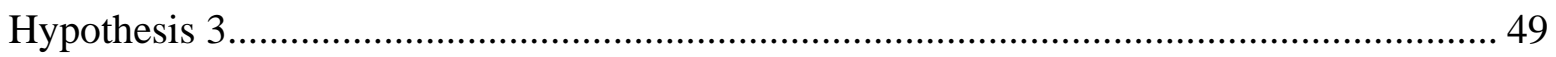

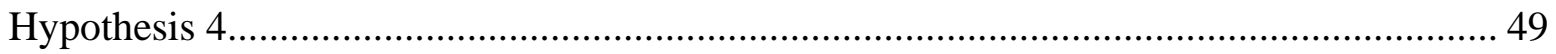

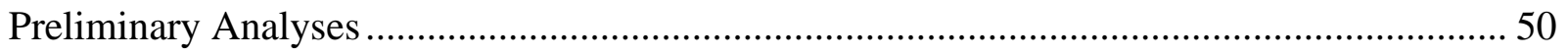

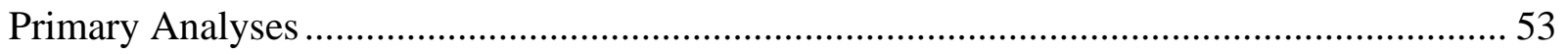

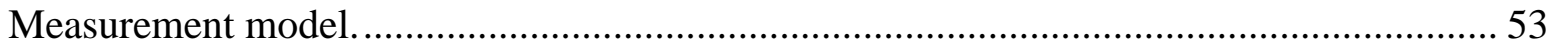

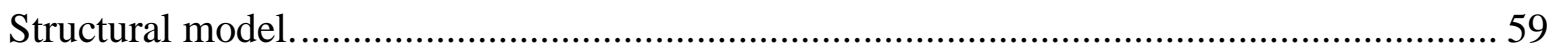

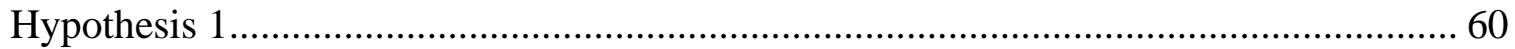




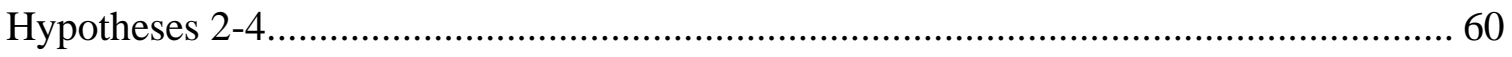

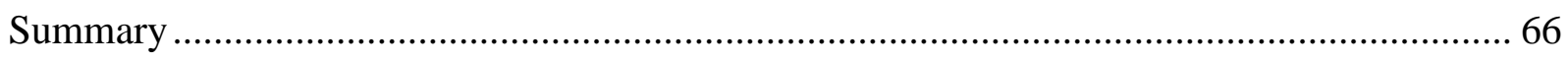

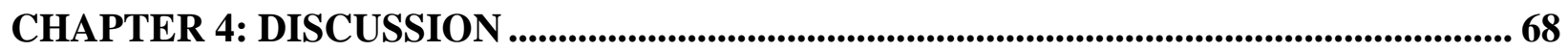

Discussion of the Direct Path between Muslim Practices and Self-Stigma (Hypothesis 1)..... 68

Discussion of the Hypothesized Mediated Paths (Hypotheses 2-4) ..................................... 71

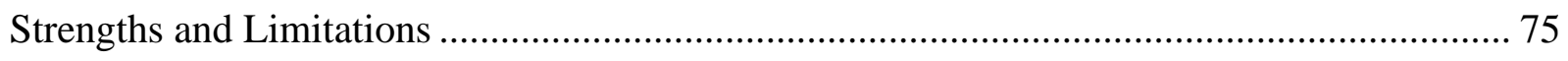

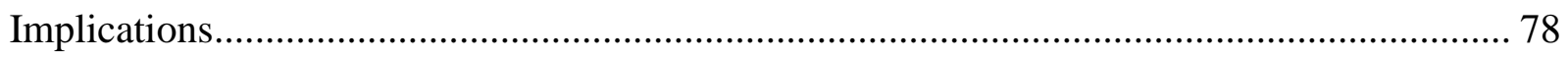

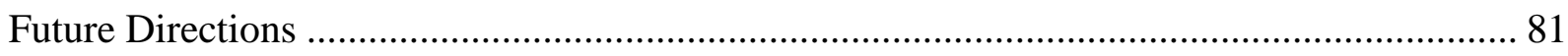

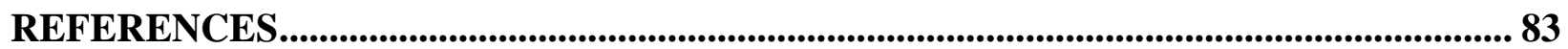

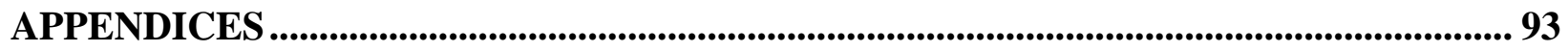

Appendix A: Self-Stigma of Seeking Help Scale .................................................... 94

Appendix B: Acculturation Rating Scale for Arabic Americans-II - Attraction to American

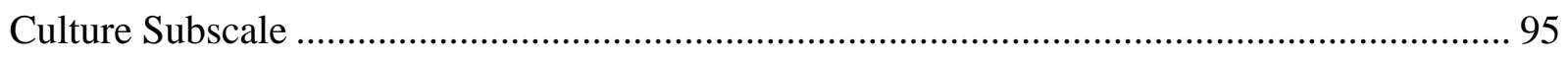

Appendix C: Short Muslim Practice and Belief Scale .................................................... 96

Appendix D: Brief Perceived Ethnic Discrimination Questionnaire - Community Version

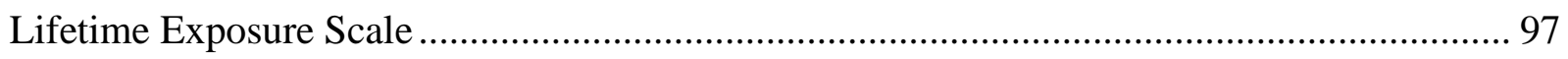

Appendix E: Religious Discrimination Scale ........................................................ 99

Appendix F: Demographic Questionnaire .......................................................... 100

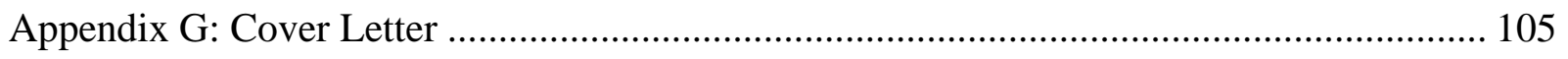

Appendix H: Informed Consent Form ................................................................ 107

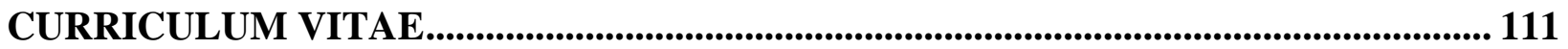




\section{CHAPTER 1: INTRODUCTION AND LITERATURE REVIEW}

\section{Introduction}

Due to increased social, cultural, and political struggles for Muslims in the United States (MUS), affordable, accessible, and culturally appropriate mental health care is important for these individuals (Aloud \& Rathur, 2009). MUS are an underrepresented group that use mental health services at low rates (Ciftci, Jones, \& Corrigan, 2013; Khan, 2006). MUS, especially those with mental health concerns, hold a number of stigmatized identities, often including their race and ethnicity, religion, and mental health status that can result in discrimination (Ciftci et al., 2013). Stigma, the belief of being substandard due to a personal or physical characteristic deemed socially undesirable, is one of the predominant barriers related to seeking mental health treatment (Vogel et al., 2017; Vogel, Wade, \& Haake, 2006). While societal stigma is thought to influence one's decision to seek help, it is likely that self-stigma for seeking psychological help also plays a role. Self-stigma for seeking psychological help occurs as result of internalizing societal stigma and discrimination (Ciftci et al., 2013; Vogel et al., 2017). The purpose of this study is to examine factors related to self-stigma for seeking psychological help in MUS. Specifically, it is hypothesized that perceived religious and ethnic discrimination, acculturation, religiosity, and demographic variables are related to self-stigma for seeking psychological help in a sample of MUS.

\section{Psychological Help-Seeking Model}

Research examining the unique mental health needs of Muslim individuals living in the United States (U.S) has been sparse overall (Abuelezam, El-Sayed, \& Galea, 2017; Abu Raiya, Pargament, Stein, \& Mahoney, 2007); however, due to increased social, cultural, and political struggles for this population, affordable, accessible, and culturally acceptable mental health care 


\section{BARRIERS AND FACILITATORS OF SEEKING PSYCHOLOGICAL HELP}

is important (Aloud \& Rathur, 2009). Yet, mental health services are used at low rates by this group (Aloud \& Rather, 2009; Ciftci, Jones, \& Corrigan, 2013; Khan, 2006; Martin, 2015). It is likely that this is due, in part, to a number of related factors including perceived discrimination, acculturation, religiosity, and self-stigma associated with seeking psychological services. This study examines the nature of the relationship between Islamic beliefs and practices and selfstigma of seeking psychological help, with acculturation and perceived ethnic and religious discrimination serving as potential mediator variables in that relationship. The following literature review presents research on each variable (perceived discrimination, acculturation, religiosity, and self-stigma for seeking psychological help) as it relates to mental health treatment utilization for MUS.

Aloud and Rathur (2009) proposed a model of mental health help-seeking pathways and modifying factors among Arab Muslim populations (Help-Seeking Pathways of Arab Muslims, HSPAM; see figure 1). The HSPAM model was developed by applying literature related to Arab and Muslim cultural values to preexisting help-seeking theories and models (Aloud \& Rather, 2009). The little research conducted using the Arab Muslim population has demonstrated that cultural and religious dimensions play a key role in seeking and accessing formal mental health services (Aloud \& Rather, 2009). They noted that help-seeking behaviors for Arab Muslim individuals can be assessed in three stages: 1) problem awareness and recognition, 2) decision to seek help, and 3) service selection. In the HSPAM model, Arab and Islamic culture and demographic factors (i.e., religion, gender, education, age, and income) operate on each of these stages. In addition, a number of related factors operate on individual stages. Cultural and traditional beliefs and definitions of mental illness, knowledge of mental health problems and treatment, and Arab health styles influence stage one (problem recognition). Perceived societal 


\section{BARRIERS AND FACILITATORS OF SEEKING PSYCHOLOGICAL HELP}

stigma towards mental illness, negative attitudes towards provider influence, and availability of alternative resources such as family and friends influence stage two (decision to seek help).

Stage three (service selection) is influenced by acculturation level, awareness of formal services, use of informal resources, and economic and institutional barriers (Aloud \& Rather, 2009).

Aloud and Rather (2009) investigated dimensions of the HSPAM model using an exploratory, descriptive-associational study to quantitatively assess Arab Muslim help-seeking attitudes. Participants were 285 Arab Muslim community members and college students who were associated with Islamic groups. Informal focus groups with members from the Arab Muslim community, a panel of experts, and pilot testing were used to evaluate the cultural and content validity of the measures used. Respondents reported less favorable attitudes towards using formal mental health services and significant shame associated with seeking mental health help. Participants also reported low levels of knowledge and familiarity regarding mental illness and formal services types or providers (Aloud \& Rather, 2009).

Cultural beliefs about mental health, familiarity with formal mental health services, perceived societal stigma, and help-seeking preferences predicted Arab Muslim attitudes towards formal mental health services (Aloud \& Rather, 2009). Cultural and traditional beliefs regarding mental health were related to increased shame and decreased knowledge regarding seeking formal mental health services. Participants reported that they were more likely to seek mental health help from family doctors, family members, and religious leaders rather than mental health professionals. Help-seeking preference was related to socioeconomic status, nationality, occupation, and type of health insurance but was unrelated to gender, age, and education (Aloud \& Rather, 2009). Those born in the United States were more likely than foreign born Arab Muslims to seek help from formal mental health professionals, family, and friends, suggesting 
that acculturation does play a role in help-seeking behavior. Other than length of time living in the U.S., no other demographic variables were related to help-seeking attitude. These findings provide initial evidence for the HSPAM model, although little additional quantitative research has been conducted to validate the components of this model (Aloud \& Rather, 2009). The present study will test the relationships between demographic factors, perceived ethnic and religious discrimination, acculturation, and Islamic religious beliefs and practices with selfstigma for seeking psychological help in order to quantitatively assess aspects of the HSPAM model for a diverse group of MUS (Aloud \& Rather, 2009).

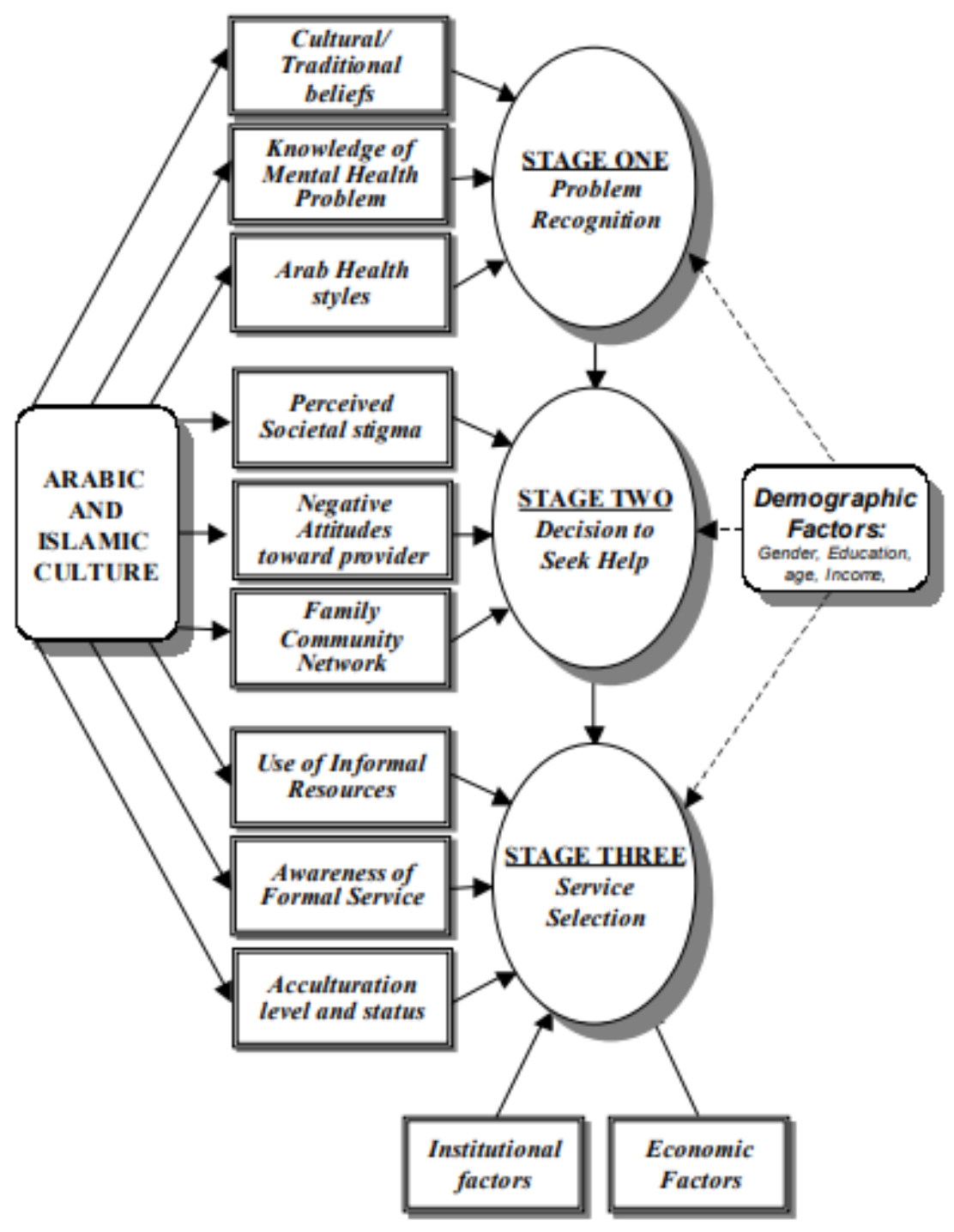




\section{BARRIERS AND FACILITATORS OF SEEKING PSYCHOLOGICAL HELP}

Figure 1. Mental health help-seeking pathways and modifying factors among Arab Muslim populations. Aloud, N., \& Rathur, A. (2009). Factors affecting attitudes towards seeking and using formal mental health and psychological services among Arab Muslim populations.

Journal of Muslim Mental Health, 4, 79-103. http://dx.doi. org/10.1080/15564900802487675

\section{Muslim Religiosity, Beliefs, and Practices}

The word Muslim is used to denote adherents of the religion Islam and translates to "one who submits to the will of Allah" (Ali, Liu, \& Humedian, 2004, p. 636). Allah is the word used by Muslims to describe the God of all humanity (Ali et al., 2004). Muslims believe that Islam began in the $7^{\text {th }}$ century when Muhammed ibn Abdullah, a businessman from Mecca, was meditating and fasting regarding the condition of religion in Arabian society. During this time and subsequently over the next 22 years, the words of the Holy Qur'an were revealed to him from Allah through the angel Gabriel (Ali et al., 2004). Muhammed then preached the message of God to the people of Arabia for the rest of his life and is known as the major prophet of Islam (Ali et al., 2004). The Qur'an is believed by most Muslims to contain universal guidance that extends beyond the $7^{\text {th }}$ century context in which it was given to current and future Muslims. The Qur'an is looked to for direction and is thought by believers to hold answers to questions and crises in life. In addition to the Qur'an, Muslims obtain guidance from the hadith and Sunnah, collections of traditions and sayings from the prophet Muhammed (Ali et al., 2004; Kelly, Aridi, \& Bakhtiar, 1996).

The practice of Islam is quite diverse and varies based on many factors such as culture, religious sect, and nationality (Ali et al., 2004). Even with such variation, most Muslims acknowledge the five pillars of Islam which are specific acts of worship called for in the Qur'an (Kelly et al., 1996). The first pillar of Islam is the belief (iman) that there is only one God, 


\section{BARRIERS AND FACILITATORS OF SEEKING PSYCHOLOGICAL HELP}

Allah, and that Muhammed was his last and final prophet. This constitutes the primary statement of faith which Muslims must recite in order to convert to Islam (Ali et al., 2004). Prayer (salat) is the second pillar of Islam and involves provisions specifying the times and methods Muslims are to follow while praying (Ali et al., 2004). Zakat, the third pillar of Islam, specifies that Muslims give money, or alms, annually as a form of worship and a way to remedy social inequalities (Ali et al., 2004). The fourth pillar of Islam is a prescription for fasting (sawm) during the month of Ramadan (the $9^{\text {th }}$ month of the lunar calendar) in which Muslims abstain from eating, drinking, and sex from sunrise to sunset. During fasting, Muslims continue their daily activities but also participate in self-reflection, spiritual reflection, and contemplation of the condition of the poor and hungry (Ali et al., 2004). The fifth pillar of Islam (Hajj) is a pilgrimage to Mecca which is to occur once during a Muslims lifetime. The pilgrimage is expected only for those Muslims who can afford it and occurs only once per year during the $10^{\text {th }}$ month of the lunar calendar. While on pilgrimage, Muslims perform a series of rituals to commemorate Abrahamic lessons (Ali et al., 2004).

In addition to the five pillars of Islam, there are also a number of other foundational practices that apply to most Muslims, although many MUS hold differing views regarding the method or extent to which Islamic religious observance can or should be accommodated to mainstream American culture (Kelly et al., 1996). For example, dietary restrictions of Islam include restraints against eating pork or consuming alcohol. In addition, most Islamic communities hold customs regarding gender roles, dress, and family values, although these differ per community. For example, Islam prescribes modest dress for both men and women. This prescription is often most obvious for Muslim women (Ryan, 2011). Interpretations of modesty for Muslim women often depend on cultural or family traditions and range from simple modest 


\section{BARRIERS AND FACILITATORS OF SEEKING PSYCHOLOGICAL HELP}

clothing, to wearing the hijab (head covering), to full covering (burqa) (Ali et al., 2004). Many Muslim communities also have expectations for gender relations, valuing heterosexual marriage and discouraging close ties between men and women outside of marriage (Ali et al., 2004). Islamic religiosity and culture are frequently thought to play an important role in the mental health concerns, behaviors, and service selections of Muslims (Bhui, King, Dein, \& O’Connor, 2008; Ciftci et al, 2013).

\section{Muslims in the United States}

The modern cultural identity of MUS is multidimensional and includes an interplay of religion, culture, ethnicity, and race that varies in significance for each individual (Jasperse, Ward, \& Jose, 2012). A heterogeneous group, Muslims in America demonstrate considerable diversity on a number of dimensions such as Islamic tradition (e.g., Sunni, Shi'ite, Sufi), country of origin (e.g., native born or immigrant), pathway to Islam (e.g., convert to Islam or born into the faith), race (e.g., African American, Arab, South Asian, White, etc.), ethnicity (representing over 80 countries) socioeconomic status, and language (Amer \& Bagasra, 2013; Read, 2008; United States Department of State, 2009). There is no official governmental count of the U.S. Muslim population because the U.S. Census Bureau does not include survey questions regarding religion; however, the Pew Research Center estimates that in 2017 there were approximately 3.45 million MUS (about 1.1\% of the total U.S. population) (Mohamed, 2018). Some scholars estimate slightly higher population numbers of six to seven million Muslims living in the U.S. (Amri \& Bemak, 2013; Nadal et al., 2012). The MUS population is expected to grow by approximately 100,000 people per year since 2011 , largely due to migration and higher fertility rates among Muslims (Mohamed, 2018). 


\section{BARRIERS AND FACILITATORS OF SEEKING PSYCHOLOGICAL HELP}

According to the Pew Research Center on Religion and Public Life (2018), 58\% of U.S. Muslim adults are foreign-born with many having immigrated following the 1965 Immigration and Nationality Act which made immigration more accessible to individuals from Asia, Africa, and other non-European regions. Over half of U.S. born American Muslims are the children of Muslim immigrants (Amri \& Bemak, 2013). MUS, originating from over 80 countries, are the most ethnically diverse population in the world (Read, 2008). The majority of foreign-born MUS are from South Asia (20\%) including Afghanistan, Pakistan, India, Bangladesh, and Iran. MUS also originate from the Middle East and North Africa (14\%), and the Asian Pacific (13\%), with smaller populations from Sub-Saharan Africa (5\%), Europe (2\%), and the Americas (excluding the U.S., 2\%) (Pew Research Center on Religion and Public Life, 2018). Due to the diverse regional backgrounds of foreign-born MUS, the languages spoken by this heterogeneous group are varied and can include English, Arabic, Farsi, Turkish, or Indonesian or African languages (Martin, 2015). In addition, most Non-Arab Muslims know a minimum amount of Formal Arabic, as prayer includes recitation of the Qur'an in Arabic, the original revelation language of Islam (Martin, 2015). The largest percentage of the Muslim immigrant population in the U.S. identifies as Sunni Muslim (approximately half) while 22\% do not identify as a particular sect and 16\% identifies as Shi'a Muslim (Amri \& Bemak, 2013). According to the U.S. Department of State (2009), the highest populations of American Muslims are located in California, New York, Illinois, New Jersey, Indiana, Michigan, Virginia, Texas, Ohio, and Maryland.

There are many similarities between foreign-born and U.S. born MUS including similar levels of religious practice such as masjid attendance, commitment to eating halal food, or fasting during Ramadan (Pew Research Center on Religion and Public Life, 2018). Additionally, 


\section{BARRIERS AND FACILITATORS OF SEEKING PSYCHOLOGICAL HELP}

both native and immigrant Muslim women reported they were likely to wear head coverings in public at similar rates. However, a number of differences, including racial and ethnic identities and socioeconomic status, arise between the groups. Foreign-born Muslims primarily identify as White (45\%), Asian (41\%), and Black (11\%). In contrast, U.S. born Muslims primarily identify as White (35\%), Black (32\%), Hispanic (17\%), and Asian (10\%). In each group, there are a large number of Muslims who identify as White, a category that includes people who are Arab, Middle Eastern, or Persian (Pew Research Center on Religion and Public Life, 2018). In terms of socioeconomic status, Muslim immigrants often fare better than U.S-born Muslims, a finding that may be compounded by preexisting racial wealth disparities in the U.S. For example, Muslim immigrants, who are more likely to identify as White or Asian, are also almost twice as likely to own a home or have a college degree or have a higher household income than their U.S.-born counterparts, who primarily identify as racial minorities (Pew Research Center on Religion and Public Life, 2018).

\section{Muslim Mental Health Considerations}

Muslims in the U.S. face a number of threats to mental health due to both individual, contextual, and societal factors which include acculturation difficulties, racism, Islamophobia, and discrimination (Amri \& Bemak, 2013). MUS may also experience difficulties related to trauma due to a number of factors including interpersonal violence, community violence, political unrest, or discrimination (Amri \& Bemak, 2013). For example, many Muslim immigrants to the U.S. have experienced war or sociopolitical strife resulting in clinical problems such as posttraumatic stress, anxiety, and depression (Ali et al., 2004). MUS regularly report feelings of anxiety regarding their status in the country due to anti-Muslim sentiment and fear of discrimination or hate crimes directed at them (Ali et al., 2004). Aside from culture 


\section{BARRIERS AND FACILITATORS OF SEEKING PSYCHOLOGICAL HELP}

specific concerns, MUS also face generally common mental health concerns including substance abuse, domestic violence, anxiety, and depression (Amri \& Bemak, 2013). Mental health difficulties such as these can have a negative impact on one's ability to function in multiple domains including employment, family relations, and societal functioning (Amri \& Bemak, 2013).

Given these concerns, culturally competent mental health services could benefit MUS, and yet these services are used at low rates by this population (Ali \& Milstein, 2012; Aloud \& Rather, 2009; Ciftci, Jones, \& Corrigan, 2013; Khan, 2006). Some propose that this is related to cultural definitions of mental health and appropriate treatment. In Arab Muslim culture, for example, mental illness is frequently attributed to possession by supernatural forces such as demons (jinn), the evil eye (Nathla), or magic (Seher) (Aloud \& Rather, 2009). Less common is the belief that mental illness is attributable to genetic factors (Youssef \& Deane, 2006). Arab Muslims are more likely to somaticize mental health concerns rather than distinguish mental or emotional problems from physical problems (Aloud \& Rather, 2009). Likewise, Arab Muslims often prefer informal, traditional, and religious healing methods over biomedical services or counseling which they are often less familiar with (Aloud \& Rather, 2009). Often, Arab Muslims, unfamiliar with the mental health field, express uncertainty regarding types of mental health professionals and types of services offered. Arab Muslims tend to tolerate mental health problems longer rather than seek timely treatment for a number of reasons including that it is viewed as negative to complain about pain (Aloud \& Rather, 2009).

Although Islamic religious beliefs and practices regarding health and illness have a number of contextual differences within and between Muslim groups, there are also important commonalities that should be considered (Ciftci et al., 2013). A basic and core belief of Islam is 


\section{BARRIERS AND FACILITATORS OF SEEKING PSYCHOLOGICAL HELP}

the existence of one God, referred to as Allah in Arabic. Allah is believed to cause everything, including prosperity or illness. Depending on the context, illness can be seen as a method to connect with Allah, or be an opportunity to cleanse, purify, and balance one physically, emotionally, or spiritually (Ciftci et al., 2013). Additionally, mental illness can be seen as a test or punishment from Allah (Aloud \& Rather, 2009).

In one study using participatory action research, 13 focus groups were conducted at area mosques in southeast Michigan to understand American Muslim views on healing (Padela, Killawi, Forman, DeMonner, \& Heisler, 2012). Participants maintained a God-centric view of healing in which healing was accessed directly through prayer or reading the Qur'an or indirectly through people such as imams, doctors, family, and other community members. Religious and spiritual coping can be a powerful way to cope with and give life meaning and constitutes an important cultural practice for many (Bhui et al., 2008). Prayer, seeking guidance, and receiving comfort from God and one's spiritual communities have been reported by Muslims (and individuals from other faith traditions) to be common, helpful means to cope with and make sense of difficult circumstances (Bhui et al., 2008). In Muslim American communities, family and friends are often relied upon for emotional, physical, and spiritual support (Ali et al., 2004). Participants noted that the imam is frequently used to fulfill psychological counseling roles within the community in place of trained mental health providers even though it was noted that many imams lack formal education or training in counseling or therapy (Padela et al., 2012). Likewise, mental health providers often lack appropriate cultural understanding and skills regarding the important role religion and spirituality can play for Muslims in coping and healing from mental and physical health concerns (Bhui et al., 2008). It is recommended that collaborations between mental health providers and imams could help foster greater cultural 


\section{BARRIERS AND FACILITATORS OF SEEKING PSYCHOLOGICAL HELP}

understanding for mental health care providers and instill increased counseling and referral skills to imams (Bhui et al., 2008).

Although collaboration is encouraged, barriers exist that influence help-seeking for this population. In a qualitative study investigating health care values of 25 Muslims in Israel and the United States, many participants noted suspicion and doubt regarding Western psychology, often viewing it as secular and antireligious (Abu Raiya et al., 2007). In addition, participants expressed negative reactions to the term mental health in the interviews, frequently noting fear of being stigmatized or being thought of as crazy (Abu Raiya et al., 2007). Islam is seen as the source of healing and strength in many Muslim communities, and often Muslims struggling with mental health concerns are stigmatized as having lost their faith in God or lost their way by others in their religious community (Amri \& Bemak, 2013). When formal mental health care is delayed or avoided, especially for more serious presenting concerns such as psychosis, bipolar disorder, major depressive disorder, and anxiety disorders, it can result in worse outcomes (Clement et al., 2015). Stigma is involved in reducing help-seeking for mental health services (Clement et al., 2015). These findings suggest the need for greater understanding of barriers and facilitators for mental health treatment seeking for MUS to improve access to and utilization of culturally informed care.

Mental health beliefs as well as therapy seeking behaviors vary for Muslims in the U.S. based on many factors including religiosity, acculturation, ethnicity, culture, and stigma (Ali et al., 2004; Ali \& Milstein, 2012; Ciftci et al., 2013). Cultural beliefs function both to define societal beliefs regarding what constitutes an illness and how to respond and treat the illness. Cultures define mental health differently such that in one society a concern may be viewed as mental illness whereas in another society it could be viewed as no more than a common hassle 


\section{BARRIERS AND FACILITATORS OF SEEKING PSYCHOLOGICAL HELP}

(Aloud \& Rather, 2009). It is likely that Muslim religiosity, acculturation, and discrimination play a role in engendering self-stigma of seeking psychological help for MUS and these relationships need to be further understood.

\section{Acculturation Considerations for Muslims Living in the U.S.}

Over half of MUS are immigrants (Pew Research Center on Religion and Public Life, 2018) and approximately half of U.S. born Muslims are children of immigrants (Amri \& Bemak, 2013). Acculturation, the process of adoption and immersion of the adoptive society or retention of the ethnic society of origin, has been a well-documented influence of attitude towards seeking mental health services in a number of immigrant populations in the U.S. (Aloud \& Rathur, 2009). Acculturation outcomes can be impacted by factors such as country of origin, sociopolitical forces, amount of time in the U.S., reason for immigration, education level, socioeconomic status, English language proficiency, and quality of social support (Amri \& Bemak, 2013). Socioeconomic status, including access to health insurance, is highly related to access and utilization of formal mental health treatment (Aloud \& Rather, 2009). Likewise, acculturation processes may be affected by factors related to mental health including trauma or discrimination faced either prior to or following immigration (Amri \& Bemak, 2013).

Acculturation to a new country, especially one with a history of oppression towards Muslims and racial minorities, poses a potential stressor for MUS (Abuelezam et al., 2017).

An interplay between adoption of the dominant culture and retention of the ethnic society of origin, acculturation, whether due to one's direct immigration or that of a close family member, often affects the entire family system (Amri \& Bemak, 2013). For example, children may be parentified as they often learn English more quickly than their parents and thus must speak for their parents or make important family decisions in the place of their parents (Amri \& 


\section{BARRIERS AND FACILITATORS OF SEEKING PSYCHOLOGICAL HELP}

Bemak, 2013). Often education and job skills from one's native country do not transfer to the U.S. job market, creating difficulty earning a living and creating a new life. As a result, women frequently assume the role of the secondary or primary wage earner, a role they may not have previously occupied. This could upset the balance of power in a patriarchal family system where the male is expected to be the financial provider (Amri \& Bemak, 2013). In addition to changing cultural customs and norms, immigrants often struggle to navigate the complicated social services, educational, and legal systems in the U.S. (Amri \& Bemak, 2013). Each of these experiences represents significant stressors that could result in feelings of marginalization, isolation, and stigma, along with mental health concerns.

Muslim families who are less acculturated to U.S. norms are often more collectivistic with a focus on respect of parents and elders and a value of interdependence. Frequently, Muslims are expected to keep personal or family difficulties within the family unit and are discouraged from sharing personal struggles outside the home (Ali et al., 2004). Decisions are expected to be made in consideration of the benefits and detriments to the family and broader Muslim community and not with sole regard to the individual (Ali et al., 2004). These cultural norms are often linked to generational status and acculturation. For example, recent Muslim immigrants to the U.S. are often more religious than their U.S. born children and this can raise conflict over the practice of the faith or mental health norms between parents and children (Ali et al., 2004).

Often in samples less acculturated to Western culture, Muslim individuals attribute mental illness to religious or spiritual forces rather than biomedical or intrapsychic sources. For example, Arab Muslims receiving mental health services in Jordan were more likely to attribute

their problems to the evil eye, magic, envy, or a punishment or test caused by Allah (Al-Adawi et 


\section{BARRIERS AND FACILITATORS OF SEEKING PSYCHOLOGICAL HELP}

al., 2002; Aloud \& Rathur, 2009). Likewise, Arab Muslim individuals often seek out informal or traditional help, such as family, religious leaders, friends, or physicians for mental health problems rather than formal psychological services (Aloud \& Rather, 2009). Scholars have proposed that the hesitancy to seek out formal mental health services by Muslim individuals may be due largely to stigma of mental health services and seekers as well as cultural mistrust of mental health counselors and the mental health system (Ali \& Milstein, 2012; Amri \& Bemak, 2013; Kelly et al., 1996; Ciftci et al., 2013).

In a study of 121 MUS, most respondents indicated that they would prefer a Muslim counselor (52.9\%) to a non-Muslim counselor and $75 \%$ of participants stated that it was very to somewhat important that non-Muslim counselors have an understanding of Islamic values (Kelly et al., 1996). Muslims in the study, especially highly religious Muslims, also tended to hold a more conservative, conventional, and traditional approach to life as compared to the values held by professional counselors (Kelly et al., 1996).

Cultural mistrust is a term used to describe mistrust held by minority groups in the U.S, toward White-dominated institutions including legal and educational systems, job settings, and the government based on a history of racism, discrimination, and oppression (Amri \& Bemak, 2013). Cultural mistrust could explain in-part the hesitancy on behalf of MUS to seek professional mental health care in the U.S. Although not tested empirically, Amri and Bemak (2013) theorized that cultural mistrust of mental health providers on behalf of Muslims living in the U.S. could be a particularly salient barrier in seeking mental health care for those who also experience significant discrimination based on their race or religion. They note in particular a fear held by many Muslims that mental health counselors may both fail to understand their 


\section{BARRIERS AND FACILITATORS OF SEEKING PSYCHOLOGICAL HELP}

unique cultural and religious contexts and also influence the Muslim client in ways that conflict with their religious beliefs and values (Amri \& Bemak, 2013).

In Australia, a sample of Arabic-speaking individuals noted strong concerns regarding confidentiality and lack of trust in service providers (Youssef \& Deane, 2006). Scholars note that stigma attached to mental illness in the Muslim community often results in Muslims preferring to seek support from those in their family or community rather than professional mental health providers (Amri \& Bemak, 2013). It is possible that in addition to social stigma, MUS may experience internalized self-stigma for seeking psychological help and that this process may be exacerbated by acculturation, religiosity, and perceived discrimination. Taken together, it is possible that higher levels of Muslim religiosity could be related to greater experienced religious and ethnic discrimination and lower acculturation, and thereby increase self-stigma for seeking psychological help as proposed in the present study.

\section{Discrimination toward the American Muslim Community}

MUS, members of a religious, and often racial or ethnic minority group, are frequently faced with difficulties including discrimination, prejudice, and stigma that negatively impact their ability and desire to seek psychological assistance when it could be beneficial (Ciftci et al., 2013). Category-based responses, which encompass stereotypes, prejudice, and discrimination, occur when one reacts to another as an interchangeable member of a particular social group (Fiske, 2014). For example, when someone categorizes a Muslim as a "terrorist" they are engaging in a discriminatory category-based response using a false association with extremists due to a nominally shared religion, racial, or national background (Ciftci et al, 2013). This is an incorrect and harmful association. 


\section{BARRIERS AND FACILITATORS OF SEEKING PSYCHOLOGICAL HELP}

Stereotypes, prejudice, and discrimination, while related, each denote a slightly different process. Stereotypes are beliefs about the attributes of group members and assumptions about why those characteristics are grouped together (Fiske, 2014). Although often harmful in practice, stereotypes are used to make connections in the world more manageable and occur through applying one's cognitive expectancies and beliefs to a group, frequently reflecting a social consensus or collective belief system (Fiske, 2014). Stereotypes occur when applying generalizations to particular cases and are often inaccurate, misleading, and harmful (Ciftci et al., 2013). Prejudice occurs when one evaluates another, usually an outgroup member, and reacts to him or her emotionally on the basis of their feeling about that individual's group as a whole (Fiske, 2014). Prejudice entails negative affect and is based on implicit or explicit agreement with derogatory or pejorative stereotypes (Ciftci et al., 2013). Discrimination is the behavioral component of stigma and follows when one acts on stereotypical or prejudicial beliefs in a manner that denies fair treatment to others (Ciftci et al, 2013; Fiske, 2014). Discrimination can take the form of verbal rejection, avoidance, segregation, physical attack, or even killing (Fiske, 2014). These constructs interact. For example, an individual who believes that Muslim men are dangerous or untrustworthy (stereotype) and thus holds negative attitudes towards Muslims (prejudice) may avoid neighborhoods where Muslims live or report them to airport police (discrimination) (Ciftci et al., 2013).

MUS can face discrimination based on racial bias and Islamophobia (Rippy \& Newman, 2006). Following the terrorist attacks of September 11, 2001, MUS became the second highest group, following Jews, to report religiously-based hate crimes which included mosque burnings, bomb threats, physical and verbal attacks, and employment discrimination (Amri \& Bemak, 2013; Rippy \& Newman, 2006). Since the 9/11 terrorist attacks, MUS are also at an increased 


\section{BARRIERS AND FACILITATORS OF SEEKING PSYCHOLOGICAL HELP}

risk for being violently attacked due to their religion (Ghaffari \& Citfci, 2010). In response, many MUS report increased likeliness of suspicion, vigilance, and mistrust of others (Abu Raiya et al., 2007; Rippy \& Newman, 2006). Arab American college students, often assumed to be Muslim, reported increased levels of psychological distress and poorer physical health in response to perceived discrimination based on race and faith (Padela \& Heisler, 2010). Likewise, Arab American mothers in California had increased adverse birth outcomes, associated with discrimination and stress, following the 9/11 terrorist attacks (Lauderdale, 2006).

In addition to overt forms of discrimination, MUS also face subtle forms of discrimination perpetrated by individuals, the media, and institutions (Amri \& Bemak; Nadal et al., 2012). MUS report negative effects due to their religion, ethnicity, or nationality that are sanctioned by the U.S. government including legislation such as the Patriot Act (Amri \& Bemak, 2013) or Executive Order 13769 which banned travel to the U.S. from several predominantly Muslim countries (Liptak \& Shear, 2018). Legislation such as this has resulted in home raids, arrests, detentions, and interrogations of Muslim individuals in the U.S. (Amri \& Bemak, 2013). In a qualitative study examining microaggressions faced by Muslim Americans, participants reported facing religious stereotypes casting Muslims as terrorists, pathologizing Islam, the assumption of religious homogeneity, exoticization of the religion and culture, use of Islamophobic or mocking language, and the implication of being an alien in the U.S. (Nadal et al., 2012). Findings also revealed that Muslim Americans are often cast as violent, evil, or untrustworthy in mainstream U.S. cultural narratives. Narratives like these can increase negative speech directed towards American Muslims such as the use of derogatory comments like "Say hi to Osama” (Nadal et al., 2012, p. 23), as well as negative behaviors including a greater likelihood of being searched at airports. Participants reported instances of people mocking or 


\section{BARRIERS AND FACILITATORS OF SEEKING PSYCHOLOGICAL HELP}

demeaning Islam and its adherents. Muslim American participants also reported often encountering the stereotype that every Muslim is the same with the assumption being that all Muslims are Arabs and practice the religion in the same way, which invalidates and ignores the vast diversity among those who practice Islam (Nadal et al., 2012). Many Muslim Americans face microaggressions based on intersecting marginalized identities including religion, race, ethnicity, and gender, among others. Findings from this study suggest that Islamophobic, racial, and ethnic microaggressions continue to have a discriminatory and harmful effect on MUS (Nadal et al., 2012).

Outward identifiers, such as attire or skin color, have been found to play a role in discrimination. In one study, Muslim women reported greater collective discrimination than their male counterparts due to the way their dress often identifies their religion outwardly (Ryan, 2011). Discourses in politics and the media are often heavily gendered and focus on outward signs of Islam, such as the hijab worn only by women. Often the hijab is prejudicially pointed to as an example that Muslims are a "problematic minority" that refuse to integrate with society (Ryan, 2011). Muslim women are more frequently put in the position of navigating situations in which their religion is automatically disclosed through powerful signifiers of difference such as clothing and physical presentation. This can result in increased prejudice and discrimination directed at Muslim women compared to their male counterparts (Ryan, 2011).

Experiencing stereotypes, prejudice, and discrimination may impact MUS engagement in mental health treatment (Martin, 2015). Often providers are unaware and ill-equipped to provide culturally competent care to this group which may further oppress MUS experiencing discrimination and stigma when attempting to seek psychological help (Martin, 2015). The impact of discrimination on the mental health of MUS is complex, but likely includes self- 


\section{BARRIERS AND FACILITATORS OF SEEKING PSYCHOLOGICAL HELP}

stigma. Ghaffari and Citfci (2010) conducted a study examining the relationship between religiosity and self-esteem in Muslims immigrating to the United States. They noted that although positive in-group membership is often related to higher self-esteem and reduced negative outcomes in the face of discrimination, this is not always the case. They found that perceived discrimination moderates the relationship between religiosity and self-esteem. These results suggest that greater levels of perceived discrimination, regardless of high religious identity and in-group membership, are related to decreases in self-esteem. Thus, perceived discrimination affects the direction and strength of the relationship between religious attitudes and behaviors with self-esteem (Ghaffari \& Citfci, 2010). When faced with perceived discrimination, Muslim immigrants were more likely to turn to and identify more strongly with their religion, although additional coping strategies may be required in order to fully address the negative effects experienced due to prejudice and discrimination as self-esteem was still lower for this group (Ghaffari \& Citfci, 2010). It is possible that stigma plays a role in this effect and requires further investigation.

Muslims in America experience numerous negative effects due to discrimination. One harmful result of stereotyping, prejudice, and discrimination is stigma. Due to the intersection of marginalized identities (race, religion, nationality, mental illness) that MUS with mental health concerns hold, stigma is a particularly relevant topic for this group. In fact, stigma is thought to be a primary barrier preventing MUS from seeking mental health care, and numerous calls exist to further investigate this phenomenon (Ciftci et al., 2013; Vogel et al; 2013; Vogel et al., 2017).

\section{Stigma Affecting Muslims in the U.S.}

Stigma is a societal reaction to perceived negative deviance and is often related to social norm enforcement (Bos, Pryor, Reeder, \& Stutterheim, 2013). It occurs when an attribute or 


\section{BARRIERS AND FACILITATORS OF SEEKING PSYCHOLOGICAL HELP}

characteristic, such as race, mental health status, religion, or gender, is used to denote widespread social disapproval or devaluation, invalidating one's identity (Bos et al., 2013; Vogel et al., 2017). Stigma involves labeling, separation, stereotype awareness, stereotype endorsement, prejudice, and discrimination (Clement et al., 2015). It often exists in systems in which social, economic, and political power are applied to the disadvantage of members of a particular social group (Clement et al., 2015).

In a systematic review of empirical studies investigating stigma associated with mental health help-seeking behaviors and attitudes, stigma was found to be a commonly reported barrier to help-seeking (Clement et al., 2015). Stigma was observed to have a consistent moderate negative association with psychological help-seeking behaviors and attitudes across studies (Clement et al., 2015). Results also indicated that the effect of stigma on mental health help seeking is not equal across groups. In fact, Clement et al. (2015) found that ethnic minorities, youth, men, military members, and health professionals were disproportionately deterred from seeking psychological help due to stigma. For example, those from Asian, Arab, and African American minority groups were more affected by stigma than others. For Arab students, the median negative association between stigma and help-seeking was large across studies (Clement et al., 2015).

Discrimination and stigma do not operate in an isolated manner but rather as an intersecting system in which marginalized identities play a simultaneous and often compounding role (Ciftci et al., 2013; Fiske, 2014). The present study seeks to examine the relationships between perceived discrimination and self-stigma due to this interplay. For individuals with multiple marginalized identities, the experience of stigma may be magnified or more complex (Ciftci et. al., 2013). An intersectional lens should be used to conceptualize attitudes and 


\section{BARRIERS AND FACILITATORS OF SEEKING PSYCHOLOGICAL HELP}

behaviors related to seeking mental health treatment given the complex relationships at play between different marginalized identities such as race, gender, sexual orientation, nationality, class, and disability (Ciftci et al., 2013). For example, individuals from under-represented and minority populations often face "double stigma", experiencing prejudice and discrimination related to both their racial identity and mental illness (Gary, 2005). In addition, when conceptualizing stigma from an intersectional framework, it is understood that the effect of stigma differs by both degree (amount) and kind (type and effect) based on one's identities, such that the amount and negative effects of stigma are often greater for those with higher numbers of marginalized identities (Ciftci et al., 2013). Demographic differences such as class, educational attainment, religious beliefs, and gender have all been found to impact the way members of ethnic and cultural minority groups experience and perpetrate mental illness stigma in the U.S. (Ciftci et al., 2013).

Types of stigma. Four types of stigma (public, structural, self, and by association) have been identified in the literature (Bos et al., 2013). Public stigma is based on cognitive representations and encompasses the social, cognitive, affective, and behavioral reactions people have to one another (Bos et al., 2013). It is an external form of stigma held by a society deeming certain individuals or traits as socially undesirable or unacceptable based on stereotypes and prejudice (Ciftci et al., 2013; Vogel et al., 2017). Public stigma often results in discrimination such as abuse, segregation, or preventing fair access to employment, educational opportunities, health care, and housing (Ciftci et al., 2013). MUS often face public stigma based on their religion. For example, when an individual sees men wearing turbans, assumes they are terrorists, and shouts at them, they are experiencing the result of public stigma (Ciftci et al., 2013). Mental health concerns and psychological treatment also carry public stigma, both for the affected 


\section{BARRIERS AND FACILITATORS OF SEEKING PSYCHOLOGICAL HELP}

individual and their family members (Amri \& Bemak, 2013). Often the public misunderstands the progression and effect of mental illness and stigmatizes those with mental health concerns as “crazy" or "dangerous". Blame, incompetence, and undesirability are stereotypes that are frequently applied to those with mental illness (Corrigan, Larson, \& Rusch, 2009; Vogel et al., 2017). Public stigma related to seeking psychological help occurs cross-culturally and has been cited in the literature arising in Australia, Canada, China, Hong Kong, Portugal, Romania, Taiwan, Turkey, the United Arab Emirates, and the United States (Vogel et al., 2017).

Linked with cultural and religious values, public stigma regarding mental illness exists in the Muslim community and is often attributed to a lack of faith, possession by a spirit, bad karma, or the evil eye (Ciftci et al., 2013). These cultural and religious beliefs can contribute to public stigma on behalf of the Muslim religious community and a fear that seeking psychological help could lead to individual and family shame, the perception of being weak, and a loss of face (Aloud \& Rather, 2009; Amri \& Bemak, 2013; Youssef \& Deane, 2006). Some Arab Muslim individuals believe it is shameful or inappropriate to seek help for psychological or social problems from those outside the religious or cultural community and report feelings of guilt for doing so (Aloud \& Rather, 2009; Yousef \& Deane, 2006). Al-Adawi et al. (2002) conducted a study using a sample of medical students and members of the general public in Oman to assess perceptions of and attitudes towards mental illness. They found that participants rejected the idea that a genetic factor could be the cause of mental illness, instead emphasizing the role of spirits in causing mental illness. In addition, participants endorsed beliefs that people with mental illness tended to have peculiar and stereotypical appearances and should be cared for in psychiatric facilities located away from the general public (Al-Adawi et al., 2002). Likewise, a study assessing attitudes toward mental health issues among Pakistani individuals in the United 


\section{BARRIERS AND FACILITATORS OF SEEKING PSYCHOLOGICAL HELP}

Kingdom, demonstrated evidence of public stigma toward mental health in the Muslim community as participants reported that they would not consider marriage with someone with mental illness (Tabassum, Macaskill, \& Ahmad, 2000). Furthermore, half of the participants reported they would not even socialize with a person with mental illness (Tabassum et al., 2000). Public stigma is thought to play a role in enforcing self-stigma, structural stigma, and stigma by association (Bos et al., 2013).

Stigma by association occurs when stigmatizing social and psychological reactions are transferred from a person with a stigmatized quality to those closely associated with him or her (Bos et al., 2013). Arab individuals may deny mental health concerns for fear that acknowledging these problems could bring shame on their family members or influence their standing within the community (Aloud \& Rather, 2009; Corrigan \& Watson, 2002). For example, Arabs living in Australia reported fear that mental illness could negatively influence their marriage prospects (Youssef \& Deane, 2006). Structural stigma occurs when a stigmatized status is legitimized and perpetuated through a society's institutions and ideological systems (Bos et al., 2013). For example, when an institution such as a newspaper disperses stigmatizing messages about mental illness or Islam, MUS experience structural stigma (Corrigan et al., 2005).

Self-stigma or internalized stigma, the construct of interest in the present study, is often a result of public stigma and occurs when an individual internalizes external prejudice directed at their group (Ciftci et al., 2013; Clement et al., 2015; Vogel et al., 2017). Self-stigma is the result of the social and psychological impact of facing discrimination and marginalization from society. It is comprised of apprehension of being stigmatized as well as the internalization of the negative beliefs and feelings others have associated with the denigrated identity (Bos et al., 2013). Self- 


\section{BARRIERS AND FACILITATORS OF SEEKING PSYCHOLOGICAL HELP}

stigma is a process that includes awareness, agreement, application, and harm (Ciftci et al., 2003). First, one must be aware of the stereotype related to a given identity (e.g., race, religion, gender, nationality) they hold. Following awareness, self-stigma builds when the individual agrees with the negative stereotype regarding their identity group (e.g., that Muslims are extremists) and applies it to themselves (e.g., my religion is extreme). Finally, self-stigma results in harm to the individual as it diminishes their self-esteem and self-efficacy due to the internalized negative beliefs (Ciftci et al., 2013). Essentially, self-stigma is a harmful internal belief that one is unacceptable or undesirable due to a given personal trait deemed as negative by society (Vogel et al., 2017).

One pernicious effect of self-stigma is that it often results in foreclosure of important life goals as the stigmatized individual self-selects out of pursuits that society views as negative or unattainable for that individual due to a particular unfavorable identity (Ciftci et al., 2013). For example, self-stigma has been found to undermine mental health treatment (Clement et al., 2015; Corrigan et al., 2009). Self-stigma for seeking psychological help, the outcome variable of this study, occurs when an individual believes that he or she would be undesirable or socially unacceptable for seeking mental health services (Vogel et al., 2017). For example, in a sample of Arabic-speaking individuals living in Australia, participants noted strong cultural prohibitions on revealing personal or family problems to outsiders and doing so was connected to significant shame and stigma (Youssef \& Dean, 2006). These findings demonstrate the interplay between stigma and culture as cultural beliefs and constructs often play a role in determining what is stigmatized. It is likely that religiosity and acculturation play a role in self-stigma for seeking psychological help because both religious factors and cultural norms shape understanding about 


\section{BARRIERS AND FACILITATORS OF SEEKING PSYCHOLOGICAL HELP}

psychological problems and acceptable ways to address them (Clement et al., 2015; Cifti et al., 2013).

Self-stigma is thought to play a role in treatment seeking and utilization for those with mental health concerns. Individuals with mental health concerns often experience dissonance between their preferred self-identity and frequently held stereotypes about mental illness. The personal application of these stereotypes and resulting negative consequences (such as shame, embarrassment, rejection, and discrimination) are anticipated by the individual with mental health concerns. To avoid the negative consequences, individuals often hide their symptoms and avoid help-seeking (Clement et al., 2015).

Modified labeling theory and the "why try" effect are used to understand the negative internal results of stigma in relation to mental health treatment. These models propose that people who experience self-stigma about mental illness undergo negative effects to their selfesteem and self-efficacy. This occurs when they are 1) aware of the stigma attached to mental illness, 2) agree that the stereotype is true, and 3) apply the stereotype to themselves (Corrigan et al., 2009). When these conditions are present, the result is often that an individual fails to pursue important life goals, including finding stable housing, employment, education, or even seeking out mental health services. In effect asking "why try?" in response to public stigma and subsequent self-stigma with internalized negative beliefs such as 'a person like me is not capable of a goal like that' (Corrigan et al., 2009). In a systematic review of the effects of stigma on mental health, a consistent small negative effect was found between self-stigma and helpseeking. They also found a consistent small negative effect for treatment stigma, the fear of seeking treatment due to negative perceptions on mental illness (Clement et al., 2015). 


\section{BARRIERS AND FACILITATORS OF SEEKING PSYCHOLOGICAL HELP}

Self-stigma has been found to fully mediate the relationship between public stigma and attitudes toward mental health services in U.S. samples (Vogel, Wade, \& Hackler, 2007). Associations between public stigma, self-stigma, and negative attitudes toward psychological services have also been demonstrated for career counseling and group counseling (Vogel et al., 2017). Some research has demonstrated differences in patterns of stigma of mental illness based on sex, gender, racial identity, religious beliefs, and culture (Ciftci et al., 2013). MUS with mental health concerns, frequently also experience racism, sexism, and Islamophobia, which intersect to magnify the oppression they face and can result in internalized self-stigma. It is possible that higher religious practice is associated with a greater likelihood to experience discrimination, less acculturation, and greater self-stigma, the focus of the present study.

Some scholars argue that self-stigma is the primary predictor of attitudes toward seeking mental health help, although slight cultural differences do exist (Vogel et al., 2017). Self-stigma should be considered with the role of culture in mind. Self-stigma of seeking psychological help has been documented in countries including England, Greece, Israel, Turkey, Taiwan, the United States, Australia, Brazil, Canada, Hong Kong, Portugal, Romania, and the United Arab Emirates (Vogel et al., 2017). Using structural equation modeling, Vogel et al. (2017) found that selfstigma mediated the relationship between public stigma and attitudes towards seeking psychological help among college students in 10 different countries, including Australia, Brazil,

Canada, Hong Kong, Portugal, Romania, Taiwan, Turkey, United Arab Emirates, and the United States. Weaker associations between public and self-stigma were found in Brazil, Portugal, Romania, Taiwan, and Turkey as compared to the other countries included in the study. Similarly, the associations between self-stigma for seeking psychological help and attitudes toward seeking psychological help were weaker for samples from Hong Kong, Turkey, and the 


\section{BARRIERS AND FACILITATORS OF SEEKING PSYCHOLOGICAL HELP}

UAE than the average for the other countries or regions included in the study (Vogel et al., 2017). The authors stated that these differences could have been influenced by differences in cultural values or perception of those who seek help (Vogel et al., 2017). In addition, they noted that Hong Kong, Turkey, and the UAE have all experienced rapid social, cultural, and economic shifts leading to a blend of Western and non-Western cultural ideas, particularly among younger generations (Vogel et al., 2017). For this reason, the impact of acculturation and religiosity (an important cultural component for predominately Muslim countries such as Turkey and the UAE) should be considered when exploring self-stigma of seeking psychological help. Previous research suggest that more acculturation to Western culture is associated with less stigma for seeking psychological help and this finding should be investigated further in future studies.

Ciftci et al. (2013) noted the need for additional quantitative research regarding stigma within the Muslim community in the United States. Specifically, they noted the need for stigma research that is sensitive to the involvement of families and religious leaders, intersectional stigma (addressing stigma due to race, class, gender, and Islamophobia), and culturally specific explanatory models for mental illness and treatment. MUS may fear seeking out mental health services due to being stigmatized within their communities or due to fear of facing discrimination within the mental health settings (Amri \& Bemak, 2013). This may be exacerbated by level of acculturation because formal mental health treatment originated in Western societies and could be less familiar in the cultural contexts experienced by some Muslims in the U.S. (Ciftci et al., 2013). Enhanced understanding of culturally bound barriers and facilitators of mental health treatment seeking could improve access to non-stigmatizing forms of care such as practitioners' use of terms that reflect clients' understanding of their problems (Clement et al., 2015). 


\section{BARRIERS AND FACILITATORS OF SEEKING PSYCHOLOGICAL HELP}

\section{Summary}

Due to increased social, cultural, and political struggles for MUS, affordable, accessible, and culturally appropriate mental health care is important for these individuals (Aloud \& Rathur, 2009). Yet, mental health services are underutilized by this group (Aloud \& Rather, 2009;

Martin, 2015). Research examining the unique mental health needs and behaviors of MUS has been sparse overall (Abuelezam et al., 2017; Abu Raiya et al., 2007). The present study seeks to understand barriers and facilitators of seeking formal mental health services for MUS by examining aspects of Aloud and Rather's (2009) HSPAM model. The HSPAM model proposes that Islamic and ethnic cultural factors influence mental health help seeking beliefs and practices for MUS. In addition, the model asserts that perceived societal stigma towards mental illness, negative attitudes towards provider influence, and availability of alternative resources such as family and friends influence Muslims' decision to seek help (Aloud \& Rather, 2009). Specific service selection is then thought to be influenced by acculturation level, awareness of formal services, use of informal resources, and economic and institutional barriers (Aloud \& Rather, 2009). While societal stigma is thought to influence decision to seek help, it is likely that selfstigma for seeking psychological help also plays a role. Self-stigma for seeking psychological help occurs as result of internalizing societal stigma and discrimination (Ciftci et al., 2013; Vogel et al., 2017). Further research of this conceptual model should be conducted to better understand and extend these pathways.

MUS, especially those with mental health concerns, hold a number of stigmatized identities, often including their race and ethnicity, religion, and mental health status (Ciftci et al., 2013). Furthermore, mental health attitudes and service selection are impacted by Islamic beliefs and practices and acculturation (Abu Raiya et al., 2007; Ciftci et al., 2013). This study examines 


\section{BARRIERS AND FACILITATORS OF SEEKING PSYCHOLOGICAL HELP}

the nature of the relationship between Islamic beliefs and practices and self-stigma of seeking psychological help, with acculturation and perceived religious and ethnic discrimination serving as potential mediator variables in that relationship.

\section{Research Questions}

The research questions of the current study are:

1) Is there a relationship between Islamic beliefs and practices and self-stigma of seeking psychological help in a MUS sample?

2) Does acculturation mediate the relationship between Islamic beliefs and practices and self-stigma of seeking psychological help in a MUS sample?

3) Does perceived religious discrimination mediate the relationship between Islamic beliefs and practices and self-stigma of seeking psychological help in a MUS sample?

4) Does perceived ethnic discrimination mediate the relationship between Islamic beliefs and practices and self-stigma of seeking psychological help in a MUS sample?

\section{Hypotheses}

Hypothesis 1. Higher levels of Islamic practices and beliefs as measured by the Short Muslim Practice and Belief Scale (S-MPBS; AlMarri, Oei, \& Al-Adawi, 2009) will be positively related to self-stigma of seeking psychological help as measured by the Self-Stigma of Seeking Help Scale (SSOSH; Vogel et al., 2006) in a MUS sample.

Hypothesis 2. The association between Islamic practices and beliefs and self-stigma of seeking psychological help will be partially mediated by acculturation to majority American culture as measured by the Attraction to American Culture Subscale (AAmC) of the Acculturation Rating Scale for Arabic Americans-II (ARSAA-II; Jadalla \& Lee, 2015) in a MUS sample. 
BARRIERS AND FACILITATORS OF SEEKING PSYCHOLOGICAL HELP

Hypothesis 3. The association between Islamic practices and beliefs and self-stigma of seeking psychological help will be partially mediated by perceived ethnic discrimination as measured by the Brief Perceived Ethnic Discrimination Questionnaire - Community Version Lifetime Exposure Scale (PEDQ-CV; Brondolo et al., 2005) in a MUS sample.

Hypothesis 4. The association between Islamic practices and beliefs and self-stigma of seeking psychological help will be partially mediated by perceived religious discrimination as measured by the Religious Discrimination Scale (RDS; Kawika Allen, Wang, Richards, Ming, \& Suh, 2018) in a MUS sample. 
BARRIERS AND FACILITATORS OF SEEKING PSYCHOLOGICAL HELP

\section{CHAPTER 2: METHOD}

\section{Participants}

The qualification criteria for this study were that participants must be at least 18 years of age, identify as Muslim, read English fluently, and have lived in the U.S. for three or more months. These criteria were chosen because they allowed for broad recruitment of Muslims in the U.S. (MUS) consistent with the diverse and multidimensional nature of this group. Participants were recruited through convenience sampling and social network sampling. Recruitment was conducted through contact with mosques, university Muslim Student Associations, social media groups, relevant list-serves, and professional contacts relevant to this population. Emails and social media posts such as on Facebook were sent to members and pages for those listed above. Message content included a short description of the study, indication of Institutional Review Board (IRB) approval, eligibility requirements, information regarding participation compensation, and a request to distribute the survey to members (see Appendix G). Participants completing the full survey were eligible to enter to win one of 10 available $\$ 25$ Amazon gift cards.

The final sample size consisted of 202 participants for the present study. Kline (2005) and Kenny (2015) noted that a sample size of 200 is generally acceptable for structural equation modeling, the analysis to be used in the present study. See Table 1 for participant demographic information.

\section{Measures}

Each of the measures chosen for this study were selected based on their psychometric properties and assessment of the constructs in question. Given the relatively sparse research related to Muslims living in the United States (Amer, \& Bagasra, 2013; Ciftci et al., 2013) and 


\section{BARRIERS AND FACILITATORS OF SEEKING PSYCHOLOGICAL HELP}

the paucity of culturally valid tools for this population (Amer, \& Bagasra, 2013) the scales selected represent the best available at the time.

Self-Stigma of Seeking Help Scale (SSOSH; Vogel et al., 2006). The SSOSH is a 10item self-report Likert scale that was used in the present study as an outcome variable to measure participant level of self-stigma related to seeking psychological help (see Appendix A). Response anchors on the survey ranged from 1 (strongly disagree) to 5 (strongly agree) with a neutral middle anchor of 3 (agree and disagree equally). Five items were reverse scored. A total score is calculated by adding together the scores of each item. Higher scores indicate higher levels of self-stigma. Items include statements such as "Seeking psychological help would make me feel less intelligent," and "If I went to a therapist I would be less satisfied with myself."

The scale was validated on a sample of 470 U.S. college students and demonstrated strong internal consistency reliability (Cronbach's alpha $=.89)$ (Vogel et al., 2006). Evidence of construct validity was demonstrated as the SSOSH was positively associated with measures of public stigma, such as the Social Stigma for Seeking Psychological Help Scale (SSRPH; Komiya, Good, \& Sherrod, 2000), and with anticipated risks of emotional problems disclosure expectations, such as the Disclosure Expectations Anticipated Risks Scale (DES Anticipated Risks; Vogel \& Wester, 2003). Likewise, evidence of criterion validity was demonstrated as the SSOSH was negatively associated with measures of anticipated benefits of disclosure expectations Disclosure Expectations Anticipated Benefits Scale (DES Anticipated Benefits; Vogel \& Wester, 2003), intentions to seek counseling (Intentions to Seek Counseling Inventory;

ISCI: Cash, Begley, McCown, \& Weise, 1975), and positive attitudes towards seeking psychological help (Attitudes Toward Seeking Professional Psychological Help; ATSPPHS; 


\section{BARRIERS AND FACILITATORS OF SEEKING PSYCHOLOGICAL HELP}

Fischer \& Farina, 1995) (Vogel et al., 2006). Additionally, the SSOSH revealed good test-retest reliability over a 2-month period, although values were not provided (Vogel et al., 2006).

The SSOSH has been previously translated and used in international populations including two predominantly Muslim countries, Turkey and the United Arab Emirates (UAE) (Vogel et al., 2013; Vogel et al., 2017). In the Turkish population, the SSOSH demonstrated adequate internal consistency reliability (Cronbach's alpha $=.82)$ and through configural invariance analysis the authors demonstrated that the single factor structure held for the Turkish sample. Vogel et al. (2017) used back-translation procedures to translate the SSOSH scale into Arabic using two bilingual independent translators. After translation, an expert faculty member from the region reviewed the translations and revised wording when necessary. The scale was used to measure self-stigma in a sample of 417 UAE college students and again demonstrated adequate reliability (Cronbach's alpha $=.77)$. In the present study, the scale reliability was good $($ Cronbach's alpha $=.85)$.

\section{Acculturation Rating Scale for Arabic Americans-II, Attraction to Arabic Culture}

Subscale (ARSAA-II; AAmC; Jadalla \& Lee, 2015). The ARSAA-II has two subscales, Attraction to American Culture (AAmC) and Attraction to Arabic Culture (AArC). For the current study, only the Attraction to American Culture (AAmC, 13 items) subscale of the ARSAA-IIE (English version) was used as a predictor variable to measure participant acculturation levels (see Appendix B). The AArC subscale was not used in the present study because the variable of interest is acculturation to majority American culture. Additionally, the AArC subscale would not be appropriate for use due to the diversity of ethnic heritage of Muslims living in the U.S., many of whom are not connected to Arab heritage. Finally, 


\section{BARRIERS AND FACILITATORS OF SEEKING PSYCHOLOGICAL HELP}

eliminating the AArC subscale decreased survey length and facilitated greater likelihood of participant response completion.

The AAmC is a self-report Likert scale and includes items such as "My friends are now of White origin." Items are rated on a 5-point scale with anchors of 1 (Not like me) to 5 (A lot like me). Higher scores indicate greater attraction and acculturation to White American majority culture (Jadalla \& Lee, 2015). None of the items are reverse scored. A mean score is calculated for the AAmC subscale by dividing the total by the number of items for that scale (13 items). In the present study, the internal consistency of the AAmC items was adequate (Cronbach's alpha $=$ $.73)$.

The ARSAA-IIE was based off the Acculturation Rating Scale of Mexican-Americans II (ARSMA-II; Cuellar, Arnold, \& Maldonado, 1995) which is comprised of two cultural orientation subscales, the Anglo Orientation Subscale (AOS) and Mexican Orientation Subscale (MOS). Each of these cultural orientation subscales demonstrated good internal reliability (AOS Cronbach's alpha $=.86$; MOS Cronbach's alpha $=.88$ ). The ARSMA-II demonstrated strong evidence of construct validity in the validation sample of 379 Mexican Americans from the first through fifth generations living in the United States, although no values were given (Cuellar et al., 1995).

Barry's (2005) multidimensional framework of acculturation served as the theoretical framework for the original ARSMA-II as well as the ARSAA-IIE adaptation. Barry's (2005) framework includes four models of acculturation which include: assimilation, integration, separation, and marginalization. The ARSAA-II scale thereby captures the multidimensional nature of acculturation by focusing on various aspects of the process including language, ethnic identification, and cultural practices. 


\section{BARRIERS AND FACILITATORS OF SEEKING PSYCHOLOGICAL HELP}

The AAmC acculturation subscale used in the present study was translated and adapted for use in this population by changing words that referred to Mexican American culture to those that reflected Arab and majority White American culture. In addition, the authors underwent an in-depth process following the steps of Brislin (1986) and Jones, Lee, Phillips, Zhang, and Jaceldo (2001) to translate the ARSMA-II into formal Arabic (Logha Fos'ha) using back translation procedures and blind-evaluation by bilingual professionals (Jadalla \& Lee, 2015).

Following translation, pilot-testing of the scale was conducted with Arab individuals who were fluent Arabic speakers and reported that scales did not render any linguistic difficulties or dialectical bias because formal Arabic had been used, demonstrating the linguistic and cultural validity of the scale (Jadalla \& Lee, 2015). In the pilot sample $(n=30)$, the ARSAA-IIE $($ Cronbach's alpha $=.85)$ and the ARSSAA-IIA $($ Cronbach's Alpha $=.73)$ demonstrated adequate internal consistency reliability.

In the full study, 297 Arab American participants were recruited to complete either the ARSAA-IIA (41\%) or the ARSAA-IIE (59\%). Participants were predominantly Muslim (67\%), female $(56 \%)$, foreign-born (85\%) with an average age of 38.6 and average income of $\$ 66,000$ per year. Factor analysis was used to determine whether the previous factor structure for the Mexican American scale fit for this new population. The loadings diverged slightly from that described by Cuellar et al., (1995) such that fifteen items each loaded on the Orientation to American Culture subscale, rather than the thirteen original items. In addition, two items on the Orientation to American Culture subscale were weakly correlated with the other items on the scale for both language groups so they were dropped from the subscale. The final scale was comprised of two factors, renamed as Attraction to American Culture (AAmC) and Attraction to Arabic Culture (AArC), which were similar to the factors to those of the ARSMA-II, 


\section{BARRIERS AND FACILITATORS OF SEEKING PSYCHOLOGICAL HELP}

demonstrating validity for each scale (Jadalla \& Lee, 2015). The internal consistency reliability for the final AAmC (Cronbach's alpha $=.89)$ and AArC (Cronbach's alpha $=.85)$ were good.

There were some differences of note between those who filled out the English or Arabic versions of the scale. Participants who filled out the scale in English demonstrated statistically significantly higher scores of AAmC $(M=3.5, S D=.62)$ compared to those who filled out the scale in Arabic $(M=2.44, S D=.67)$ (Jadalla \& Lee, 2015). Likewise, English respondents had statistically significantly lower scores on the AArC subscale $(M=3.93, S D=.61)$ than their Arabic speaking counter parts $(M=4.44, S D=.46)$. On average, English respondents had a statistically significant longer length of residence in the U.S. $(M=19.2$ years, $S D=9.8)$ compared to the Arabic respondents $(M=14.9$ years, $S D=10.6)$. Religion also differentiated participant scores, such that scores of AAmC were higher for Christian participants $(M=3.30$, $S D=.78)$ than their Muslim counter-parts $(M=2.97, S D=.86), t(202)=-3.31, p=.001$. Similarly, Muslim participant scores $(M=4.18, S D=.51)$ were higher than their Christian counterparts $(M=3.99, S D=.58), t(165)=2.63, p<.01$ on the AArC. Finally, higher levels of education were correlated with higher levels of AAmC $(r=.331, p<.01)$, whereas higher levels of education were negatively correlated with $\operatorname{AArC}(r=-.136, p<.01)$. These significant associations and differences demonstrate the validity of the ARSAA-II to measure acculturation due to the fact that language acquisition and length of residence are often used as proxy measures to assess acculturation (Jadalla \& Lee, 2015). Furthermore, due to these significant associations, the length of time living in the United States, and educational level were treated as controls in the present study.

Short Muslim Practice and Belief Scale (S-MPBS; AlMarri et al., 2009). The S-

MPBS was used as a predictor variable to measure participants' fundamental principles of 


\section{BARRIERS AND FACILITATORS OF SEEKING PSYCHOLOGICAL HELP}

religious practice as well as their general religious beliefs as pertaining to Islam (see Appendix C). This 9-item Likert scale measure was designed to differentiate between participants with more relaxed versus dogmatic Islamic beliefs. Four items are related to principles of religious practice based on the pillars of Islam and include statements such as "I pray five times a day." Islamic practice items were rated on a 5-point scale ranging from 1 (I never do this) to 5 (I always do this). Five items relate to general religious belief and flexibility and include statements such as "Religion should govern all my actions." Religious belief items were rated on a 5-point scale ranging from 1 (strongly disagree) to 5 (strongly agree). Subscale scores are totaled and added together. Higher scores indicate a higher commitment to the acts and beliefs associated with Islam (AlMarri et al., 2009). In the present study, the internal consistency of the S-MPBS items was good (Cronbach’s alpha $=.89)$.

The S-MPBS was validated using a sample of 914 Arab and Asian Muslim participants including Emirati or Arab nationals living in the United Arab Emirates $(n=433)$, Omani nationals $(n=178)$, Indonesian nationals $(n=152)$, and Malaysian nationals $(n=151)$. The measure was translated from English to Arabic by two separate translators and then backtranslated in order to confirm clarity of meaning (AlMarri et al., 2009). Either an English or Arabic version can be used. Responses were randomly split into two groups. The first group ( $n$ $=415)$ was used for exploratory factor analysis and confirmatory factor analysis (CFA). A twofactor solution was supported through exploratory factor analysis after dropping two of the original 13 items. The initial confirmatory factor analysis further validated the two-factor structure after dropping a final two items due to low loadings. The second group $(n=431)$ was used to further validate the scale with a second confirmatory factor analysis and again demonstrated good fit for the two-factor model (AlMarri et al., 2009). 


\section{BARRIERS AND FACILITATORS OF SEEKING PSYCHOLOGICAL HELP}

Next, the total sample was divided by ethnicity with a total of 561 Arab participants and 285 Asian participants for multigroup CFA analysis. The modified two factor model demonstrated an acceptable fit for both groups with the specified model being consistent across samples, although differing slightly at the individual item level (AlMarri et al., 2009). The internal consistency reliability was good for the overall scale (Cronbach's alpha $=.83$ ), religious practice subscale (Cronbach's alpha $=.82)$, and the religious beliefs subscale (Cronbach's alpha $=.83$ ). Islam strictly forbids drinking alcohol. Using discriminant function analysis, this scale was able to discriminate between lifetime alcohol abstainers and those who drank, further demonstrating the validity of the scale. Neither, test-retest reliability, nor convergent validity studies were reported for this measure (AlMarri et al., 2009).

\section{Brief Perceived Ethnic Discrimination Questionnaire - Community Version}

\section{Lifetime Exposure Scale (PEDQ-CV; Brondolo et al., 2005). The Brief PEDQ-CV Lifetime}

Exposure Scale was used as a predictor variable to measure participants' perceived experiences of different types of interpersonal maltreatment based on ethnicity or race (see Appendix D). This scale was created based on the PEDQ-Revised B, a scale originally developed to measure college students' experience of racism or ethnic discrimination across ethnic groups (Contrada et al., 2001). The scale was then amended to be applicable to community adults. After making initial revisions, focus groups and individual interviews were conducted with community members from different racial and ethnic backgrounds. The final version of the scale was then piloted in a sample of college students as well as a sample of community members (Kwok et al., 2011). The full PEDQ-CV is a 62-item measure; however, the 17-item Brief Lifetime Exposure subscale was used in this study. In the present study, the internal consistency of the Brief PEDQ-CV items was good (Cronbach's alpha $=.92)$. 


\section{BARRIERS AND FACILITATORS OF SEEKING PSYCHOLOGICAL HELP}

The Brief PEDQ-CV Lifetime Exposure Scale has been used and validated in Black, Latino, and Asian (including Pakistani and Bangladeshi) populations (Brondolo et al., 2005; Kwok et al., 2005). It produces a total score and also four subscale scores that measure social exclusion (4 items), stigmatization (5 items), discrimination at work or school (4 items), and threat/aggression (4 items) (Brondolo et al., 2005). Participants are instructed to rate how often they had been affected by listed experiences due to their race or ethnicity during their lifetime. Each subscale is made up of four items which include "Made you feel like an outsider because of appearance" (exclusion), "hinted you must be lazy" (stigmatization), "treated unfairly by teachers" (discrimination at work/school), and "actually hurt you" (threat/aggression). An additional item assesses exposure to police brutality as that is often a source of ethnicity-related stress for many people of color. Each item is rated on a 5-point Likert scale with anchors of 1 (never happened) to 5 (happened very often) (Brondolo et al., 2005).

The Brief PEDQ-CV Lifetime Exposure Scale was originally validated with a male and female sample of 128 college students and 212 community members identifying as Black or Latino. The internal consistency reliability was good for the overall scale (Cronbach's alpha $=$ $.87)$, exclusion/rejection subscale (Cronbach's alpha $=.70)$, stigmatization/devaluation subscale $($ Cronbach's alpha $=.74)$, discrimination at work/school subscale $($ Cronbach's alpha $=.69)$, and the threat aggression subscale (Cronbach's alpha $=.80$ ) (Bronodolo et al., 2005). Convergent validity was demonstrated as the Brief PEDQ-CV Lifetime Exposure Scale correlated with the Perceived Racism Scale (PRS; McNeilly et al., 1996), Black $(r=.61, p<.001)$ and Latino $(r=$ $.57, p<.001)$ versions. Concurrent validity was demonstrated as scale scores were positively associated with both threat $(r=.43, p<.0001)$ and harm $(r=.46, p<.0001)$ appraisals. Similarly, scale scores were not significantly associated with primary appraisals of challenge $(r=$ 


\section{BARRIERS AND FACILITATORS OF SEEKING PSYCHOLOGICAL HELP}

$.09, p>.22)$ and were weakly correlated with perceptions of benefit $(r=.18, p<.01)$, demonstrating evidence of discriminant validity. Construct validity was further demonstrated as scale scores remained significantly correlated with threat $(r=.27, p<.02)$ and harm $(r=.37, p<$ .001) appraisals after controlling for trait scores of anxiety, defensiveness, hostile attributions, and cynicism. This data suggests that the Brief PEDQ-CV Lifetime Exposure Scale demonstrates good psychometric properties.

Religious Discrimination Scale (RDS; Kawika Allen et al., 2018). The RDS is an 11item self-report Likert scale that was used as a predictor variable to measure participants' experience of perceived religious discrimination (see Appendix E). The RDS is comprised of three subscales including Perceived Prejudice (five items), Closet Symptoms (three items), and Negative Labels (3 items). Items include statements such as "I sense hostility from others because of my religious affiliation" (Perceived Prejudice), "I do not feel free to express who I am religiously" (Closet Symptoms), and "I have heard people make unfriendly remarks about my religion" (Negative Labels). Each item is rated on a 5-point Likert scale with anchors of 1 (never) to 5 (always) (Kawika Allen et al., 2018). In the present study, the internal consistency for the RDS items was good (Cronbach's alpha $=.91)$.

Researchers developed a list of 22 items for the RDS by editing items found on discrimination scales used for other minority groups including racial and sexual minorities (Kawika Allen et al., 2018). An exploratory factor analysis (EFA) was then conducted using a sample of 270 participants who primarily identified as Latter-day Saints. Results suggested a three-factor solution. Items that had factor loadings greater than .40 , cross loadings of less than .30, and consistent item content were retained (Kawika Allen et al., 2018). A second EFA was conducted with the selected 11 items and the three-factor solution was shown to account for 


\section{BARRIERS AND FACILITATORS OF SEEKING PSYCHOLOGICAL HELP}

$65.17 \%$ of the total variance explained (Kawika Allen et al., 2018). Factor loadings for the Perceived Prejudice subscale items were .62 to .83 and accounted for $47.3 \%$ of the variance before rotation. Factor loadings for Closet Symptoms items were between .62 and .89 and accounted for $17 \%$ of the variance prior to rotation. Factor loadings for the Negative Labels items were between .64 and .94 and accounted for $9.9 \%$ of the total variance before rotation (Kawika Allen et al., 2018). A confirmatory factor analysis (CFA), conducted using an additional sample of 274 individuals to cross-validate the RDS, yielded adequate fit statistics for the three-factor model $[\mathrm{CFI}=.948, \mathrm{SRMR}=.059, \mathrm{RMSEA}=.068$ (90\% C.I. .049-.086) $]$ (Kawika Allen et al., 2018). The authors also conducted a multiple-group CFA to test measurement invariance between male and female participants and found structural invariance between genders, suggesting the RDS can be appropriately used with both males and females (Kawika Allen et al., 2018). The mean scores of the RDS subscales were lower for Perceived Prejudice $(M=1.97)$ and Closet Symptoms $(M=1.94)$ than for Negative Labels $(M=3.36)$ in the scale development sample (Kawika Allen et al., 2018).

Although this scale was originally developed using a population that predominately identified as Latter-day Saints, the RDS is one of the only scales developed to date that reliability and validly measures religious discrimination (Kawika Allen et al., 2018). Religious discrimination is an often-reported phenomenon but few attempts have been made to empirically measure it, thus, although not yet validated using an Islamic sample, the RDS is at present the best scale available to measure this construct.

Generalizability Theory Study (G-study) was used to determine scale reliability as it has been used for its strength to distinguish multiple sources of errors, thus providing reliability of measurement that can be generalized to other situations (Kawika Allen et al., 2018). The authors 


\section{BARRIERS AND FACILITATORS OF SEEKING PSYCHOLOGICAL HELP}

found adequate generalizability coefficients for each subscale. An overall scale generalizability coefficient was not provided because the authors suggested not using the composite RDS total score due to poor single-factor model fit (Kawika Allen et al., 2018). The Perceived Prejudice subscale score had generalizability coefficients of .89 and .85 in sample one and two, respectively (Kawika Allen et al., 2018). The generalizability coefficients were .85 in sample one and .78 in sample two for the Closet Symptoms subscale. The Negative Labels subscale had generalizability coefficients of .82 and .79 in sample one and two, respectively (Kawika Allen et al., 2018). Thus, evidence of reliability and validity was demonstrated for this scale due to its strong and clear factor structure and good internal consistency reliability (Kawika Allen et al., 2018).

Demographic questionnaire. Using a 14-item measure, participant demographic information including age, race, ethnicity, gender, socioeconomic status, length of time living in the U.S., nationality, region of origin, preference for and previous experience addressing mental health concerns, marital status, religion, generational status, primary language, and educational level was collected though a forced-choice questionnaire (see Appendix F).

\section{Procedure}

Approval for this study was obtained through the West Virginia University Institutional Review Board. Upon approval, participant recruitment and data collection began. Participants were recruited through convenience sampling and social network sampling. The final analytical sample consisted of $n=202$ participants. Recruitment emails were sent to the Muslim Student Association leadership teams for the flagship universities of all 50 states in the USA. Recruitment emails were also sent to the training directors of psychology departments at universities in the USA asking them to distribute the survey to their students. The cover letter 


\section{BARRIERS AND FACILITATORS OF SEEKING PSYCHOLOGICAL HELP}

and survey were distributed on social media and the post settings were set to encourage the post to be shared widely to reach a greater number of potential participants. The recruitment letter and survey were also sent to Muslim religious leaders and leaders of Islamic networks (i.e., the Islamic Network Group) in the USA for distribution among members. The cover letter and survey were sent to contacts made at the 2018 Muslim Mental Health Conference in Washington, D.C. which were then disseminated to additional networks. The recruitment letter and survey were also distributed on the list-serves for the American Psychological Association (APA) Division 17 Society of Counseling Psychology, APA Division 45 Society for the Psychological Study of Culture, Race, and Ethnicity, and APA Division 36 Society for the Psychology of Religion and Spirituality. The decision was made to recruit via APA list serves only in the final months of data collection when all other recruitment means were exhausted, and a larger sample was needed. In the initial data collection phase, it was determined that the survey had been fraudulently filled out by robots in an effort to obtain the gift cards which had originally been offered to the first 200 participants to complete the survey at a rate of $\$ 5.00$ each. After working with WVU's Institutional Review Board, the recruitment tools were amended to what is described here and the first data set was not used in the analysis.

Qualtrics, an online survey tool, was used to administer the survey and collect participant responses. Participants were not asked to give identifying information on the survey in order to keep their responses anonymous and protect their confidentiality.

Demographic criteria for participants responding to the survey was stipulated so that participants must have been at least 18 years of age, identify as Muslim, have enough fluency in English to complete the surveys, and have lived in the US for three months or more. The Qualtrics link to the survey was accompanied by a short paragraph informing potential 


\section{BARRIERS AND FACILITATORS OF SEEKING PSYCHOLOGICAL HELP}

participants about the nature of the study, researcher contact information, and compensation rate. Interested participants were directed to click the Qualtrics link to learn more about the study. Before beginning, participants read and indicated agreement to the informed consent. The informed consent included information regarding the Institutional Review Board approval for the study, risks and benefits regarding participation, anonymity of the data, notice that they may opt out of the study at any time or skip any question, and information regarding how to request the study findings.

After indicating agreement to the informed consent, participants completed the study measures including the Self-Stigma of Seeking Help Scale (SSOSH; Vogel et al., 2006), the Acculturation Rating Scale for Arabic Americans-II, Attraction to Arabic Culture Subscale (ARSAA-II; AAmC; Jadalla \& Lee, 2015), the Short Muslim Practice and Belief Scale (SMPBS; AlMarri et al., 2009), the Brief Perceived Ethnic Discrimination Questionnaire Community Version Lifetime Exposure Scale (PEDQ-CV; Brondolo et al., 2005), and the Religious Discrimination Scale (RDS; Kawika Allen et al., 2018). These measures were distributed in a random, counter-balanced order through Qualtrics to prevent order effects. Finally, participants were asked to complete the demographics questionnaire including items related to their age, gender, educational level, length of time living in the U.S., and generational status. The demographics survey was distributed at the end of the survey. After completing all measures, the participants had the option to click a link to another Qualtrics form where they could provide their email address if they wanted to be entered to win one of ten 25-dollar Amazon gift cards as compensation for completing the study. Participant email addresses were collected separately from their survey responses in order to protect anonymity. At the 


\section{BARRIERS AND FACILITATORS OF SEEKING PSYCHOLOGICAL HELP}

conclusion of data collection, ten participants who entered their emails were randomly selected and emailed a 25-dollar Amazon gift card.

The final page of the study also contained information regarding resources participants could access if the survey caused them emotional distress. Links to web-based counseling services and hotlines were included.

\section{Research Design}

The proposed study examined the relationships among acculturation, religiosity, perceived ethnic and religious discrimination, and self-stigma of seeking mental health in MUS. A quantitative, self-report survey-style, descriptive cross-sectional (correlational) research design was used to assess these questions. This research design was chosen due to the nascence of this research area and the necessity for greater research at this stage of investigation.

\section{Hypotheses and Analyses}

The following analyses were used to test each of the four hypotheses of this study:

Hypothesis 1. Higher levels of Islamic practices and beliefs will be positively associated with self-stigma of seeking psychological help in a MUS sample. Structural equation modeling, estimating a direct path between Islamic beliefs and practices, using items from the Short Muslim Practice and Belief Scale (S-MPBS; AlMarri et al., 2009), and self-stigma, using items from the Self-Stigma of Seeking Help Scale (SSSH; Vogel et al., 2006), was used to test this hypothesis.

Hypotheses 2-4. The association between Islamic practices and beliefs and self-stigma of seeking psychological help will be partially mediated by the latent constructs of acculturation to White-majority American culture, perceived ethnic discrimination, and perceived religious discrimination, controlling for demographic factors, in a sample of MUS. Structural equation 


\section{BARRIERS AND FACILITATORS OF SEEKING PSYCHOLOGICAL HELP}

modeling was used to assess this hypothesized mediation model. One exogenous variable (Islamic practices and beliefs), three mediator variables (acculturation, perceived religious discrimination, and perceived ethnic discrimination), and one endogenous variable (self-stigma of seeking psychological help) were used in this analysis. The exogenous variable, Islamic beliefs and practices, was measured using items from the Short Muslim Practice and Belief Scale (S-MPBS; AlMarri et al., 2009). The mediator variable, acculturation level toward White American culture, was measured using items from the AAmC score of the Acculturation Rating Scale for Arabic Americans-II (ARSAA-II; Jadalla \& Lee, 2015). The next mediator variable, perceived ethnic discrimination, was measured using items from the Brief Perceived Ethnic Discrimination Questionnaire - Community Version Lifetime Exposure Scale (PEDQ-CV; Brondolo et al., 2005). Perceived ethnic discrimination, the final mediator variable, was measured using items from the Religious Discrimination Scale (RDS; Kawika Allen et al., 2018). Items from the Self-Stigma of Seeking Help Scale (SSOSH; Vogel et al., 2006) were used as the endogenous variable to measure participants' experiences of self-stigma related to seeking psychological help. Control variables were used from the demographic questionnaire. The model represented in figure 2 demonstrates the model specification, or hypothesized relationships among the primary study variables. Model estimation, the calculation of fit indices and parameter estimates for a model, were also conducted.

Finally, the proposed mediation was assessed by examining the indirect effects of Islamic beliefs and practices through each of the proposed mediator variables (acculturation, perceived religious discrimination, and perceived ethnic discrimination) on self-stigma for seeking psychological help. In addition, the remaining direct effects of Islamic beliefs and practices on self-stigma of seeking psychological help were examined (see figure 2). 
BARRIERS AND FACILITATORS OF SEEKING PSYCHOLOGICAL HELP

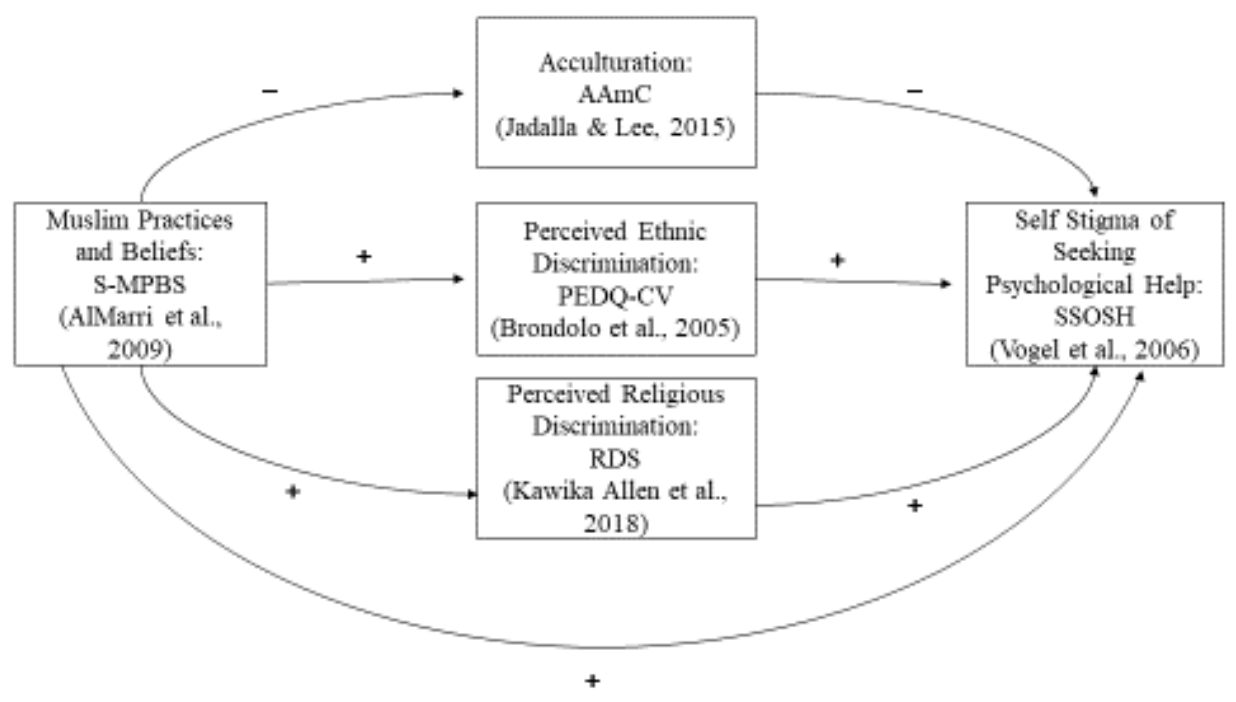

Figure 2. Model estimating the relationship between Islamic practices and beliefs and selfstigma for seeking psychological help, with acculturation, perceived racial and ethnic discrimination, and perceived religious discrimination as mediators. 
BARRIERS AND FACILITATORS OF SEEKING PSYCHOLOGICAL HELP

\section{CHAPTER 3: RESULTS}

\section{Introduction to Results}

The purpose of this study was to investigate the relationships among acculturation, religiosity, perceived ethnic and religious discrimination, and self-stigma of seeking psychological help in a sample of Muslims living in the United States (MUS). A quantitative, self-report survey-style, descriptive cross-sectional (correlational) research design was used to assess these relationships. This research design was chosen due to the nascence of research in this area and the necessity for greater research at this stage of investigation. The following hypotheses were proposed:

Hypothesis 1. Higher levels of Islamic practices and beliefs as measured by items from the Short Muslim Practice and Belief Scale (S-MPBS; AlMarri, Oei, \& Al-Adawi, 2009) will be positively related to self-stigma of seeking psychological help as measured by items from the Self-Stigma of Seeking Help Scale (SSOSH; Vogel et al., 2006) in a MUS sample.

Hypothesis 2. The association between Islamic practices and beliefs and self-stigma of seeking psychological help will be partially mediated by acculturation to American majority culture as measured by items from the Attraction to American Culture Subscale (AAmC) of the Acculturation Rating Scale for Arabic Americans-II (ARSAA-II; Jadalla \& Lee, 2015) in a MUS sample.

Hypothesis 3.The association between Islamic practices and beliefs and self-stigma of seeking psychological help will be partially mediated by perceived ethnic discrimination as measured by items from the Brief Perceived Ethnic Discrimination Questionnaire - Community Version Lifetime Exposure Scale (PEDQ-CV; Brondolo et al., 2005) in a MUS sample.

Hypothesis 4. The association between Islamic practices and beliefs and self-stigma of 


\section{BARRIERS AND FACILITATORS OF SEEKING PSYCHOLOGICAL HELP}

seeking psychological help will be partially mediated by perceived religious discrimination as measured by the items from the Religious Discrimination Scale (RDS; Kawika Allen, Wang, Richards, Ming, \& Suh, 2018) in a MUS sample.

\section{Preliminary Analyses}

Preliminary descriptive analyses were conducted to assess the data for potential univariate and multivariate outliers. Descriptive statistics, means, frequencies and standard deviations for all demographic data were calculated using the Statistical Package for Social Sciences (SPSS; IBM Corp, 2016). The final analytical sample consisted of $n=202$ participants. Initially, 308 participants opened or began to respond to the survey but 105 of these respondents did not complete the majority of the survey and their responses were deleted. Approximately 90 of the 308 respondents only opened the survey but did not complete any items. Approximately 15 of the 308 respondents completed less than 50 percent of the items. In each case, their data sets were deleted, leaving 202 participant responses in the analytical sample. One participant identified as "Christian" and their response was also deleted as they did not meet the study criteria.

Participant demographic information is reported in Table 1 below. Most of the sample consisted of individuals who identified as female (75.8\%) and Asian/Pacific Islander (46.3\%). The majority of respondents were between 20 to 29 years old (46.5\%), born in the U.S.A. (54.3\%), and Sunni (84.3\%). A little over half of respondents indicated that their primary language was English (55.8\%). Participants primarily identified as single (39.7\%) or married (38.2\%) and had a bachelor's (26.1\%), master's (28.1\%), or doctoral (25.1\%) degree. Respondents' household income was diverse, ranging from “less than $\$ 25,000$ ” (20.9\%) to " $\$ 150,000$ or more" $(19.4 \%)$ over the past 12 months. 
BARRIERS AND FACILITATORS OF SEEKING PSYCHOLOGICAL HELP

Table 1

Demographic Information

\begin{tabular}{|c|c|c|}
\hline Demographics & $N$ & Percentage \\
\hline \multicolumn{3}{|l|}{ Gender } \\
\hline Male & 48 & 24.2 \\
\hline Female & 150 & 75.8 \\
\hline \multicolumn{3}{|l|}{ Age } \\
\hline $18-19$ & 18 & 9.0 \\
\hline $20-29$ & 93 & 46.5 \\
\hline $30-39$ & 57 & 28.5 \\
\hline $40-49$ & 9 & 4.5 \\
\hline $50-59$ & 10 & 5 \\
\hline $60-69$ & 8 & 4 \\
\hline $70-79$ & 1 & 0.5 \\
\hline $80-89$ & 1 & 0.5 \\
\hline Not Provided & 4 & 1.5 \\
\hline \multicolumn{3}{|l|}{ Race/Ethnicity } \\
\hline Black/African-American & 12 & 6.1 \\
\hline Asian/Pacific Islander & 93 & 46.3 \\
\hline Caucasian/White & 7 & 3.6 \\
\hline Hispanic or Latino(a) & 0 & 0 \\
\hline Indigenous or Aboriginal & 0 & 0 \\
\hline Middle Eastern or North African & 69 & 35.2 \\
\hline Multiracial & 12 & 6.1 \\
\hline Self-Identify & 3 & 1.5 \\
\hline \multicolumn{3}{|l|}{ Born In U.S.A } \\
\hline Yes & 108 & 54.3 \\
\hline No & 91 & 45.7 \\
\hline \multicolumn{3}{|l|}{ Region of Origin } \\
\hline Asia & 87 & 44.2 \\
\hline Caribbean & 1 & 0.5 \\
\hline Central America & 0 & 0 \\
\hline Europe & 3 & 1.5 \\
\hline Middle East/North Africa & 70 & 35.5 \\
\hline Sub-Saharan Africa & 6 & 3.0 \\
\hline North America & 17 & 8.6 \\
\hline Oceania & 0 & 0 \\
\hline South America & 2 & 1.0 \\
\hline Other & 11 & 5.6 \\
\hline \multicolumn{3}{|l|}{ Length of Time in the U.S.A. } \\
\hline 3 Months to 1 Year & 3 & 1.5 \\
\hline $2-5$ Years & 17 & 8.5 \\
\hline $6-10$ Years & 11 & 5.5 \\
\hline 11-15 Years & 12 & 6.0 \\
\hline $16-20$ Years & 20 & 10.1 \\
\hline Over 21 Years & 42 & 21.1 \\
\hline
\end{tabular}




\begin{tabular}{|c|c|c|}
\hline All My Life & 94 & 47.2 \\
\hline \multicolumn{3}{|l|}{ First Language } \\
\hline English & 111 & 55.8 \\
\hline Other & 88 & 44.2 \\
\hline \multicolumn{3}{|l|}{ Relationship Status } \\
\hline Single, never married & 79 & 39.7 \\
\hline In a Relationship & 2 & 13.6 \\
\hline Engaged & 6 & 3.0 \\
\hline Married or in Domestic Partnership & 76 & 38.2 \\
\hline Widowed & 0 & 0 \\
\hline Divorced & 10 & 5.0 \\
\hline Separated & 1 & 0.5 \\
\hline \multicolumn{3}{|l|}{ Highest Completed Level of Education } \\
\hline No Schooling Completed & 0 & 0 \\
\hline Nursery School to $8^{\text {th }}$ Grade & 0 & 0 \\
\hline Some High School, No Diploma & 0 & 0 \\
\hline High School Graduate or GED & 2 & 1.0 \\
\hline Some College, No Degree & 21 & 10.6 \\
\hline Trade/Technical/Vocational Training & 0 & 0 \\
\hline Associate Degree & 6 & 3.0 \\
\hline Bachelor's Degree & 52 & 26.1 \\
\hline Master's Degree & 56 & 28.1 \\
\hline Professional Degree & 12 & 6.0 \\
\hline Doctorate Degree & 50 & 25.1 \\
\hline \multicolumn{3}{|l|}{ Household Income } \\
\hline Less than $\$ 25,000$ & 40 & 20.9 \\
\hline$\$ 25,000$ to $\$ 34,000$ & 13 & 6.8 \\
\hline$\$ 35,000$ to $\$ 49,000$ & 20 & 10.0 \\
\hline$\$ 50,000$ to 74,000 & 24 & 12.6 \\
\hline$\$ 75,000$ to $\$ 99,000$ & 21 & 11.0 \\
\hline$\$ 100,000$ to $\$ 149,000$ & 36 & 18.8 \\
\hline$\$ 150,000$ or More & 37 & 19.4 \\
\hline \multicolumn{3}{|l|}{ Religious Identity } \\
\hline Sunni & 167 & 84.3 \\
\hline Shi’a & 17 & 8.6 \\
\hline Ahmadiyya & 0 & 0 \\
\hline Ibadi & 0 & 0 \\
\hline Sufism & 2 & 1.0 \\
\hline Self-Identify Muslim & 12 & 6.1 \\
\hline
\end{tabular}

Participants were asked to rate the number of times they sought professional help for both physical concerns and mental health concerns. Participants reported higher medical professional use rates than mental health professional use rates overall (see Table 2). 
Table 2

Reported Past Use of Professional Services

\begin{tabular}{lll}
\hline Sought Professional Help for Physical Concern & & \\
Never & 40 & 20.2 \\
$1-2$ Times & 54 & 27.3 \\
$3-5$ Times & 24 & 12.1 \\
More than 5 Times & 80 & 40.4 \\
\hline Sought Professional Help for Mental Health Concern & & \\
Never & 82 & 41.4 \\
$1-2$ Times & 56 & 28.3 \\
$3-5$ Times & 22 & 11.1 \\
More than 5 Times & 38 & 19.2 \\
\hline
\end{tabular}

\section{Primary Analyses}

Measurement model. Descriptive statistics, means, frequencies and standard deviations

for all scales were calculated using the Statistical Package for Social Sciences (SPSS; IBM Corp, 2016; see tables three through seven). Five items on the SSOSH scale were reverse coded as per the scale instructions. Categorical demographic variables (relationship status, gender, birth country, primary language, ethnicity, region of origin, and religious denomination) were dummy coded for the analyses. Table 8 indicates reference groups for the dummy coded variables.

The measurement model analysis began by examining the measurement component of the SEM model to assess the performance of the observed items in measuring the latent concepts. Model fit of the latent variables (self-stigma for seeking psychological help, acculturation to U.S. culture, Islamic religious beliefs and practices, perceived ethnic discrimination, and perceived religious discrimination) was conducted using confirmatory factor analysis with Stata software (StataCorp, 2017). Factor loadings for each latent variable were evaluated and items with factor loadings of 0.60 or above were retained for use in the SEM analysis (MacCallum, Widaman, Zhang, \& Hong, 1999; MacCallum, Widaman, Preacher, \& Hong, 2001). Items that were retained are indicated in bold in Table 3 through Table 7 below. 
Table 3

Factor Loadings of Self Stigma of Seeking Psychological Help Scale

\begin{tabular}{lllc}
\hline Item & $\begin{array}{l}\text { Factor } \\
\text { Loading }\end{array}$ & Mean & $\begin{array}{c}\text { Standard } \\
\text { Deviation }\end{array}$ \\
\hline 1. I would feel inadequate if I went to a & $\mathbf{0 . 7 7 5}$ & $\mathbf{2 . 2 1}$ & $\mathbf{1 . 1 0 0}$ \\
therapist for psychological help. & & & \\
$\begin{array}{l}\text { 2. My self-confidence would NOT be } \\
\text { threatened if I sought professional help. }\end{array}$ & 0.491 & 3.134 & 1.377 \\
3. Seeking psychological help would make & $\mathbf{0 . 8 0 2}$ & $\mathbf{1 . 7 4 0}$ & $\mathbf{0 . 9 1 8}$ \\
$\begin{array}{l}\text { me feel less intelligent. } \\
\text { 4. My self-esteem would increase if I talked } \\
\text { to a therapist. }\end{array}$ & 0.353 & 2.465 & 0.934 \\
5. My view of myself would not change just \\
$\begin{array}{l}\text { because I made the choice to see a } \\
\text { therapist. }\end{array}$
\end{tabular}

Five items were retained from the Self Stigma for Seeking Help Scale (Vogel et al., 2006). In the present study, the internal consistency reliability was good (Cronbach's alpha $=$ .85) but the items retained were determined based on factor loadings only. This scale was originally developed using college students in the USA (Cronbach's alpha $=0.89$ ) and when administered to a Turkish population it did demonstrate somewhat lower, but still acceptable internal consistency reliability (Cronbach's alpha $=.82$; Vogel et al., 2013). Likewise, a sample from the United Arab Emirates also demonstrated a somewhat lower, but adequate, internal consistency reliability (Cronbach’s alpha = .77; Vogel et al., 2017). 
All but one (item seven) of the reverse scored items were not retained in the present study. This could indicate that the reverse scored items were not very clear to the participants in the present study. Upon continued analyses, many of the items that loaded less high in the present study also had lower factor loadings in validation studies across cultures. For example, item two had low factor loadings in Greece (0.47), Israel (0.47), and Turkey (0.58; Vogel et al., 2013). Item four demonstrated factor loadings between 0.31 and 0.52 in samples from England, Greece, Israel, Taiwan, Turkey, and the USA. Item five demonstrated factor loadings between 0.24 and 0.56 in samples from England, Greece, Israel, Taiwan, Turkey, and the USA (Vogel et al., 2013). Item nine demonstrated factor loadings between 0.28 and 0.50 in samples from England, Greece, Israel, Turkey, and the USA (Vogel et al., 2013). Item ten demonstrated lower factor loadings in samples from Israel (0.33), Turkey (0.30), and Taiwan (0.45) (Vogel et al., 2013). These scores indicate that cultural differences could explain the lower factor loadings found on some items of the SSOSH scale in the present study.

Table 4

Factor Loadings of Acculturation Rating Scale

\begin{tabular}{|c|c|c|c|}
\hline Item & $\begin{array}{l}\text { Factor } \\
\text { Loading }\end{array}$ & Mean & $\begin{array}{l}\text { Standard } \\
\text { Deviation }\end{array}$ \\
\hline $\begin{array}{l}\text { 1. I enjoy listening to English language } \\
\text { music }\end{array}$ & 0.443 & 4.270 & 1.211 \\
\hline 2. I speak English & 0.321 & 4.900 & 0.400 \\
\hline $\begin{array}{l}\text { 3. I enjoy English language movies } \\
\text { (American movies) }\end{array}$ & 0.467 & 4.720 & 0.618 \\
\hline 4. I associate with White Americans & 0.150 & 3.350 & 1.345 \\
\hline $\begin{array}{l}\text { 5. I enjoy English language TV (American } \\
\text { TV) }\end{array}$ & 0.606 & 4.580 & 0.8340 \\
\hline 6. My thinking is done in English & 0.640 & 4.340 & 1.047 \\
\hline 7. I write in English & 0.564 & 4.860 & 0.401 \\
\hline 8. I enjoy reading in English & 0.662 & 4.710 & 0.668 \\
\hline $\begin{array}{l}\text { 9. My contact with the USA has been (none } \\
\rightarrow \text { almost always) }\end{array}$ & 0.181 & 2.990 & 1.231 \\
\hline 10. My friends now are of White origin & 0.570 & 3.200 & 1.603 \\
\hline $\begin{array}{l}\text { 11. My friends, while I was growing up, were } \\
\text { of American origin }\end{array}$ & 0.504 & 3.670 & 1.415 \\
\hline
\end{tabular}


BARRIERS AND FACILITATORS OF SEEKING PSYCHOLOGICAL HELP

12. I like to identify myself as an American $\quad 0.015 \quad 1.470 \quad 0.985$

13. I like to identify myself as a White $\quad 0.507 \quad 4.350 \quad 0.888$ American

Only three items were retained from the Acculturation Rating Scale for Arab Americans - Attraction to American Culture Subscale (AAmC; Jadallah and Lee, 2015). The internal consistency reliability for the AAmC in the present study was adequate (Cronbach's alpha = 0.73). It is possible that the lower internal consistency reliability and fewer high factor loadings demonstrated on this scale occurred because this scale was originally developed for use with Arab Americans and the sample from the present study was much more diverse. Many of the items that had low factor loadings in the present study also displayed lower factor loadings in the scale development study. For example, in the scale development study, low factor loadings included item $13=0.148$, item $12=0.408$, item $11=0.486$, and item $10=0.500$ (Jadallah and Lee, 2015). The items that had high factor loadings in the present study also had high factor loadings in the scale development study: item five $=0.739$; item six $=0.708$; item eight $=0.686$ (Jadallah and Lee, 2015). In the scale development study, AAmC total scores were higher for English responders than Arabic responders, signifying greater acculturation for English speakers and suggesting that greater use and comfort with English can serve as an indicator of acculturation (Jadallah and Lee, 2015). 
Table 5

Factor Loadings of Short Muslim Practice and Beliefs Scale

\begin{tabular}{llll}
\hline Item & $\begin{array}{l}\text { Factor } \\
\text { Loading }\end{array}$ & Mean & $\begin{array}{l}\text { Standard } \\
\text { Deviation }\end{array}$ \\
\hline $\begin{array}{l}\text { 1. All Muslim countries should be governed } \\
\text { by absolute Shariah law. }\end{array}$ & 0.493 & 2.120 & 1.083 \\
$\begin{array}{l}\text { 2. Religion should govern all my actions. } \\
\text { 3. If someone commits a crime they should } \\
\text { be punished according to absolute Shariah }\end{array}$ & 0.644 & 3.050 & 1.363 \\
$\quad \begin{array}{l}\text { law (e.g., stoning to death for adultery). } \\
\text { 4. Men/women should not shake hands with }\end{array}$ & 0.577 & 2.260 & 1.114 \\
$\quad \begin{array}{l}\text { opposite sex in public. } \\
\text { The portrayal (picture, painting) of a }\end{array}$ & 0.397 & 2.070 & 1.172 \\
$\quad \begin{array}{l}\text { human figure (such as a face) should not } \\
\text { be allowed. }\end{array}$ & & & \\
6. I follow all the pillars of Islam. & $\mathbf{0 . 8 9 1}$ & $\mathbf{3 . 9 0 0}$ & $\mathbf{1 . 0 5 8}$ \\
$\begin{array}{l}\text { 7. I pray five times day. } \\
\text { 8. I read the Qur'an. }\end{array}$ & $\mathbf{0 . 9 1 5}$ & $\mathbf{3 . 5 5 0}$ & $\mathbf{1 . 3 9 9}$ \\
9. I fast the month of Ramadan. & $\mathbf{0 . 7 9 0}$ & $\mathbf{3 . 2 7 0}$ & $\mathbf{1 . 1 7 5}$ \\
\hline
\end{tabular}

Although item two, "Religion should govern all my actions", from the Short Muslim Practice and Beliefs Scale (S-MPBS; AlMarri et al., 2009) met criteria to be included in this analysis, it was not retained. Only the items measured by the Muslim Practices subscale (items six through nine) were used in the final analysis because they were previously demonstrated to represent the construct of Muslim practices (AlMarri, Oei, \& Al-Adawi, 2009) and it was thought better to retain only the items for that subscale for clarity and consistency. The overall internal consistency reliability for the S-MPBS in the present study was good (Cronbach's alpha = 0.89). The internal consistency reliability just for the Muslim Practices Subscale was also good $($ Cronbach's alpha $=0.89)$ in the present study 
Table 6

Factor Loadings of the Perceived Ethnic Discrimination Scale

\begin{tabular}{|c|c|c|c|}
\hline Item & $\begin{array}{l}\text { Factor } \\
\text { Loading }\end{array}$ & Mean & $\begin{array}{l}\text { Standard } \\
\text { Deviation }\end{array}$ \\
\hline $\begin{array}{l}\text { 1. Have you been treated unfairly by } \\
\text { teachers, principals, or other staff at } \\
\text { school? }\end{array}$ & 0.692 & 2.240 & 0.934 \\
\hline $\begin{array}{l}\text { 2. Have others thought you couldn't do } \\
\text { things or handle a job? }\end{array}$ & 0.643 & 2.270 & 1.131 \\
\hline $\begin{array}{l}\text { 3. Have others threatened to hurt you (ex: } \\
\text { said they would hit you)? }\end{array}$ & 0.584 & 1.490 & 0.736 \\
\hline $\begin{array}{l}\text { 4. Have others actually hurt you or tried to } \\
\text { hurt you (ex: kicked or hit you)? }\end{array}$ & 0.4307 & 1.270 & 0.600 \\
\hline $\begin{array}{l}\text { 5. Have policemen or security officers } \\
\text { been unfair to you? }\end{array}$ & 0.613 & 1.950 & 1.137 \\
\hline $\begin{array}{l}\text { 6. Have others threatened to damage your } \\
\text { property? }\end{array}$ & 0.594 & 1.380 & 0.760 \\
\hline $\begin{array}{l}\text { 7. Have others actually damaged your } \\
\text { property? }\end{array}$ & 0.525 & 1.250 & 0.546 \\
\hline $\begin{array}{l}\text { 8. Have others made you feel like an } \\
\text { outsider who doesn't fit in because of } \\
\text { your dress, speech, or other } \\
\text { characteristics related to your } \\
\text { ethnicity? }\end{array}$ & 0.704 & 2.830 & 1.241 \\
\hline $\begin{array}{l}\text { 9. Have you been treated unfairly by co- } \\
\text { workers or classmates? }\end{array}$ & 0.787 & 1.570 & 1.145 \\
\hline $\begin{array}{l}\text { 10. Have others hinted that you are } \\
\text { dishonest or can't be trusted? }\end{array}$ & 0.745 & 1.570 & 0.846 \\
\hline $\begin{array}{l}\text { 11. Have people been nice to you to your } \\
\text { face, but said bad things about you } \\
\text { behind your back? }\end{array}$ & 0.744 & 2.470 & 1.068 \\
\hline $\begin{array}{l}\text { 12. Have people who speak a different } \\
\text { language made you feel like an outsider? }\end{array}$ & 0.501 & 2.170 & 1.070 \\
\hline $\begin{array}{l}\text { 13. Have others ignored you or not paid } \\
\text { attention to you? }\end{array}$ & 0.650 & 2.450 & 1.024 \\
\hline $\begin{array}{l}\text { 14. Has your boss or supervisor been } \\
\text { unfair to you? }\end{array}$ & 0.645 & 1.840 & 0.982 \\
\hline $\begin{array}{l}\text { 15. Have others hinted that you must not } \\
\text { be clean? }\end{array}$ & 0.646 & 1.460 & 0.812 \\
\hline 16. Have people not trusted you? & 0.768 & 1.790 & 0.850 \\
\hline 17. Has it been hinted that you must be lazy? & 0.573 & 1.570 & 0.883 \\
\hline
\end{tabular}


Table 7

Factor Loadings of Religious Discrimination Scale

\begin{tabular}{|c|c|c|c|}
\hline Item & $\begin{array}{l}\text { Factor } \\
\text { Loading }\end{array}$ & Mean & $\begin{array}{l}\text { Standard } \\
\text { Deviation }\end{array}$ \\
\hline $\begin{array}{l}\text { 1. I felt disrespected because of my } \\
\text { religious views. }\end{array}$ & 0.814 & 2.750 & 0.980 \\
\hline $\begin{array}{l}\text { 2. I was ignored because I am a religious } \\
\text { person. }\end{array}$ & 0.708 & 2.340 & 0.988 \\
\hline $\begin{array}{l}\text { 3. People assumed things about me } \\
\text { because of my religion. }\end{array}$ & 0.807 & 3.600 & 1.064 \\
\hline $\begin{array}{l}\text { 4. I felt inclined to keep my religious } \\
\text { affiliation private. }\end{array}$ & 0.419 & 2.600 & 1.301 \\
\hline $\begin{array}{l}\text { 5. I was afraid of others finding out about my } \\
\text { religious beliefs. }\end{array}$ & 0.529 & 2.110 & 1.139 \\
\hline $\begin{array}{l}\text { 6. I have felt socially avoided by others due } \\
\text { to my religion. }\end{array}$ & 0.757 & 2.490 & 1.107 \\
\hline $\begin{array}{l}\text { 7. I was passed over for opportunities due } \\
\text { to my religion. }\end{array}$ & 0.609 & 2.150 & 0.991 \\
\hline $\begin{array}{l}\text { 8. I sense hostility from others because of } \\
\text { my religious affiliation. }\end{array}$ & 0.808 & 2.540 & 1.082 \\
\hline $\begin{array}{l}\text { 9. I have heard people make unfriendly } \\
\text { remarks about my religion. }\end{array}$ & 0.709 & 3.390 & 1.081 \\
\hline $\begin{array}{l}\text { 10. Others hold negative stereotypes of } \\
\text { people with my religion. }\end{array}$ & 0.717 & 3.840 & 0.982 \\
\hline $\begin{array}{l}\text { 11. I do not feel free to express who I am } \\
\text { religiously. }\end{array}$ & .663 & 2.630 & 1.206 \\
\hline
\end{tabular}

The majority of items were retained from both the Brief Perceived Ethnic Discrimination Questionnaire - Community Version Lifetime Exposure Scale (PEDQ-CV; Brondolo et al., 2005) and the Religious Discrimination Scale (RDS; Kawika Allen et al., 2018). Each of these scales behaved as expected. The internal consistency reliability for the PEDQ-CV in the present study was good (Cronbach's alpha $=0.92)$. The internal consistency reliability for the RDS for the present study was good (Cronbach's alpha $=0.905)$.

Structural model. The study hypotheses were tested using structural equation modeling with Stata software (StataCorp, 2017). Due to minimal missing data for some participants, usually only household income, 187 participants were included in this portion of the analysis so that all 


\section{BARRIERS AND FACILITATORS OF SEEKING PSYCHOLOGICAL HELP}

cases had a complete data set. Results are discussed below and are organized around each of the four hypotheses associated with this study.

Hypothesis 1. Higher levels of Islamic practices and beliefs will be positively associated with self-stigma of seeking psychological help in a MUS sample. Structural equation modeling was used to test this hypothesis. An initial model estimating a direct path between Islamic beliefs and practices using items from the Short Muslim Practice and Belief Scale (S-MPBS; AlMarri et al., 2009) and self-stigma using items from the Self-Stigma of Seeking Help Scale (SSSH; Vogel et al., 2006) was examined. The direct path between Muslim practices and self-stigma of seeking help was not significant, $\beta=-0.084, p=0.299$ (see figure 3). This hypothesis was not supported.

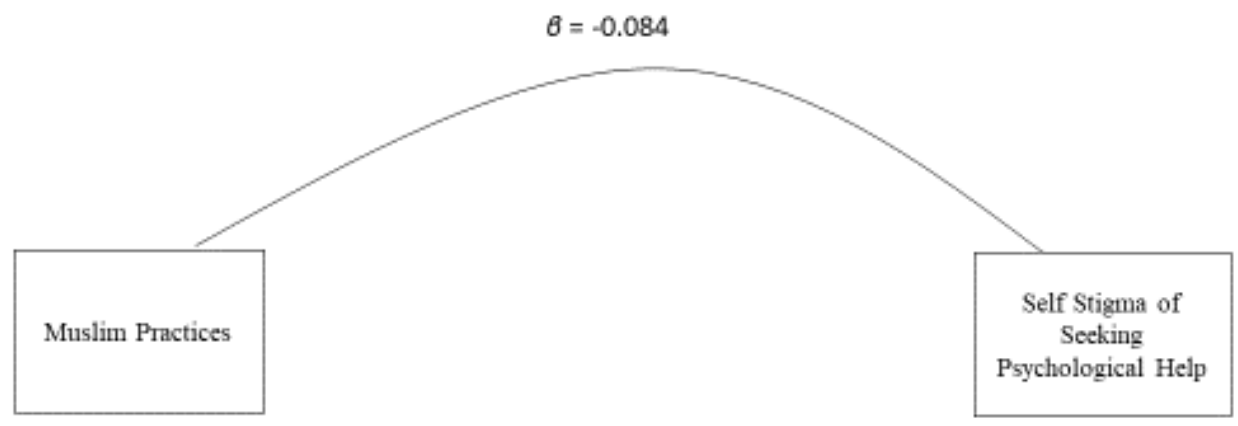

Figure 3. Standardized coefficients for direct path between Muslim practices of SSOSH.

Equation includes controls for birth country, Muslim denomination, gender, ethnicity, length of time in the U.S., relationship status, degree level, household income, past professional mental health use, and age. $N=187 ; * p<.05, * * p<.01$

Hypotheses 2-4. The association between Islamic practices and beliefs and self-stigma of seeking psychological help will be partially mediated by acculturation to American majority culture, perceived ethnic discrimination, and perceived religious discrimination, controlling for demographic factors, in a sample of Muslims living in the United States. Structural equation 


\section{BARRIERS AND FACILITATORS OF SEEKING PSYCHOLOGICAL HELP}

modeling was used to assess this hypothesized mediation model. One exogenous variable (Islamic practices and beliefs), three mediator variables (acculturation, perceived religious discrimination, and perceived ethnic discrimination), and one endogenous variable (self-stigma of seeking psychological help) were used in this analysis. The exogenous variable, Islamic beliefs and practices, was measured using items from the Short Muslim Practice and Belief Scale (S-MPBS; AlMarri et al., 2009). The mediator variable, acculturation level toward White American culture, was measured using items from the AAmC score of the Acculturation Rating Scale for Arabic Americans-II (ARSAA-II; Jadalla \& Lee, 2015). The next mediator variable, perceived ethnic discrimination, was measured using items from the Brief Perceived Ethnic Discrimination Questionnaire - Community Version Lifetime Exposure Scale (PEDQ-CV; Brondolo et al., 2005). Perceived ethnic discrimination, the final mediator variable, was measured using items from the Religious Discrimination Scale (RDS; Kawika Allen et al., 2018). Items from the Self-Stigma of Seeking Help Scale (SSOSH; Vogel et al., 2006) were used as the endogenous variable to measure participants' experiences of self-stigma related to seeking psychological help. Each variable was measured using the items indicated through factor analysis (see Tables 3 - 7 above). Demographic variables were entered in the structural model at each path in order to control for potential confounding effects. The control variables were birth country, Muslim denomination, gender, ethnicity, length of time in the U.S., relationship status, degree level, household income, and age.

Next, model estimation, the calculation of fit indices and parameter estimates for the model, were conducted. Stata software (StataCorp, 2017) was used for model estimation using a two-step process. First, fit indices, including the chi-square test of model fit, the root mean 


\section{BARRIERS AND FACILITATORS OF SEEKING PSYCHOLOGICAL HELP}

square error of approximation (RMSEA), comparative fit index (CFI), and standardized root mean square residual (SRMR) were calculated (Kenny, 2015).

The value of the chi-square test of model fit for a just-identified model is zero with no degrees of freedom, indicating perfect fit (Kline, 2005). As the model chi-square value increases it indicates a more over-identified model with poorer fit (Kline, 2005). As a "badness of fit" index, the model chi-square tests the null hypothesis that the model has perfect fit in the population. For the purposes of this analysis, a model chi-square value with an alpha level $\leq .05$ was considered significant, indicating poor model fit (Kline, 2005).

The RMSEA is a parsimony-adjusted index that favors simpler models and does not require a null hypothesis because it approximates a non-central chi-square distribution. The RMSEA is also a "badness of fit" index such that a value of zero represents the best fit with higher values indicating worse fit (Kline, 2005). In the present study, a RMSEA $\leq .05$ was taken to indicate a close approximate fit, reasonable error approximation was considered of values between .05 and .08 , and RMSEA $\geq .10$ was deemed poor fit (Kline, 2005).

The CFI assesses the relative fit of the proposed model with a baseline model, the null model, which assumes zero population variance among the observed variables (Kline, 2005). CFI values greater than .90 were taken to indicate reasonably good fit of the model (Kline, 2005). Finally, the SRMR, a measure of the mean absolute correlation residual, was computed by transforming the sample covariance matrix and the predicted covariance matrix into correlation matrices. Values of SRMR of .10 or less were considered a favorable model fit (Kline, 2005). Based on the model fit analyses, theoretically justified modifications may be made to parameter estimates to improve model fit (Kline, 2005). 


\section{BARRIERS AND FACILITATORS OF SEEKING PSYCHOLOGICAL HELP}

Overall, estimates of model fit indicated an adequate to good model fit. A chi-square test of independence indicated poor model fit $X^{2}(745, N=187)=1307.06, p=0.001$; however, this test is sensitive to larger sample sizes and other indices are better indicators of model fit with the larger sample size of the present study (Kline, 2005). The root mean square error of approximation $(\mathrm{RMSEA}=0.06)$ indicated reasonable error approximation and model fit. The standardized root mean square residual, $(\mathrm{SRMR}=0.06)$ indicated a good model fit. The comparative fit index indicated an adequate model fit $(\mathrm{CFI}=0.84)$.

Next the proposed mediation was assessed by examining the indirect effects of Islamic beliefs and practices through each of the proposed mediator variables (acculturation, perceived religious discrimination, and perceived ethnic discrimination) on self-stigma for seeking psychological help. In addition, the remaining direct effects of Islamic beliefs and practices on self-stigma of seeking psychological help were examined. Standardized coefficients for all paths estimated in the model, including for the control measures, are shown in Table 8 and are represented in Figure 3. Parameter estimates significant at the $\alpha<.05$ level were considered statistically significant.

Only the paths from Muslim practices to acculturation $(\beta=-0.164, p=.038)$ and from acculturation to self-stigma of seeking psychological help $(\beta=-0.471, p=.001)$ were statistically significant from the hypothesized model. Higher Muslim practice scores were associated with lower levels of acculturation, controlling for other variables. Lower acculturation was associated with higher levels of self-stigma for seeking psychological help, controlling for other variables.

Next, the indirect effects path was analyzed to determine whether the path between Islamic beliefs and practices and self-stigma of seeking psychological help was mediated by acculturation. No significant indirect effect was found $(\beta=0.077, p=0.095)$. Hypotheses two 


\section{BARRIERS AND FACILITATORS OF SEEKING PSYCHOLOGICAL HELP}

through four were rejected. Perceived religious discrimination and perceived ethnic discrimination were found to covary $(\beta=0.815, p=0.00)$ and this covariance is depicted in figure 8 .

Analyses of the control variables indicated that identifying as Sunni Muslim was associated with reports of higher self-stigma of seeking psychological help $(\beta=0.158, p=$ 0.031), controlling for other variables. Higher degree level $(\beta=-0.187, p=0.024)$ and higher past usage of mental health services $(\beta=-0.267, p<0.001)$ were associated with lower selfstigma of seeking psychological help, controlling for the other variables. Identifying as female was associated with greater levels of perceived religious discrimination $(\beta=-0.204, p=0.006)$, controlling for other variables. Identifying as Asian/Pacific Islander was associated with less perceived religious discrimination $(\beta=-0.190, p=0.008)$, controlling for other variables. Greater length of time living in the USA was associated with higher levels of acculturation $(\beta=$ $0.512, p<0.001)$, higher levels of perceived religious discrimination $(\beta=0.275, p=0.005)$, and higher levels of perceived ethnic discrimination $(\beta=0.275, p=0.009)$, controlling for other variables. Older individuals were associated with lower acculturation rates $(\beta=-.0224, p=$ 0.009), controlling for other variables. Relationship status, household income, whether a participant was born in the USA, and identifying as Middle Eastern/North African were not found to be associated with any of the main study variables. 
BARRIERS AND FACILITATORS OF SEEKING PSYCHOLOGICAL HELP

Table 8

Standardized Path coefficients for Structural Equation Model (N=187)

\begin{tabular}{|c|c|c|c|c|}
\hline & \multicolumn{4}{|c|}{$\begin{array}{l}\text { Structural Equation Model Outcomes } \\
\end{array}$} \\
\hline $\begin{array}{l}\text { Structural Equation Model } \\
\text { Predictors }\end{array}$ & $\begin{array}{c}\text { Self-Stigma } \\
\text { of Seeking } \\
\text { Psychological } \\
\text { Help }\end{array}$ & Acculturation & $\begin{array}{c}\text { Perceived } \\
\text { Religious } \\
\text { Discrimination }\end{array}$ & $\begin{array}{c}\text { Perceived } \\
\text { Ethnic } \\
\text { Discrimination }\end{array}$ \\
\hline Acculturation & $-0.471 * *$ & -- & -- & -- \\
\hline $\begin{array}{l}\text { Perceived Religious } \\
\text { Discrimination }\end{array}$ & -0.047 & -- & -- & -- \\
\hline $\begin{array}{l}\text { Perceived Ethnic } \\
\text { Discrimination }\end{array}$ & 0.150 & -- & -- & -- \\
\hline Muslim Practices & -0.147 & $-0.164 *$ & -0.126 & -0.117 \\
\hline Controls & & & & \\
\hline Have seen Therapist & $-0.276 * *$ & -- & -- & -- \\
\hline $\begin{array}{l}\text { Born in USA (reference: Born } \\
\text { outside USA) }\end{array}$ & 0.158 & 0.056 & -0.010 & -0.098 \\
\hline $\begin{array}{l}\text { Sunni (reference: other } \\
\text { Muslim) }\end{array}$ & $0.158 *$ & 0.094 & 0.049 & 0.018 \\
\hline Male (reference: female) & -0.018 & -0.147 & $-0.204 * *$ & -0.081 \\
\hline $\begin{array}{l}\text { Asian/Pacific Islander } \\
\text { (reference: all other ethnicities) }\end{array}$ & -0.150 & -0.056 & $-0.190 * *$ & -0.081 \\
\hline $\begin{array}{l}\text { Middle Eastern/North African } \\
\text { (reference: all other ethnicities) }\end{array}$ & 0.021 & 0.053 & 0.062 & 0.056 \\
\hline Length of Time in USA & 0.101 & $0.512 * *$ & $0.275^{* *}$ & $0.275^{* *}$ \\
\hline In a Relationship & 0.071 & -0.021 & -0.030 & 0.001 \\
\hline Degree Level & $-0.187 *$ & 0.131 & -0.023 & -0.018 \\
\hline Household Income & -0.065 & 0.110 & -0.047 & -0.073 \\
\hline Age & 0.008 & $-0.224 * *$ & -0.144 & -0.038 \\
\hline
\end{tabular}

Note: correlated error terms: Perceived Religious Discrimination - Perceived Ethnic

Discrimination $(\beta=0.815, p<.001) ;{ }^{*} p<.05, * * p<.01$ 


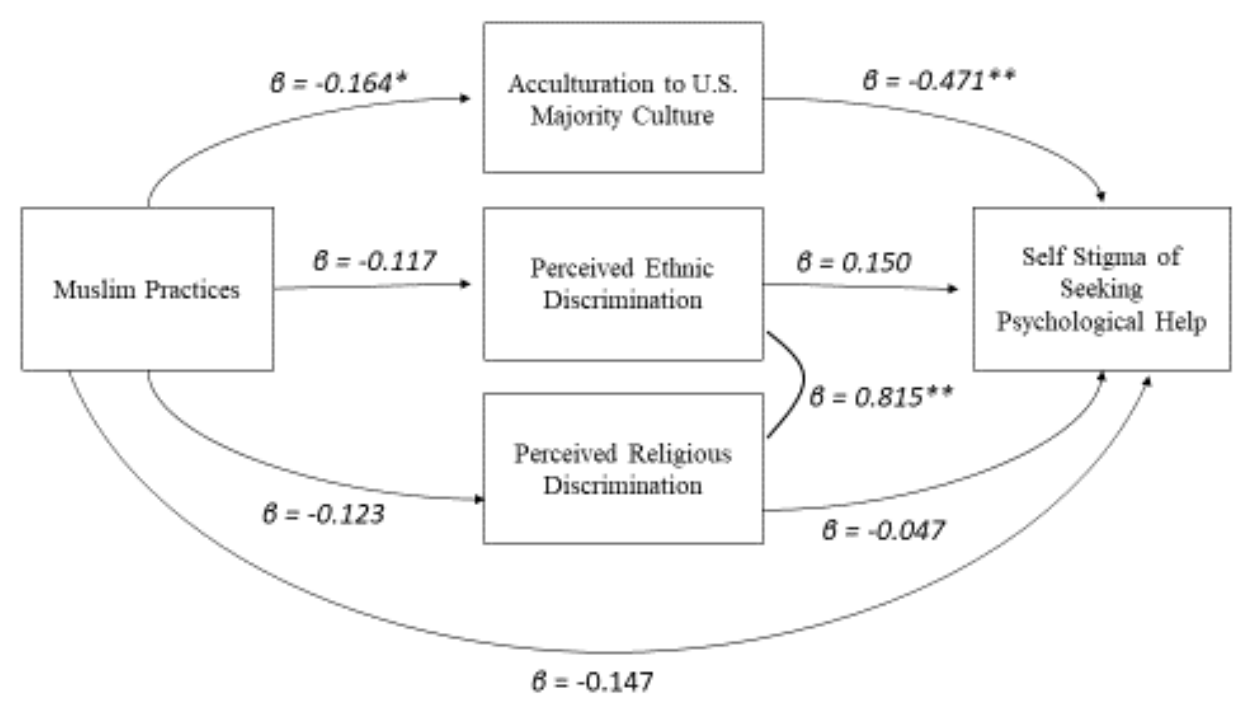

Figure 4. Standardized coefficients for key paths in structural equation model. Each equation includes controls for birth country, Muslim denomination, gender, ethnicity, length of time in the U.S., relationship status, degree level, household income, professional mental health past use, and age. See Table 8 for path estimates for these predictors. Error term for religious and ethnic discrimination allowed to covary. $n=187 ; * \mathrm{p}<.05, * * \mathrm{p}<.01$

\section{Summary}

In summary, the direct path between Muslim practices and self-stigma of seeking psychological help was not significant and that hypothesis was not supported. In addition, mediation analysis using structural equation modeling showed no significant mediated relationships among any of the hypothesized study variables, thereby rejecting hypotheses two through four. However, the paths from Muslim practices to acculturation $(\beta=-0.164, p=.038)$ and from acculturation to self-stigma of seeking psychological help $(\beta=-0.471, p=.001)$ were significant from the hypothesized model. Higher Muslim practice scores were associated with 


\section{BARRIERS AND FACILITATORS OF SEEKING PSYCHOLOGICAL HELP}

lower levels of acculturation, controlling for other variables. Lower acculturation was associated with higher levels of self-stigma for seeking psychological help, controlling for other variables. Furthermore, perceived religious discrimination and perceived ethnic discrimination were found to covary.

Analyses of the study variables indicated several significant relationships, controlling for all other study variables. Identifying as Sunni Muslim was associated with reports of higher selfstigma of seeking psychological help. Higher degree level and higher past use of mental health services were associated with lower self-stigma of seeking psychological help. Identifying as female was associated with greater levels of perceived religious discrimination. Identifying as Asian/Pacific Islander was associated with less perceived religious discrimination. Greater length of time living in the USA was associated with higher levels of acculturation, higher levels of perceived religious discrimination, and higher levels of perceived ethnic discrimination. Participant age (older individuals) was associated with lower acculturation rates. Relationship status, household income, whether a participant was born in the USA, and identifying as Middle Eastern/North African were not found to be associated with any of the main study variables. These findings and how they relate to the literature on these topics will be discussed further in the next chapter. 


\section{BARRIERS AND FACILITATORS OF SEEKING PSYCHOLOGICAL HELP}

\section{CHAPTER 4: DISCUSSION}

The current study explored barriers and facilitators of psychological help seeking for Muslims living in the United States (MUS). It was hypothesized that Muslim beliefs and practices would increase self-stigma for seeking psychological help and that this relationship would be mediated by acculturation, perceived religious discrimination, and perceived ethnic discrimination. Structural equation modeling was used to test these hypotheses. None of the relationships behaved as expected. The direct path between Muslim practices and self-stigma of seeking psychological help was not statistically significant. None of the hypothesized mediated paths were found to be statistically significant. However, the path from Muslim practices to acculturation was statistically significant and indicated that greater levels of Muslim practices were associated with lower levels of acculturation, controlling for all other study variables. The path from acculturation to self-stigma of seeking help was also statistically significant and indicated that lower levels of acculturation were associated with higher levels of self-stigma for seeking psychological help in a sample of MUS. A number of demographic variables were found to be associated with the main study variables. This chapter will discuss these findings and provide possible explanations for the results, limitations of the current study, and conclude with recommended future directions in this area of study.

\section{Discussion of the Direct Path between Muslim Practices and Self-Stigma (Hypothesis 1)}

It was hypothesized that Muslim practices and beliefs would have a direct negative effect on self-stigma of seeking psychological help. While the relationship was found to have a weak negative association, it was not statistically significant. This finding indicates that, in this sample, MUS with higher levels of religious beliefs and practices did not have statistically significant associations with self-stigma for seeking psychological help. This finding could 


\section{BARRIERS AND FACILITATORS OF SEEKING PSYCHOLOGICAL HELP}

mean that there is no relationship between these variables; however, it also could have emerged because Muslim practices and beliefs are affected by other variables in the model like whether someone was born in the U.S. or length of time spent in the U.S. Structural equation modeling does not test the effect of the study variables on the exogenous variable (Muslim practices and beliefs) due to the way hypothesized paths are examined in the analyses and this leaves some potential relationship paths unexamined. For example, Muslims who engage in greater levels of religious practice could tend to be born outside of the USA and live in the USA for a shorter period of time so once those variables are controlled for the effect of Muslim practices on selfstigma of seeking help could have been lost.

Similarly, with more participants, the relationship between Muslim practices and selfstigma of seeking psychological help may have been found significant due to increased power and a more diverse sample. The majority of the present sample consisted of individuals who identified as female (75.8\%) and Asian/Pacific Islander (46.3\%), were between 20 to 29 years old (46.5\%), born in the U.S.A. (54.3\%), and identified as Sunni (84.3\%). A little over half of respondents indicated that their primary language was English (55.8\%). Participants primarily identified as single $(39.7 \%)$ or married $(38.2 \%)$ and had a bachelor's $(26.1 \%)$, master's $(28.1 \%)$, or doctoral (25.1\%) degree. Respondents' household income was diverse, ranging from "less than $\$ 25,000 "(20.9 \%)$ to " $\$ 150,000$ or more" (19.4\%) over the past 12 months. Many of the participants were recruited from American Psychological Association list-serves and almost 60\% reported that they had received mental health treatment in the past. It is possible that the findings could have been impacted by these characteristics. For example, due to the high number of participants in this sample who received past mental health services, it is likely that self-stigma of seeking psychological help may have been neutralized. 


\section{BARRIERS AND FACILITATORS OF SEEKING PSYCHOLOGICAL HELP}

Aloud and Rathur's (2009) model of mental health help-seeking pathways and modifying factors among Arab Muslim populations (Help-Seeking Pathways of Arab Muslims, HSPAM) asserted that cultural and religious dimensions play a key role in seeking and accessing formal mental health services. They noted that help-seeking behaviors for Arab Muslim individuals can be assessed in three stages: 1) problem awareness and recognition, 2) decision to seek help, and 3) service selection. In the HSPAM model, Arab and Islamic culture and demographic factors (i.e., religion, gender, education, age, and income) operate on each of these stages. Cultural and traditional beliefs and definitions of mental illness, knowledge of mental health problems and treatment, and Arab health styles influence stage one (problem recognition). Perceived societal stigma towards mental illness, negative attitudes towards provider influence, and availability of alternative resources such as family and friends are thought to influence stage two (decision to seek help). Stage three (service selection) is thought to be influenced by acculturation level, awareness of formal services, use of informal resources, and economic and institutional barriers (Aloud \& Rather, 2009). Cultural beliefs about mental health, familiarity with formal mental health services, perceived societal stigma, and help-seeking preferences predicted Arab Muslim attitudes towards formal mental health services (Aloud \& Rather, 2009).

The culture of the present sample may have been more favorable towards mental health services due to recruitment procedures, higher level of education, and high reported past mental health service usage. The measurement component could have also impacted the findings regarding this relationship because the Muslim beliefs subscale was not included in the analyses due to lower factor loadings of most items.

However, it is also possible that Muslim beliefs and practices do not effect self-stigma of seeking psychological help for other reasons. Public and societal stigma regarding mental health 


\section{BARRIERS AND FACILITATORS OF SEEKING PSYCHOLOGICAL HELP}

concerns may not be internalized by this population as self-stigma. Lower usage rates of mental health services by MUS could instead be related to cultural mistrust of typically Western counselors. Previous literature has noted that many American Muslims are reluctant to go to Western counselors due to concerns that the counselor will not understand their values and will instead try to impart Western values (Kelly et al., 1996). In one study, Muslims were found to be generally more conservative, conventional, and traditional in their approach to life than the professional counselors surveyed (Kelly et al., 1996). In Aloud and Rather's (2009) study, cultural and traditional beliefs regarding mental health were related to increased shame and decreased knowledge regarding seeking formal mental health services. Participants reported that

they were more likely to seek mental health help from doctors, family, and religious leaders over mental health professionals. It is possible that the decision whether to seek professional mental health services is made based on what resources an individual believes will be helpful, rather than self-stigma.

\section{Discussion of the Hypothesized Mediated Paths (Hypotheses 2-4)}

It was also hypothesized that the relationship between Muslim beliefs and practices and self-stigma of seeking psychological help would be mediated by acculturation. While this path was not found to be statistically significant, both the direct relationships between Muslim practices and acculturation, and between acculturation and self-stigma of seeking psychological help were found to be statistically significant. Higher Muslim practice scores were associated with lower levels of acculturation and lower acculturation scores were associated with higher levels of self-stigma for seeking psychological help. Acculturation, the process of adoption and immersion of the adoptive society or retention of the ethnic society of origin, seems to play a role in both Muslim practices and self-stigma of seeking psychological help. 


\section{BARRIERS AND FACILITATORS OF SEEKING PSYCHOLOGICAL HELP}

The items retained to measure acculturation in this study, following the factor analysis, primarily focused on thinking, reading, and watching television in English. English proficiency is often used as a proxy to measure acculturation and has been shown to be a reliable measure of acculturation, especially among foreign-born populations (Amri \& Bemak, 2013; Jadalla \& Lee, 2012; Lee, Nguyen, \& Tsui, 2011; Thomson \& Hoffman-Goetz, 2009). Proxy measures of acculturation such as language of interview are often used in research because of their convenience and reliable correlation with acculturation measurement scales, although the data they provide is often less nuanced, focusing only on a behavioral observation but not attitudes or beliefs (Thomson \& Hoffman-Goetz, 2009).

In the present study, an acculturation scale was used but the only items that displayed adequate factor loadings were related to use of English language. Results indicated that those who were less likely to think in, read, and consume English media demonstrated higher rates of Muslim practices, controlling for other variables. Often in samples less acculturated to Western culture, Muslim individuals attribute mental illness to religious or spiritual forces rather than biomedical or intrapsychic sources (Al-Adawi et al., 2002; Aloud \& Rathur, 2009). Less acculturated Muslim individuals often seek out informal or traditional help, such as family, religious leaders, friends, or physicians for mental health problems rather than formal psychological services (Aloud \& Rather, 2009). Scholars have proposed that the hesitancy to seek formal mental health services by Muslim individuals may be due largely to stigma of mental health services and seekers as well as cultural mistrust of mental health counselors and the mental health system (Ali \& Milstein, 2012; Amri \& Bemak, 2013; Kelly et al., 1996; Ciftci et al., 2013). Although the present study did not demonstrate a pathway between Muslim 


\section{BARRIERS AND FACILITATORS OF SEEKING PSYCHOLOGICAL HELP}

practices and self-stigma of seeking help, acculturation did play a role in each of these variables, lending some support to these proposals.

Higher levels of thinking in, reading, and consuming English media (acculturation) were associated with lower levels of self-stigma of seeking psychological help in the present study. Previous research has demonstrated that Arabic-speaking individuals seek psychiatric help at lower rates than English-speaking people (Youssef \& Deane, 2006). Traditionally, other sources of help, such as family, religious leaders, traditional healers, and doctors, are more frequently sought out by this population. Help-seeking by MUS who are less acculturated is likely related to a variety of factors including low awareness of services, different cultural conceptions of mental illness and treatment options, and lack of access to culturally competent clinicians (Youssef \& Deane, 2006). Based on the present study, it also appears that acculturation level, or level of comfort with English, contributes to self-stigma of seeking psychological help and may impact treatment usage and selection. While acculturation was not found to mediate the relationship between Muslim practices and self-stigma of seeking psychological help, it is possible that with a larger and more diverse sample, this pathway would have been statistically significant, and this path could merit further investigation.

No support was found for hypotheses three or four which proposed that the relationship between Muslim practices and beliefs and self-stigma of seeking psychological help would be mediated by perceived ethnic discrimination and perceived religious discrimination. None of the relationships in those paths were statistically significant. Of note, the relationships between Muslim practices and perceived ethnic and religious discrimination both demonstrated weak negative associations, had they been statistically significant, which is opposite the positive relationship that was hypothesized. Additionally, longer time spent in the U.S. was associated 


\section{BARRIERS AND FACILITATORS OF SEEKING PSYCHOLOGICAL HELP}

with higher levels of perceived discrimination. These results could be understood in light of a study conducted by Ghaffari and Citfci (2010) which examined the relationship between religiosity and self-esteem in Muslims immigrating to the United States. When faced with perceived discrimination, Muslim immigrants were more likely to turn to and identify more strongly with their religion. They noted that although positive ingroup membership is often related to higher self-esteem and reduced negative outcomes in the face of discrimination, this is not always the case. They found that perceived discrimination moderates the relationship between religiosity and self-esteem such that greater levels of perceived discrimination, regardless of high religious identity and ingroup membership, are related to decreases in selfesteem. Thus, perceived discrimination affects the direction and strength of the relationship between religious attitudes and behaviors with self-esteem (Ghaffari \& Citfci, 2010). It is possible that higher perceived religious and ethnic discrimination in the present study was also associated with stronger identification with individuals' religion.

A strong, statistically significant covariance was found between perceived ethnic and religious discrimination indicating that these variables are closely related. This finding supports the proposal that discrimination operates as an intersecting system in which marginalized identities play a simultaneous and often compounding role (Ciftci et al., 2013; Fiske, 2014). For individuals with multiple marginalized identities, the experience of discrimination may be magnified or more complex (Ciftci et. al., 2013). An intersectional lens should be used to conceptualize attitudes and behaviors related to seeking mental health treatment given the complex relationships at play between different marginalized identities such as race, gender, ethnicity, and religion (Ciftci et al., 2013). In the case of the present study, individuals reported double discrimination, experiencing discrimination related to both their ethnic identity and 


\section{BARRIERS AND FACILITATORS OF SEEKING PSYCHOLOGICAL HELP}

religious identity (Gary, 2005). When conceptualizing discrimination from an intersectional framework, it is understood that the effects differ by both degree (amount) and kind (type and effect) based on one's identities, such that the amount and negative effects of discrimination are often greater for those with higher numbers of marginalized identities (Ciftci et al., 2013). Demographic differences such as class, educational attainment, religious beliefs, and gender have all been found to impact the way members of ethnic and cultural minority groups experience discrimination and stigma in the U.S. (Ciftci et al., 2013).

Muslim women in particular have been noted to experience greater collective discrimination than their male counterparts because their clothing often communicates their religion outwardly (Ryan, 2011). Discourses in the political arena and in the media are often heavily gendered and focus on outward signs of Islam, such as the hijab worn only by women. This often results in increased prejudice and discrimination directed at Muslim women compared to their male counterparts (Ryan, 2011). These findings bore out in the present study as identifying as female was associated with higher incidence of religious discrimination, controlling for other variables.

\section{Strengths and Limitations}

This research study extended the knowledge regarding barriers and facilitators of seeking psychological help for Muslims living in the United States. The study empirically investigated elements of the HSPAM Model and provided support for the impact of culture on help seeking stigma. The use of the Religious Discrimination Scale for the first time with this population was a strength of the study and illuminated the relationship between religious discrimination and ethnic discrimination for this population. The findings from this study also reiterated the importance of the availability of culturally appropriate mental health services. 


\section{BARRIERS AND FACILITATORS OF SEEKING PSYCHOLOGICAL HELP}

While the current findings add to the scholarship on barriers and facilitators of MUS psychological help-seeking and self-stigma, there are some limitations that should be noted.

First, convenience sampling was used to recruit participants which could limit external validity to the MUS population at large. Some of the major advertising avenues for the present study were through the American Psychological Association list-serves, U.S. psychology graduate programs, Muslim Student Associations at all U.S. flagship universities, and social media sampling. These sampling procedures skewed towards college students and those in the psychological field. In addition, the sample was majority female, and all had to read English to complete the survey. As noted, MUS are a heterogeneous group and demonstrate considerable diversity on a number of dimensions such as Islamic tradition (e.g., Sunni, Shi'ite, Sufi), country of origin (e.g., native born or immigrant), pathway to Islam (e.g., convert to Islam or born into the faith), race (e.g., African American, Arab, South Asian, White, etc.), ethnicity (representing over 80 countries) socioeconomic status, and language (Amer \& Bagasra, 2013; Read, 2008; United States Department of State, 2009). The results from the present study should not be taken to fully generalize to all MUS but instead illuminate a portion of the MUS experience.

The measures used in the present study may have also impacted the results. In particular, the Attraction to American Culture (AAmC) of the Acculturation Rating Scale for Arabic Americans-II (ARSAA-II; Jadalla \& Lee, 2015) was created and normed with Arab American participants who were predominately, but not all, Muslim. This scale was chosen because it had the most cultural validity of the measures available at the time for MUS; however, the present sample was not limited to those of Arab descent and was limited only to Muslims, two differences from the validation sample, which could have contributed to some measurement error. The internal consistency reliability of the AAmC in the validation study was good 


\section{BARRIERS AND FACILITATORS OF SEEKING PSYCHOLOGICAL HELP}

$($ Cronbach's alpha $=.89)$, but in the present study it did not perform as well (Cronbach's alpha $=$ 0.73). Additionally, only three items were retained from the AAmC in the present study and each of these were related to English usage: thinking and reading in English and consuming English media. It is likely that the AAmC items retained in the present study did not fully capture the construct of acculturation for this group. Instead since the retained items pertained to English usage and comfort, that served as a proxy for acculturation. Although English proficiency is often used as a proxy to measure acculturation and has been shown to be a reliable measure of acculturation, especially among foreign-born populations (Amri \& Bemak, 2013; Jadalla \& Lee, 2012; Lee, Nguyen, \& Tsui, 2011; Thomson \& Hoffman-Goetz, 2009) the data provided is often less nuanced, focusing only on a behavioral observation but not attitudes or beliefs (Thomson \& Hoffman-Goetz, 2009).

The Short Muslim Practice and Beliefs Scale (S-MPBS; AlMarri et al., 2009) may have also presented a measurement error limitation for the present study. This 9-item Likert scale measure was designed to differentiate between participants with more relaxed versus dogmatic Islamic beliefs. Four items were related to principles of religious practice based on the pillars of Islam and five items related to general religious belief and flexibility. Only the items related to principles of religious practice were retained in the present study due to low factor loadings for most of the Islamic beliefs items. In addition, approximately five participants emailed the author to express discomfort with items one and three of the S-MPBS that related to Sharia Law, noting that these questions demonstrated poor understanding of Islam and engendered mistrust of the research study. These questions may have influenced the perceptions of the survey respondents and may have also contributed to lower response rate. 


\section{BARRIERS AND FACILITATORS OF SEEKING PSYCHOLOGICAL HELP}

While the use of structural equation modeling (SEM) is robust and helpful to analyze the structural relationship between measured variables and latent constructs and is a form of causal modeling, it is ultimately a correlational, not causational analysis (Kline, 2005). Therefore, a limitation of the present study is that no causal statements can be made from these results. Additionally, the minimum sample size of 200 observations recommended for SEM was obtained for the present study, but a larger sample size may have contributed to greater statistical power and could have increased the likelihood that statistically significant results were found. Finally, SEM is a confirmatory technique meaning that the hypothesized model must be specified a priori and then tested based on the variables measured and the sample collected. SEM does not test the effect of the study variables on the exogenous variable (Muslim practices and beliefs) so it is possible that other specified paths could have been a better fit for the data (Kline, 2005).

\section{Implications}

The findings from the present study provide important implications for counseling psychologists. Muslim practices were associated with reduced acculturation and lower acculturation was related to greater self-stigma for seeking psychological help, controlling for other variables. With this in mind, counseling psychologists should offer services in culturally appropriate ways. Clement et al. (2015) recommend clinicians provide care in community centers and general medical settings to normalize psychological services, make them more accessible, and situate them in culturally relevant settings. In addition, counseling psychologists should use terms and labels that reflect clients' culture and conceptualization of their problems. For example, in the present study, many participants were concerned with the terminology of "Sharia Law" used in the S-MPBS and wrote to the author to express distrust of the study due to 


\section{BARRIERS AND FACILITATORS OF SEEKING PSYCHOLOGICAL HELP}

the use of that term which conveyed a flat few of Islam. This demonstrates the nuance and impact that culturally appropriate or inappropriate terminology and language can carry.

Counseling psychologists should strive to use terms familiar and comfortable to MUS, and when less sensitive words are used, psychologists should apologize and strive to adjust based on client feedback.

Based on the finding that acculturation (familiarity with English) was related to less selfstigma of seeking help, counseling psychologists should strive to familiarize themselves with culturally appropriate language for MUS. This will serve to engender greater trust with Muslim clients and to avoid microaggressions both in clinical, consulting, and research activities. When possible, therapeutic services and research should be conducted in the native language of the target population in order to address discomfort, reduce self-stigma of seeking help, and make services more accessible.

When held by the client, counseling psychologists would benefit from striving to affirm a God-centric view of healing in which healing is accessed directly through prayer or reading the Qur'an or indirectly through people such as imams, doctors, family, or the mental health provider. Religious and spiritual coping can be a powerful way to cope with and give life meaning and constitutes an important cultural practice for many (Bhui et al., 2008). Maintaining this perspective and integrating it with evidence-based practice could facilitate greater trust in the therapeutic relationship and be more conducive to therapeutic change and reduced stigma.

In Muslim communities, the imam is frequently used to fulfill psychological counseling roles in place of trained mental health providers even though many imams lack formal education or training in counseling or therapy (Padela et al., 2012). Likewise, mental health providers may lack appropriate cultural understanding and skills regarding the important role religion and 


\section{BARRIERS AND FACILITATORS OF SEEKING PSYCHOLOGICAL HELP}

spirituality can play for Muslims in coping and healing from mental and physical health concerns (Bhui et al., 2008). It is recommended that counseling psychologists engage in collaborations with imams and other religious leaders to help foster greater cultural understanding for mental health care providers and instill increased counseling skills and referral networks for imams and other religious leaders (Bhui et al., 2008).

Implications for advocacy can also be taken from the present study. The findings from this study indicate that religious and ethnic discrimination are closely related. This supports Gary's (2005) assertion that individuals from under-represented and minority populations often face "double stigma", experiencing prejudice and discrimination related to multiple aspects of their identities in a compounding force. The results from the present study lend further support to the idea that research and clinical work must consider individuals from an intersectional framework. The effect of stigma differs by both degree (amount) and kind (type and effect) based on one's identities, such that the amount and negative effects of stigma are often greater for those with higher numbers of marginalized identities (Ciftci et al., 2013). Female Muslims are a good example of the double stigma principle as they may experience discrimination both for their gender and for their religion (which may be more apparent to outsiders based on whether they wear a head covering). In the present study, women did report greater levels of religious discrimination than their male counterparts, controlling for other variables, which lends some support for this idea. Advocacy efforts should continue to address negative stereotypes and discrimination based on individuals' identities.

Demographic differences such as class, educational attainment, religious beliefs, and gender have all been found to impact the way members of ethnic and cultural minority groups experience and perpetrate mental illness stigma in the U.S. (Ciftci et al., 2013). In the present 


\section{BARRIERS AND FACILITATORS OF SEEKING PSYCHOLOGICAL HELP}

study this bore out as greater acculturation, higher degree level, and having seen a therapist in the past were all related to lower levels of self-stigma of seeking psychological help. In contrast, identifying as Sunni Muslim was associated with greater perceptions of self-stigma for seeking psychological help. Similarly, participants who identified Asian Pacific Islander were associated with less religious discrimination. Participants with greater length of time living in the USA reported higher levels of religious and ethnic discrimination, controlling for other variables. This could indicate that with more time in the USA, MUS become more aware of negative cultural stereotypes and experience more discrimination and stigma based on their race and religion leading to higher levels of this discrimination reported. Counseling psychologists must continue to attend to factors such as these in clinical and academic work in order to understand and address the nuanced ways individuals may be impacted in relation to their demographics.

\section{Future Directions}

The present study illuminated avenues for further exploration in future research. First, future research related to this population should attempt to gather a larger and more diverse sample of MUS than was possible in the present study. Greater collaborations with national Islamic networks and religious groups could yield a more diverse and representative sample. In particular, this sample skewed towards college educated and psychologically minded MUS and future samples should be more diverse to have greater generalizability to the population at large. Additionally, the survey for the present study was only offered in English. Whenever possible, future research should be conducted in additional languages, such as Bahasa Indonesia or Arabic, to better understand the important role language may play in choosing and accessing mental health services. 


\section{BARRIERS AND FACILITATORS OF SEEKING PSYCHOLOGICAL HELP}

In the future, researchers should consider using alternate measures than those used in the present study to assess Muslim practices and beliefs. The S-MPBS (AlMarri, Oei, \& Al-Adawi, 2009) lacked cultural validity for some participants in the belief portion of the questions, especially those questions related to Sharia law. Future research could use only the practices subscale, as was ultimately done in the present study, or find a more culturally sensitive measure. Similarly, the AAmC of the Acculturation Rating Scale for Arabic Americans-II (ARSAA-II; Jadalla \& Lee, 2015) did not perform well with this sample. Only the items related to Englishuse displayed factor loadings large enough to retain in the present study. It is recommended that a different acculturation rating scale be used or created for future research.

In contrast, the Religious Discrimination Scale (RDS; Kawika Allen et al., 2018) did perform well and this was the first time it was used with a Muslim population. It was originally validated and used with members of the Church of Jesus Christ of Latter-day Saints in the U.S. and was the only religious discrimination scale available at the time so it was chosen for this study. The RDS's good performance in the present study provides initial support for its use in additional research with MUS.

Due to the covariance between perceived religious and ethnic discrimination illuminated in the present study, it is recommended that future research continue to take a culturally informed and intersectional approach to understanding barriers and facilitators to use of formal mental health services for MUS. Future research should continue to investigate double stigma faced by individuals with multiple marginalized identities to understand the compounding role of discrimination for this population. One related variable not overtly measured in the present study, cultural mistrust, could play a role in mental health service selection for MUS and should also be further investigated. Previous literature has noted that many American Muslims are 


\section{BARRIERS AND FACILITATORS OF SEEKING PSYCHOLOGICAL HELP}

reluctant to go to Western counselors due to concerns that the counselor will not be culturally sensitive or will try to impart Western values (Kelly et al., 1996). In the present study, Muslim practices were related to lower acculturation and lower acculturation was associated with higher self-stigma of seeking psychological help. It is possible that cultural mistrust could also play a role in these relationships. Future research should explore the impact of cultural mistrust on service selection and stigma.

Finally, as noted, some of the hypothesized relationships did not behave as expected in the present study. Qualitative methodology could be an excellent starting point to further investigate the relationships proposed in the present study before conducting future path analyses. Qualitative research would allow investigators to explore research questions with more depth and nuance without restricting participants to the limited response options of quantitative methodology. Based on the responses and feedback emailed to the author of the present study, many MUS are eager to help improve access to culturally competent and sensitive mental health services and qualitative research could assist scholars in understanding this important issue with greater nuance and depth.

\section{REFERENCES}

Abuelezam, N. N., El-Sayed, A. M., \& Galea, S. (2017). Arab American health in a racially charged US. American Journal of Preventive Medicine, 52(6), 810-812. doi: 10.1016/j.amepre.2017.02.021

Abu Raiya, H., Pargament, K. I., Stein, C., \& Mahoney, A. (2007). Lessons learned and challenges faced in developing the psychological measure of Islamic religiousness. Journal of Muslim Mental Health, 2(2), 133-154. doi: 10.1080/15564900701613058 
BARRIERS AND FACILITATORS OF SEEKING PSYCHOLOGICAL HELP

Al-Adawi, S., Dorvlo, A. S., Al-Ismaily, S. S., Al-Ghafry, D. A., Al-Noobi, B. Z., Al-Salmi, A., ... \& Chand, S. P. (2002). Perception of and attitude towards mental illness in Oman. International Journal of Social Psychiatry, 48(4), 305-317. doi:

$10.1177 / 002076402128783334$

Ali, S. R., Liu, W. M., Humedian, M. (2004). Islam 101: Understanding the religion and therapy implications. Professional Psychology: Research and Practice, 35(6), 635-642. doi: $10.1037 / 0735-7028.35 .6 .635$

Ali, M. O., \& Milstein, G., (2012). Mental illness recognition and referral practices among imams in the United States. Journal of Muslim Mental Health, 1(2), 3-13. doi: 10.3998/jmmh.10381607.0006.202

AlMarri, T. S., Oei, T. P., \& Al-Adawi, S. (2009). The development of the short Muslim practiceand belief scale. Mental Health, Religion and Culture, 12(5), 415-426. doi:10.1080/13674670802637643

Aloud, N., \& Rathur, A. (2009). Factors affecting attitudes towards seeking and using formal mental health and psychological services among Arab Muslim populations. Journal of Muslim Mental Health, 4, 79-103. http://dx.doi. org/10.1080/15564900802487675

Amer, M. M., \& Bagasra, A. (2013). Psychological research with Muslim Americans in the age of Islamophobia: Trends, challenges, and recommendations. American Psychologist, 68(3), 134-144. doi: 10.1037/a0032167

Amri, S., \& Bemak, F. (2013). Mental health help-seeking behaviors of Muslim immigrants in the United States: Overcoming social stigma and cultural mistrust. Journal of Muslim Mental Health, 7(1), 43-63. doi: 10.3998/jmmh.10381607.0007.104

Barry, D. T. (2005). Measuring acculturation among male Arab immigrants in the United 
BARRIERS AND FACILITATORS OF SEEKING PSYCHOLOGICAL HELP

States: An exploratory study. Journal of Immigrant Health, 7(3), 179-184.

doi:10.1007/s10903-005-3674-9

Bhui, K., King, M., Dein, S., \& O'Connor, W. (2008). Ethnicity and religious coping with mental distress. Journal of Mental Health, 17(2), 141-151. https://doi.org/10.1080/09638230701498408

Bos, A. E., Pryor, J. B., Reeder, G. D., \& Stutterheim, S. E. (2013). Stigma: Advances in theory and research. Basic and applied social psychology, 35(1), 1-9. doi: 10.1080/01973533.2012.746147.

Brondolo, E., Kelly, K. P., Coakley, V., Gordon, T., Thompson, S., Levy, E., ... \& Contrada, R. J. (2005). The Perceived Ethnic Discrimination Questionnaire: Development and preliminary validation of a community version. Journal of Applied Social Psychology, 35(2), 335-365. doi: 10.1111/j.1559-1816.2005.tb02124.x

Brislin, R. W. (1986). The wording and translation of research instruments. In Lonner, W. J., \& Berry, J. W. (Eds.), Field Methods in Cross-Cultural Research (137-164). Thousand Oaks, CA, US: Sage Publications, Inc.

Cash, T. F., Begley, P. J., McCown, D. A., \& Weise, B. C. (1975). When counselors are heard but not seen: Initial impact of physical attractiveness. Journal of Counseling Psychology, 22(4), 273-279. doi: 10.1037/h0076730

Ciftci, A., Jones, N., \& Corrigan, P. W. (2013). Mental health stigma in the Muslim community. Journal of Muslim Mental Health, 7(1), 17-32. doi: 10.3998/jmmh.10381607.0007.102

Clement, S., Schauman, O., Graham, T., Maggioni, F., Evans-Lacko, S., Bezborodovs, N., ... \& Thornicroft, G. (2015). What is the impact of mental health-related stigma on help- 


\section{BARRIERS AND FACILITATORS OF SEEKING PSYCHOLOGICAL HELP}

seeking? A systematic review of quantitative and qualitative studies. Psychological medicine, 45(1), 11-27. doi: 10.1017/S0033291714000129

Contrada, R. J., Ashmore, R. D., Gary, M. L., Coups, E., Egeth, J. D., Sewell, A., ...Chasse, V. (2001). Measures of ethnicity-related stress: Psychometric properties, ethnic group differences, and associations with well-being. Journal of Applied Social Psychology, 31(9) 1775-1820. doi: 10.1111/j.1559-1816.2001.tb00205.x

Corrigan, P. W., Larson, J. E., \& Rusch, N. (2009). Self-stigma and the "why try" effect: Impact on life goals and evidence-based practices. World Psychiatry, 8(2), 75-81. doi: 10.1002/j.2051-5545.2009.tb00218.x

Corrigan, P. W., \& Watson, A. C. (2002). The paradox of self-stigma and mental illness. Clinical Psychology: Science and Practice, 9(1), 35-53. doi: 10.1093/clipsy.9.1.35

Corrigan, P. W., Watson, A. C., Gracia, G., Slopen, N., Rasinski, K., \& Hall, L. L. (2005). Newspaper stories as measures of structural stigma. Psychiatric services, 56(5), 551-556. doi: 10.1176/appi.ps.56.5.551

Cuellar, I., Arnold, B., \& Maldonado, R. (1995). Acculturation rating scale for Mexican Americans-II: A revision of the original ARSMA scale. Hispanic Journal of Behavioral Sciences, 17(3), 275-304. doi: 10.1177/07399863950173001

Droogsma, R. A. (2007). Redefining Hijab: American Muslim women's standpoints on veiling. Journal of Applied Communication Research, 35(3), 294-319. doi:

$10.1080 / 00909880701434299$

Fischer, E. H., \& Farina, A. (1995). Attitudes toward seeking professional psychological help: A shortened form and considerations for research. Journal of College Student Development, 36(4), 368-373. doi: 10.1037/t05375-000 


\section{BARRIERS AND FACILITATORS OF SEEKING PSYCHOLOGICAL HELP}

Fiske, S. T. (2014). Social beings: Core motives in social psychology (3rd ed.). Hoboken, NJ: Wiley.

Gary, F. A. (2005). Stigma: Barrier to mental health care among ethnic minorities. Issues in Mental Health Nursing, 26(10), 979-999. doi: 10.1080/01612840500280638

Ghaffari, A., \& Çiftçi, A. (2010). Religiosity and self-esteem of Muslim immigrants to the United States: The moderating role of perceived discrimination. International Journal for the Psychology of Religion, 20(1), 14-25. doi: 10.1080/10508610903418038

Heppner, P. P., Wampold, B. E., \& Kivlighan, D. M. (2008). Research design in counseling: Research, statistics, \& program evaluation. Belmont, CA: Cengage Learning.

IBM Corp. Released 2016. IBM SPSS Statistics for Macintosh, Version 24.0 [Computer Software]. Armonk, NY: IBM Corp.

Jadalla, A., \& Lee, J. (2012). The relationship between acculturation and general health of Arab Americans. Journal of Transcultural Nursing, 23(2), 159-165. doi:

$10.1177 / 1043659611434058$

Jadalla, A., \& Lee, J. (2015). Validation of Arabic and English versions of the ARSMA-II acculturation rating scale. Journal of Immigrant and Minority Health, 17(1), 208-216. doi: $10.1007 / \mathrm{s} 10903-013-9889-2$

Jasperse, M., Ward, C., \& Jose, P. E. (2012). Identity, perceived religious discrimination, and psychological well-being in Muslim immigrant women. Applied Psychology, 61(2), 250271. doi: 10.1111/j.1464-0597.2011.00467.x

Jones, P. S., Lee, J. W., Phillips, L. R., Zhang, X. E., \& Jaceldo, K. B. (2001). An adaptation of Brislin's translation model for cross-cultural research. Nursing Research, 50(5), 300304. doi: 10.1097/00006199-200109000-00008 


\section{BARRIERS AND FACILITATORS OF SEEKING PSYCHOLOGICAL HELP}

Kawika Allen, G. E., Wang, K. T., Richards, P. S., Ming, M., \& Suh, H. N. (2018). Religious discrimination scale: Development and initial psychometric evaluation. Journal of Religion and Health. Advance online publication. doi: 10.1007/s10943-018-0617-z

Kelly Jr, E. W., Aridi, A., \& Bakhtiar, L. (1996). Muslims in the United States: An exploratory study of universal and mental health values. Counseling and Values, 40(3), 206-218. doi: 10.1002/j.2161-007X.1996.tb00853.X

Kenny, D. A. (2015, November 24). Measuring model fit. Retrieved from http://davidakenny.net/cm/fit.htm

Khan, Z. (2006). Attitudes toward counseling and alternative support among Muslims in Toledo, Ohio. Journal of Muslim Mental Health, 1(1), 21-42. doi: $10.1080 / 15564900600654278$

Kline, R. B. (2005). Principles and practice of structural equation modeling (2nd ed.). New York, NY: Guilford Press.

Komiya, N., Good, G. E., \& Sherrod, N. B. (2000). Emotional openness as a predictor of college students' attitudes toward seeking psychological help. Journal of Counseling Psychology, 47(1), 138-143. doi: 10.1037/0022-0167.47.1.138

Kwok, J., Atencio, J., Ullah, J., Crupi, R., Chen, D., Roth, A. R., .. \& Brondolo, E. (2011). The Perceived Ethnic Discrimination Questionnaire - Community Version: Validation in a multiethnic Asian sample. Cultural Diversity and Ethnic Minority Psychology, 17(3), 271-282. doi: 10.1037/a0024034

Lee, S., Nguyen, H. A., \& Tsui, J. (2011). Interview language: a proxy measure for acculturation among Asian Americans in a population-based survey. Journal of Immigrant and Minority Health, 13(2), 244-252. doi: 10.1007/s10903-009-9278-z 


\section{BARRIERS AND FACILITATORS OF SEEKING PSYCHOLOGICAL HELP}

Liptak, A., \& Shear, M. D. (2018). Trump's travel ban is upheld by Supreme Court. New York Times, p. A1. https://www.nytimes.com/2018/06/26/us/politics/supreme-court-trump$\underline{\text { travel-ban.html }}$

Martin, M. B. (2015). Perceived discrimination of Muslims in health care. Journal of Muslim Mental Health, 9(2), 41-69. doi: 10.3998/jmmh.10381607.0009.203

MacCallum, R. C., Widaman, K. F., Zhang, S., \& Hong, S. (1999). Sample size in factor analysis. Psychological Methods, 4(1), 84. doi: 1082-989X/99/S3.00

MacCallum, R. C., Widaman, K. F., Preacher, K. J., \& Hong, S. (2001). Sample size in factor analysis: The role of model error. Multivariate Behavioral Research, 36(4), 611-637. doi: 10.1207/S15327906MBR3604_06

McNeilly, M. D., Anderson, N. B., Armstead, C. A., Clark, R., Corbett, M., Robinson, E. L., .. \& Lepisto, E. M. (1996). The perceived racism scale: a multidimensional assessment of the experience of white racism among African Americans. Ethnicity \& Disease, 6(12), 154-166. Retrieved from: https://www.ethndis.org/edonline/index.php/ethndis

Mohamed, B. (2018). New estimates show U.S. Muslim population continues to grow. Retrieved from: http://www.pewresearch.org/fact-tank/2018/01/03/new-estimates-showu-s-muslim-population-continues-to-grow/

Nadal, K. L., Griffin, K. E., Hamit, S., Leon, J., Tobio, M., and Rivera, D. P. (2012). Subtle and overt forms of Islamophobia: Microaggressions toward Muslim Americans. Journal of Muslim Mental Health, 6(2), 15-37. doi: 10.3998/jmmh.10381607.0006.203

Padela, A. I., Killawi, A., Forman, J., DeMonner, S., \& Heisler, M. (2012). American Muslim perceptions of healing: key agents in healing, and their roles. Qualitative Health Research, 22(6), 846-858. doi: 10.1177/1049732312438969 


\section{BARRIERS AND FACILITATORS OF SEEKING PSYCHOLOGICAL HELP}

Padela, A. I., \& Heisler, M. (2010). The association of perceived abuse and discrimination after September 11, 2001, with psychological distress, level of happiness, and health status among Arab Americans. American Journal of Public Health, 100(2), 284-291. doi: 10.2105/AJPH.2009.164954

Pew Research Center on Religion and Public Life. (2018). Muslims in America: Immigrants and those born in U.S. see life differently in many ways. Retrieved from: http://www.pewforum.org/essay/muslims-in-america-immigrants-and-those-born-in-u-s$\underline{\text { see-life-differently-in-many-ways/ }}$

Read, J. (2008). Muslims in America. Contexts, 7(4), 39-43. doi: 10.1525/ctx.2008.7.4.39

Rippy, A. E., \& Newman, E. (2006). Perceived religious discrimination and its relationship to anxiety and paranoia among Muslim Americans. Journal of Muslim Mental Health, 1, 520. doi: 10.1080/15564900600654351

Ryan, L. (2011). Muslim women negotiating collective stigmatization: 'We're just normal people'. Sociology, 45(6), 1045-1060. doi: 10.1177/0038038511416170

StataCorp. (2017). Stata Statistical Software: Release 15. College Station, TX: StataCorp LLC.

Tabassum, R., Macaskill, A., \& Ahmad, I. (2000). Attitudes towards mental health in an urban Pakistani community in the United Kingdom. International Journal of Social Psychiatry, 46(3), 170-181. doi: 10.1177/002076400004600303

Thomson, M. D., \& Hoffman-Goetz, L. (2009). Defining and measuring acculturation: a systematic review of public health studies with Hispanic populations in the United States. Social Science \& Medicine, 69(7), 983-991. doi: 10.1016/j.socscimed.2009.05.011 


\section{BARRIERS AND FACILITATORS OF SEEKING PSYCHOLOGICAL HELP}

United States Department of State. (2009). Being Muslim in America. Washington D.C.: Bureau of International Information on Programs. Retrieved from: http://www.america.gov/publications/books/being-muslim-in-america.html

Vogel, D. L., Armstrong, P. I., Tsai, P., Wade, N. G., Hammer, J. H., Efstathiou, G., \& ... Topkaya, N. (2013). Cross-cultural validity of the Self-Stigma of Seeking Help (SSOSH) scale: Examination across six nations. Journal of Counseling Psychology, 60(2), 303-310. doi:10.1037/a0032055

Vogel, D. L., Strass, H. A., Heath, P. J., Al-Darmaki, F. R., Armstrong, P. I., Baptista, M. N., ... \& Mackenzie, C. S. (2017). Stigma of seeking psychological services: Examining college students across ten countries/regions. The Counseling Psychologist, 45(2), 170192. doi:10.1177/0011000016671411

Vogel, D. L., Wade, N. G., \& Haake, S. (2006). Measuring the self-stigma associated with seeking psychological help. Journal of Counseling Psychology, 53(3), 325-337. doi:10.1037/0022-0167.53.3.325

Vogel, D. L., Wade, N., \& Hackler, A. (2007). Perceived public stigma and the willingness to seek counseling: The mediating roles of self-stigma and attitudes towards counseling. Journal of Counseling Psychology, 54(1), 40-50. doi: 10.1037/0022-0167.54.1.40

Vogel, D. L., Wester, S. R. (2003). To seek help or not to seek help: The risks of selfdisclosure. Journal of Counseling Psychology, 50(3), 351-361. doi: 10.1037/00220167.50.3.351

Whiteoak, J. W., Crawford, N. G., \& Mapstone, R. H. (2006). Impact of gender and generational differences in work values and attitudes in an Arab culture. Thunderbird International Business Review, 48(1), 77-91. doi:10.1002/tie.20086 
BARRIERS AND FACILITATORS OF SEEKING PSYCHOLOGICAL HELP

Youssef, J., \& Deane, F. P. (2006). Factors influencing mental-health help-seeking in Arabicspeaking communities in Sydney, Australia. Mental Health, Religion \& Culture, 9(1), 43-66. doi: 10.1080/13674670512331335686 
BARRIERS AND FACILITATORS OF SEEKING PSYCHOLOGICAL HELP

\section{APPENDICES}


BARRIERS AND FACILITATORS OF SEEKING PSYCHOLOGICAL HELP

\section{Appendix A: Self-Stigma of Seeking Help Scale}

(SSOSH; Vogel et al., 2006)

Indicate for the statement below the degree to which the change reflected in the question is true in your life as a result of your parents' divorce, using the following scale:

$1=$ Strongly Disagree; 2 = Disagree; $3=$ Neutral; $4=$ Agree $; 5=$ Strongly Agree

1. I would feel inadequate if I went to a therapist for psychological help.

2. My self-confidence would NOT be threatened if I sought professional help.

3. Seeking psychological help would make me feel less intelligent.

4. My self-esteem would increase if I talked to a therapist.

5. My view of myself would not change just because I made the choice to see a therapist.

6. It would make me feel inferior to ask a therapist for help.

7. I would feel okay about myself if I made the choice to seek professional help.

8. If I went to a therapist, I would be less satisfied with myself.

9. My self-confidence would remain the same if I sought help for a problem I could not solve.

10. I would feel worse about myself if I could not solve my own problems. 


\section{Appendix B: Acculturation Rating Scale for Arabic Americans-II - Attraction to American}

\section{Culture Subscale}

(AAmC; Jadalla \& Lee, 2015)

Please rate the extent to which the following statements describe you using the following scale:

$1=$ Not like me; $2=$ Somewhat unlike me; $3=$ Neutral $; 4=$ Somewhat like me $5=$ A lot like me

1. I enjoy listening to English language music

2. I speak English

3. I enjoy English language movies (American movies)

4. I associate with White Americans

5. I enjoy English language TV (American TV)

6. My thinking is done in English

7. I write in English

8. I enjoy reading in English

9. My contact with the USA has been (none $\rightarrow$ almost always)

10. My friends now are of White origin

11. My friends, while I was growing up, were of American origin

12. I like to identify myself as an American

13. I like to identify myself as a White American 
BARRIERS AND FACILITATORS OF SEEKING PSYCHOLOGICAL HELP

\section{Appendix C: Short Muslim Practice and Belief Scale}

(S-MPBS; AlMarri et al., 2009)

Please rate the extent to which you agree with the following statements using the scale below:

5 = Strongly Disagree; 4 = Disagree; $3=$ Neutral; $2=$ Agree $; 1=$ Strongly Agree

1. All Muslim countries should be governed by absolute Shariah law.

2. Religion should govern all my actions.

3. If someone commits a crime they should be punished according to absolute Shariah law (e.g., stoning to death for adultery).

4. Men/women should not shake hands with opposite sex in public.

5. The portrayal (picture, painting) of a human figure (such as a face) should not be allowed.

Please rate the extent to which you engage in the following behaviors using the scale below:

1 = I never do this; 2 = I rarely do this; 3 = I sometimes do this; $4=$ I very often do this; 5

$=$ I always do this

6. I follow all the pillars of Islam.

7. I pray five times day.

8. I read the Qur'an.

9. I fast the month of Ramadan. 
Appendix D: Brief Perceived Ethnic Discrimination Questionnaire - Community Version

\section{Lifetime Exposure Scale}

(PEDQ-CV; Brondolo et al., 2005).

Think about your ethnicity/race. What group do you belong to? Do you think of yourself as: Asian? Black? Latino? White? Native American? American? Caribbean? Irish? Italian? Korean? Arab? Another group?

\section{YOUR ETHNICITY/RACE:}

How often have any of the things listed below happened to you, because of your ethnicity?

\section{BECAUSE OF YOUR ETHNICITY/RACE ...}

How often... Never Sometimes

1. Have you been treated unfairly by teachers, principals, or other staff at school?

$\begin{array}{lllll}1 & 2 & 3 & 4 & 5\end{array}$

2. Have others thought you couldn't do things or handle a job?

$\begin{array}{lllll}1 & 2 & 3 & 4 & 5\end{array}$

3. Have others threatened to hurt you (ex: said they would hit you)?

$\begin{array}{lllll}1 & 2 & 3 & 4 & 5\end{array}$

4. Have others actually hurt you or tried to hurt you (ex: kicked or hit you)?

$\begin{array}{lllll}1 & 2 & 3 & 4 & 5\end{array}$

5. Have policemen or security officers been unfair to you?1

$\begin{array}{llll}2 & 3 & 4 & 5\end{array}$

6. Have others threatened to damage your property?

7. Have others actually damaged your property?

1

8. Have others made you feel like an outsider who

1

2

3
doesn't fit in because of your dress, speech, or other characteristics related to your ethnicity?

9. Have you been treated unfairly by co-workers

(n)


BARRIERS AND FACILITATORS OF SEEKING PSYCHOLOGICAL HELP

or classmates?

\section{BECAUSE OF YOUR ETHNICITY/RACE ...}

How often...

\begin{tabular}{|c|c|c|}
\hline Never & Sometimes & $\begin{array}{l}\text { Very } \\
\text { Often }\end{array}$ \\
\hline 1 & 3 & 5 \\
\hline
\end{tabular}

10. Have others hinted that you are dishonest or can't be trusted?

11. Have people been nice to you to your face, but said bad things about you behind your back?

$\begin{array}{lllll}1 & 2 & 3 & 4 & 5\end{array}$

12. Have people who speak a different language made you feel like an outsider?

13. Have others ignored you or not paid attention to you?

14. Has your boss or supervisor been unfair to you?

15. Have others hinted that you must not be clean?

16. Have people not trusted you?

1

2

3

4

5

17. Has it been hinted that you must be lazy?

1

2

3

The subscales are as follows:

Exclusion: 8,11,12,13

Workplace discrimination: $1,2,9,14$

Stigmatization: 5, 10, 15, 16, 17

Threat and harassment: 3,4,6,7 


\section{Appendix E: Religious Discrimination Scale}

(RDS; Kawika Allen et al., 2018)

Please rate how often during your life you have had the following experiences in the United

States:

$1=$ never 2 = rarely 3 = sometimes 4 = frequently; 5 = always

1. I felt disrespected because of my religious views

2. I was ignored because I am a religious person

3. People assumed things about me because of my religion.

4. I felt inclined to keep my religious affiliation private.

5. I was afraid of others finding out about my religious beliefs.

6. I have felt socially avoided by others due to my religion

7. I was passed over for opportunities due to my religion

8. I sense hostility from others because of my religious affiliation.

9. I have heard people make unfriendly remarks about my religion.

10. Others hold negative stereotypes of people with my religion.

11. I do not feel free to express who I am religiously

$\underline{\text { Subscale Items }}$

Perceived Prejudice: 8, 7, 6, 2, 1

Closet Symptoms: 4, 5, 11

Negative labels: 10, 9, 3 
BARRIERS AND FACILITATORS OF SEEKING PSYCHOLOGICAL HELP

\section{Appendix F: Demographic Questionnaire}

1. What is your age in years?

2. What is the highest degree or level of school you have completed or are currently working on?

- No schooling completed

- Nursery school to 8th grade

- Some high school, no diploma

- High school graduate, diploma or the equivalent (for example: GED)

- Some college credit, no degree

- Trade/technical/vocational training

- Associate degree

- Bachelor's degree

- Master's degree

- Professional degree

- Doctorate degree

3. Please select your relationship status below:

- Single, never married

- In a relationship

- Engaged

- Married or domestic partnership

- Widowed

- Divorced

- Separated 
4. What was your total household income before taxes during the past 12 months?

- Less than $\$ 25,000$

- $\$ 25,000$ to $\$ 34,999$

- $\$ 35,000$ to $\$ 49,999$

- $\$ 50,000$ to $\$ 74,999$

- $\$ 75,000$ to $\$ 99,999$

- $\$ 100,000$ to $\$ 149,999$

- $\$ 150,000$ or more

5. What is your gender?

- Male

- Female

- Self-Identify As:

6. Were you born in the United States or a U.S. territory?

- Yes

- No

7. How long have you lived in the United States?

- less than 3 months

- 3 months -1 year

- 2-5 years

- 6-10 years

- 11-15 years

- 16-20 years

- over 21 years 
- All of my life

8. What is your generational status in the United States?

- In U.S. for specific purpose (i.e. school/work) with plans to return to native country

- $1^{\text {st }}$ generation (i.e. born outside of U.S. or to parents who were not U.S. citizens)

- $2^{\text {nd }}$ generation (i.e. born in the U.S. with at least one $1^{\text {st }}$ generation parent)

- $3^{\text {rd }}$ generation or higher (i.e. born in the U.S. with both parents born in the U.S. or U.S. territories)

9. Is English your first/primary language?

- Yes

- No

- If no, what is your first/primary language?

10. What is your ethnicity?

- African American/Black

- Asian/Pacific Islander

- Caucasian/White

- Hispanic or Latino

- Indigenous or Aboriginal Person

- Middle Eastern or North African

- Multiracial

- Other:

11. What region best describes your country of origin?

- Asia

- Caribbean 
- Central America

- Europe

- Middle East/North Africa

- Sub-Saharan Africa

- North America

- Oceana

- South American

- Other:

12. What best describes your religious identity?

- Sunni

- Shi’a

- Ahmadiyya

- Ibadi

- Sufism

- Self-Identify As:

13. Please choose your $1^{\text {st }}, 2^{\text {nd }}$, and $3^{\text {rd }}$ choices of help for mental health or psychological problems:

- Doctor

- family member(s)

- Religious Leader(s)

- Mental Health Worker

- Close Friend(s)

- No One 
BARRIERS AND FACILITATORS OF SEEKING PSYCHOLOGICAL HELP

14. Have you ever sought professional help for:

- Physical concerns

$\begin{array}{ll}\circ & \text { Never } \\ \circ & 1-2 x \\ \circ & 3-5 x \\ \circ & \text { More than } 5 x\end{array}$

- Mental health concerns
○ Never
$\circ 1-2 x$
$\circ 3-5 x$
$\circ$ More than $5 \mathrm{x}$ 


\section{Appendix G: Cover Letter}

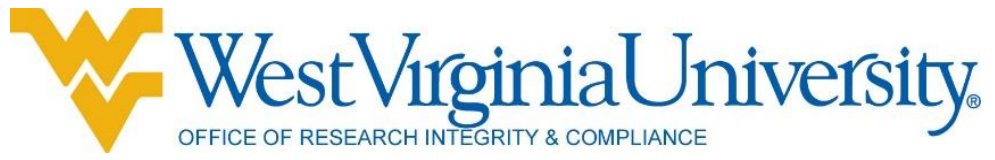

Do you want to participate in a research project to explore factors related to barriers and facilitators of seeking mental health services for Muslims living in the United States?

To participate in this study, you must:

- Be 18 years of age or older

- Identify as Muslim

- Have lived in the United States for 3 months or more

- Read English

If you meet the above criteria, your thoughts about this topic are wanted for a research study! This project is being conducted by Sarah Milam, M.A. in the Department of Counseling Psychology at West Virginia University (WVU), as part of my Ph.D. in Counseling Psychology. This project is being supervised by Dr. Lisa Platt, an assistant professor in the Department of Counseling Psychology at WVU. Your participation in this project is greatly appreciated and will take approximately 15 minutes to fill out this online questionnaire.

Your involvement in this project will be kept as confidential as legally possible and you will not be asked to disclose any identifying information that will be linked to your data (i.e. name, email address, etc.). All data will be reported in the aggregate.

I will not ask any information that should lead back to your identity as a participant. Your participation is completely voluntary. You may skip any question that you do not wish to answer and you may discontinue at any time. West Virginia University's Institutional Review Board has acknowledgement and approval of this project on file.

I hope that you will participate in this research project, as it could be beneficial in understanding factors related to mental health care for Muslims living in the United States. In addition, you will have the chance to enter a drawing to win one of 10 available \$25 Amazon gift cards for completing this survey! At the end of this survey you will have the option to click a link to open a separate survey to enter the email address you would like the gift card sent to. Please note, for your privacy, email addresses are not linked with your responses on this survey.

Please only complete the survey one time and only complete it if you meet the criteria listed above. Respondents whose email addresses are flagged as fraudulent or duplicate responders will be disqualified from the gift-card selection pool. Thank you very much for your time. Should you have any questions about this letter or the research project, please feel free to contact Sarah Milam at srberkey@mail.wvu.edu. 
BARRIERS AND FACILITATORS OF SEEKING PSYCHOLOGICAL HELP

If you are interested in completing this survey, please click the red arrow at the bottom of the page to begin.

Thank you for your time and help with this project.

Sincerely,

Sarah Milam, M.A. 


\section{Appendix H: Informed Consent Form}

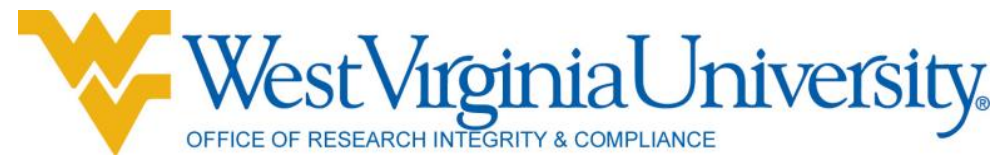

Human Research Protocol

Only Minimal Risk Consent Form

Without HIPAA

\section{Only Minimal Risk}

Consent Information Form (without HIPAA)

Principal Investigator

Department

Protocol Number

Study Title

United States

Co-Investigator(s)

Sponsor (if any)
Lisa Platt, Ph.D.

Counseling, Rehabilitation, Counseling, and Counseling Psychology

Click here to enter text.

Barriers and Facilitators of Seeking Psychological Help for Muslims in the

Sarah Milam, M.A.

Click here to enter text.

\section{Contact Persons}

If you have any questions, concerns, or complaints about this research, you can contact Dr. Lisa Platt at Lisa.Platt@mail.wvu.edu or Sarah Milam, M.A. at srberkey@ mail.wvu.edu.

For information regarding your rights as a research subject, to discuss problems, concerns, or suggestions related to the research, to obtain information or offer input about the research, contact the Office of Research Integrity and Compliance at (304) 293-7073.

In addition if you would like to discuss problems, concerns, have suggestions related to research, or would like to offer input about the research, contact the Office of Research Integrity and Compliance at 304-293-7073.

\section{Introduction}

You have been asked to participate in this research study. This study is being conducted by Sarah Milam, M.A. in the Department of Counseling, Rehabilitation, Counseling, and Counseling Psychology at West Virginia University.

\section{Purpose(s) of the Study}

The purpose of this study is to learn more about factors related to mental health help-seeking beliefs for a diverse group of Muslims living in the United States.

\section{Description of Procedures}

This study involves completing an anonymous online survey and will take approximately 15 minutes for you to complete. You will be asked to fill out a questionnaire regarding experiences of discrimination, acculturation, religious beliefs, and mental-health care beliefs. You do not have to answer all the questions and can discontinue the survey at any time.

\section{Discomforts}

There are no known or expected risks from participating in this study, except for the mild frustration associated with answering the questions.

\section{Benefits}

You may not receive any direct benefit from this study. The knowledge gained from this study may eventually benefit others.

\section{Financial Considerations}




\section{BARRIERS AND FACILITATORS OF SEEKING PSYCHOLOGICAL HELP}

There are no special fees for participating in this study. At the completion of the survey, you will have the option to click a link to enter your email on a separate survey (not attached to your original response data) in order to be compensated with a five dollar Amazon gift card for your participation.

\section{Confidentiality}

Any information about you that is obtained as a result of your participation in this research will be kept as confidential as legally possible. Your survey responses will not be connected with your email address if you choose to provide it to receive a gift card compensation, as your email will be entered into a separate survey from your main study responses. These research records, just like hospital records, may be subpoenaed by court order or may be inspected by the study sponsor or federal regulatory authorities (including the FDA if applicable) without your additional consent; however, all data will be collected anonymously and your identity will not be connected to your survey responses.

In addition, there are certain instances where the researcher is legally required to give information to the appropriate authorities. These would include mandatory reporting of infectious diseases, mandatory reporting of information about behavior that is imminently dangerous to your child or to others, such as suicide, child abuse, etc.

In any publications that result from this research, neither your name nor any information from which you might be identified will be published without your consent.

\section{Voluntary Participation}

Participation in this study is voluntary. You are free to withdraw your consent to participate in this study at any time.

Refusal to participate or withdrawal will involve no penalty to you.

In the event new information becomes available that may affect your willingness to participate in this study, this information will be given to you so that you can make an informed decision about whether or not to continue your participation.

You have been given the opportunity to ask questions about the research, and you have received answers concerning areas you did not understand.

I willingly agree to be in the study.
$\circ$ Yes
$\circ$ No

Phone: 304-293-7073

Fax: 304-293-3098

http://oric.research.wvu.edu

Chestnut Ridge
Research
Building
886 Chestnut
Ridge Road
PO Box 6845
Morgantown,
WV 26506-6845




\section{Appendix I: Institutional Review Board Approval}

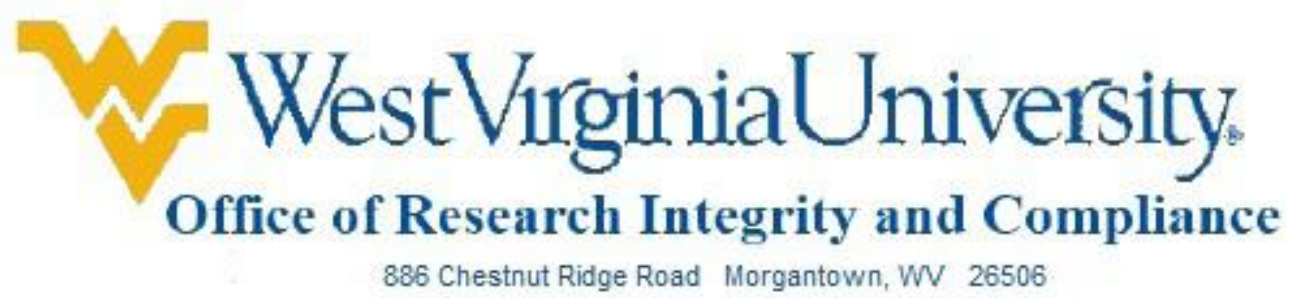

\section{Acknowledgement of Exemption}

$12 / 06 / 2018$

To: Lisa Platt

From: WVU Office of Research Integrity \& Compliance

Protocol Type: Exempt Approval Date: 12/06/2018

Submission Type: Initial Expiration Date: 12/05/2021

Funding: N/A

WVU Protocol \#: 1811351490

Protocol Title: Barriers and Facilitators of Seeking Psychological Help for Muslims Living in the United States

The West Virginia University Institutional Review Board has reviewed your submission of Exempt protocol 1811351490. Additional details regarding the review are below:

- Sarah Milam needs to link CITI training with KC by logging in to CITI by clicking on LOG IN THROUGH MY INSTITUTION.

- This research study was granted an exemption because the Research involves educational tests, survey procedures, interview procedures or observation of public behavior and (i) information obtained is recorded in such a manner that human subjects cannot be identified, directly or through identifiers linked to the subjects; and (ii) any disclosure of the human subjects responses outside the research could not reasonably place the subjects at risk of criminal or civil liability or be damaging to the subjects financial standing, employability, or reputation [45 CFR 46.101(2)]. All exemptions are only good for three years. If this research extends more than three years beyond the approved date, then the researcher will have to request another exemption. The following documents have been acknowledged for use in this study and are available in the WVU+kc system:

The following documents were reviewed and approved for use as part of this submission. Only the documents listed below may be used in the research. Please access and print the files in the Notes \& Attachments section of your approved protocol.

- Recruitment Statements and Materials.pdf

- Milam Qualtrics Main Survey_For IRB 12.5.18.pdf

- Milam Qualtrics Email Request Survey_For IRB 11.28.18.pdf

WVU IRB acknowledgement of protocol 1811351490 will expire on 12/05/2021. 
If the study is to continue beyond the expiration date, a renewal application must be submitted no later than two (2) weeks prior to expiration date. It is your responsibility to submit your protocol for renewal.

Once you begin your human subjects research, the following regulations apply:

1. Unanticipated or serious adverse events and/or side effects encountered in this research study must be reported to the IRB within five (5) days, using the Notify IRB action in the electronic protocol.

2. Any modifications to the study protocol should be submitted only if there will be an increase in risk to subjects accompanying the proposed change(s).

3. You may not use a modified information sheet until it has been reviewed and acknowledged by the WVU IRB prior to implementation.

The Office of Research Integrity and Compliance will be glad to provide assistance to you throughout the research process. Please feel free to contact us by phone, at 304.293.7073 or by email at IRB@mail.wvu.edu.

Sincerely,

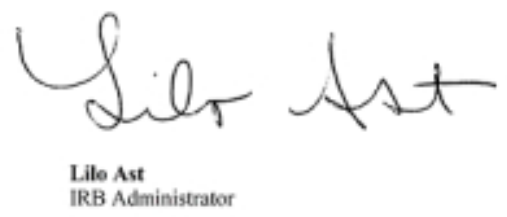


CURRICULUM VITAE

\author{
Sarah R. B. Milam, M.A. \\ 547 Hill Street, Apt A, Morgantown, WV 26505 \\ Phone: 443-618-8589 \\ E-mail: srberkey@mix.wvu.edu
}

EDUCATION

Ph.D. in Counseling Psychology

August 2020

West Virginia University, Morgantown, WV

(Anticipated)

GPA: 4.0

Dissertation: Barriers and facilitators of seeking psychological help for Muslims living in the United States

APA Accredited Pre-Doctoral Psychology Internship

July 2020

Carruth Center for Psychological and Psychiatric Services

West Virginia University, Morgantown, WV

(Anticipated)

Master of Arts in Counseling Psychology

May 2015

Towson University, Towson, MD

GPA: 4.0

Thesis: A mixed methods investigation of posttraumatic growth in young adults following parental divorce

Bachelor of Arts in Psychology \& Government \& Politic University of Maryland, College Park, MD

May 2011

GPA: 3.7

CLINICAL

\& RELATED EXPERIENCE
APA Accredited Psychology Internship

Carruth Center for Psychological and Psychiatric Services; Morgantown, WV

West Virginia University Licensed Supervisors: Narayan Gold, Ph.D.; T. Anne Hawkins, Ph.D.

Duties: Provide individual, group, and crisis counseling; conduct LD/ADHD assessments, write integrated reports, and provide feedback and treatment plan to clients; conduct individual supervision of advanced doctoral students; facilitate therapy groups; provide outreach; Act as liaison and consultant to Adventure WV and residence halls and facilitate collaborative programming to facilitate student wellness; contribute to weekly case conferences and staff meetings; member of Diversity Committee and Training Committee.

World Scout Jamboree Doctoral Clinician July 2019

World Organization of the Scout Movement; Mount Hope, WV

Licensed Supervisor: Daniel McNeil, Ph.D.

Duties: Provided crisis and supportive counseling, mental health evaluations, case management to diverse international adult staff and youth scouts who attended the 2019 World Scout Jamboree in West Virginia. Worked in integrated care setting alongside medical doctors and nurses who provided referrals and consultations. Presenting concerns included: bipolar disorder, sexual assault, adjustment concerns, multicultural concerns, conflict management, anxiety, depression, and suicidality. 


\section{BARRIERS AND FACILITATORS OF SEEKING PSYCHOLOGICAL HELP}

Carruth Center for Psychological and Psychiatric Services; Morgantown, WV

West Virginia University

Licensed Supervisors: Tandy McClung, Ed.D.; Ashley Kasardo, Psy.D.; Narayan Gold,

Ph.D.; Daniel Long II, Ph.D.; Christine Simpson, Psy.D.

Duties: Provide brief and long-term individual therapy; conduct ADHD/LD assessments and write integrated reports; conduct crisis appointments, triage intakes and consultations; and develop and implement outreach activities for diverse college students at West Virginia University. Prepare and lead women's interpersonal process group and drop-in psychoeducational group. Participate in individual and group supervision by licensed psychologists.

\section{Advanced Doctoral Practicum Clinician}

Monongalia Child Advocacy Center; Morgantown, WV

August 2016-May 2018

Licensed Supervisor: Laura Capage, Ph.D.

Duties: Provided individual and group therapy to children who have been abused or neglected using a Trauma Focused-Cognitive Behavioral Therapy framework and principles from Parent Child Interaction Therapy. Collaborated with a multidisciplinary team of parents and other care-givers, school professionals, Child Protective Services, and the court system to provide care to clients. Engaged in crisis intervention sessions. Developed and implemented a children's therapy group and provided supervision to other training clinicians co-leading the group. Received individual and group supervision by licensed psychologist.

\section{Master's Practicum Clinician}

August 2013- May 2014

Key Point Health Services; Catonsville, $M D$

Supervisor: Linda Wilkens, MA, LCPC

Duties: Provided individual therapy to twelve clients per week with broad ranging concerns including anxiety and depression, bipolar disorder, thought disorders, and domestic violence. Consulted with psychiatrists regarding client mental health. Participated in weekly individual and group supervision.

Group Facilitator/Service Learning Leader

Family Crisis Center of Prince George's County, MD

Supervisor: Karen O'Brien, Ph.D.

Duties: Developed and ran therapeutic groups for children who experienced domestic violence (DV). Constructed group agendas focusing on physical health, enhancing selfesteem, and positive release of emotions. Participated in group supervision and discussion.

Group Leader

Student Support Network; Towson University, Towson, MD

August 2013 - December 2013

Supervisor: Marianne Dunn, Ph.D.

Duties: Developed and facilitated 7 psycho-educational and peer advocate groups for 13 undergraduates. Trained students in awareness, helping skills, and referral resources for mental health topics such as core supportive skills, depression, anxiety, suicide, and assault.

\section{Peer Counselor}

Help Center Hotline; University of Maryland, College Park, MD

September 2007 - May 2008

Duties: Participated in trainings and answered phone calls on peer counseling and crisis intervention hotline.

\section{CLINCAL} OUTREACH INITIATIVES
Student Wellness Retreat

West Virginia University; Morgantown, WV
October 2019 
Collaborated with Campus Recreation, Carruth Center, Adventure WV, and campus nutritionist to engage students in a 6-hour wellness retreat in which they engaged in teambuilding and social support activities, healthy eating and discussion with campus nutritionist, outdoor yoga, personal reflection, goal setting, and small group discussion.

Using Nature to Promote Self-Care

Spring 2019, Fall 2019

West Virginia University; Morgantown, WV

Developed and led 4 outdoor experimental workshops in collaboration with WVU's Outdoor Recreation Center to promote understanding of use of outdoor resources, mindfulness, and social connection to promote coping, self-care, and wellness.

International Student Cultural Adjustment and Engagement

May, June 2019

West Virginia University; Morgantown, WV

Collaborated with 3 campus units to plan and lead 2 cultural adjustment workshops for international students which included going to a local forest for a hike, mindfulness activities, psychoeducation for coping skills, social engagement, and outreach.

International Student Engagement Workshop

Fall 2019

Collaborated with the Office of Global Affairs to provide team-building and social engagement programming for a group of 100 international students. Following the activities, students were informed of mental health services of campus and given resources to promote engagement.

International Student Stress Management Workshop

October 2018

West Virginia University; Morgantown, WV

Outreach workshop presented to international students focused on stress management skills and increasing accessibility of university counseling center services.

International Student Cultural Adjustment Workshop September 2018 West Virginia University; Morgantown, WV

Outreach workshop presented to international students focused on normalizing cultural adjustment difficulties and increasing accessibility of university counseling center services.

Growth Motivation and Positive Psychology Workshop March, 2018 West Virginia University; Morgantown, WV

Outreach workshop presented to 30 undergraduates to promote wellness at West Virginia University.

Balance and Time Management Workshop

West Virginia University; Morgantown, WV

January 2018

Outreach workshop presented to 30 undergraduates to promote wellness at West Virginia University.

The Use of Positive Psychology to Enhance Wellbeing Workshop

October 2017

West Virginia University; Morgantown, WV

Outreach workshop presented to 50 undergraduates to promote wellness at West Virginia

University.

Women's Giving Circle Presentation

April 2017

Morgantown, $W V$

Presented to over 50 women members of an all-female advocacy and donor group in

Morgantown, WV. Presentation provided information regarding the children's therapy group I developed and led at the Monongalia Child Advocacy Center funded by the WGC. 
Taught undergraduate students to promote healthy behaviors in their campus community through equipping them with skills to identify unhealthy behaviors across several domains including: alcohol and drug use, stress and sleep issues, sexual health, relationship and dating violence, and body image and nutrition. Students were empowered with knowledge of campus resources, referral information, and peer support skills. Engaged students in monthly supervision and support meetings.

Monongalia Child Advocacy Center Open House

January 2017 Morgantown, $W V$

Presented child advocacy support and referral information to community members including school counselors, county commissioner, and police officers in order to increase understanding of child abuse and resources for healing.

Out of the Darkness Suicide Prevention Walk and Fundraiser

April 2016

West Virginia University; Morgantown, WV

Organized team for WVU's first OOTD suicide prevention walk

Founding Member \& Public Relations Coordinator

National Alliance on Mental Illness; Towson University, Towson, MD

July 2013 - July 2015

Established TU chapter by assisting in obtaining university sponsor, writing mission statement and member guidelines, and recruitment. Publicized programming related to raising awareness and reducing mental health stigma on campus and in greater Baltimore area.

Program Coordinator

June 2013 - December 2013

Student Support Network; Towson University, Towson, MD

Developed and implemented a Mental Health Advocate Training Program. Recruited over 90 undergraduates to apply. Constructed facilitator and participant training manuals for graduate Group Counseling students to lead psycho-educational groups. Managed program's online presence.

Graduate Mentor

January 2015 - June 2015

Maryland Psychological Association of Graduate Students; Towson, MD

Mentored undergraduate students interested in preparing for and attending graduate school.

PUBLICATIONS Milam, S. R. B., Schmidt, C. K. (2018). A mixed methods investigation of posttraumatic growth in young adults following parental divorce. The Family Journal, 26(2), 156-165. doi: $10.1177 / 1066480718781518$

Milam, S. R. B. (2017). Using self-forgiveness to address moral injury in combat veterans. Positive Psychology: Theory and Application, 13(1), 16-19.

Platt, L. F., Milam, S. R. B. (2017). Public discomfort with gender appearance-inconsistent bathroom use: The oppressive bind of Bathroom Laws for transgender individuals. Gender Issues, 35(3). 181-201. doi: 10.1007/s12147-017-9197-6

\section{SYMPOSIUMS/}

PRESENTATIONS Milam, S.R.B., (2018, August). Social justice implementation: A student's perspective. In Scheel, M.J. (Chair). Preparing psychology students for action as social justice advocates. Symposium conducted at the 2018 American Psychological Association Convention, San Francisco, CA.

Milam, S. R.B, Latorre, C. A., \& Darling, A. B., (2018, April). \#MeToo: Counseling psychologists addressing sexual misconduct. Roundtable conducted at the 2018 Great Lakes Regional Counseling Psychology Conference, Kalamazoo, MI. 
Thomas, K. A., Allen, T. S., \& Milam, S. R., (2018, April). Speak Out Reach Out: Student Led Social Justice and Service Organizations. Symposium conducted the 2018 Great Lakes Regional Counseling Psychology Conference, Kalamazoo, MI.

Cawthra, J., \& Milam, S. R. B. (2017, August). Magnifying the use of positive psychology: Using a mirror and megaphone in multiculturalism and self-care. Roundtable conducted at the 2017 American Psychological Association Convention, Washington, D.C.

Milam, S. R. B., (2017, August). Activating psychologists in doctoral level training. In P. DeLeon \& J. Sells (Chairs), Activating psychologists: Advocacy in science, education and practice. Symposium presented at the 2017 American Psychological Association Convention, Washington, D.C.

Platt, L.F, \& Milam, S. R. B., (2017, August). Public discomfort with gender appearanceinconsistent restroom use: The oppressive bind of HB-2. In N. Overstreet \& A. Bahns (Chairs), Data blitz: Using psychological research to address social issues. Symposium conducted at the 2017 American Psychological Association Convention, Washington, D.C.

Milam, S. R. B. \& Darling, A. B. (2017, April). Clinical implications for counseling psychologists. In C. Fais (Chair), Recognizing racial trauma: Research, clinical implications, and advocacy work. Symposium presented at the 2017 Great Lakes Regional Counseling Psychology Conference, Muncie, IN.

Latorre, C., Milam S. R. B., Kinder, A., Hull, C., (2017, April). Get in on the action: Promoting social justice on campus. Roundtable conducted at the 2017 Great Lakes Regional Counseling Psychology Conference, Muncie, IN.

Milam, S. R. B., Keutzer, B., Thomas, K., (2017 February). Speak Out Reach Out: Advocating for social justice through grassroots leadership. Roundtable conducted at 2017 WVU Graduate and Professional Student Leadership Conference, Morgantown, WV.

Milam, S. R. B., \& Latorre, C., (2016, April). Implications and suggestions for future research. In S. R. B. Milam (Chair), Forgiveness and hope as pathways to decrease prejudice and increase inclusivity in a multicultural society following terrorism. Symposium presented at the 2016 Great Lakes Regional Counseling Psychology Conference, Bloomington, IN.

Milam, S. R. B., \& Greenbaum, H. (2016, April). Advocacy implications for counseling psychologists. In J. L. Shaw (Chair), Demystifying human trafficking in the United States. Symposium presented at the 2016 Great Lakes Regional Counseling Psychology Conference, Bloomington, IN.

Scott, 0., Shaw, J., \& Milam, S. R. B. (2016, April). Speaking out for Syrian refugees: A creative appeal to the governor of Indiana. Social justice project presented at the 2016 Great Lakes Regional Counseling Psychology Conference, Bloomington, IN.

Ling, T., Gadson, C., Berkey, S. R., (2015, August). Increasing awareness of diversity in homogenous educational settings. In Keefe-Cooperman, K., (Chair), Transforming students in the classroom: Increasing diversity awareness. Symposium presented at the 2015 Annual Convention of the American Psychological Association, Toronto, Ontario, CA.

\section{POSTER}

PRESENTATIONS Shaw, J. L., Milam, S. R. B, Platt, L. F. (2019). Trustworthiness and stability in same and different sex relationships: Heterosexuals' attitudes toward bisexuality. Poster submitted to the 2020 Counseling Psychology Conference, New Orleans, LA. 
Milam, S. R. (2018, March). Inhibitory factors of seeking psychological help for Muslims of Arab-descent living in the U.S. Poster presented at the 2018 Muslim Mental Health Conference, Washington, D.C.

Darling, A. B., Swiger, T. A., \& Milam, S. R. (2018, April). Stigma and help-seeking among Arab Muslim college students in the U.S. Poster presented at the 2018 Great Lakes Regional Counseling Psychology Conference, Kalamazoo, MI.

Milam, S. R. (2018, April). Barriers and Facilitators of Seeking Psychological Help for Muslims of Arab Descent Living in U.S. Poster presented at the 2018 College of Education and Human Services Celebration of Scholars Conference, Morgantown, WV.

Milam, S. R. B. (2017, April). Self-forgiveness and posttraumatic growth in veterans suffering from moral injury. Poster presented at the 2017 Great Lakes Regional Counseling Psychology Conference, Muncie, IN.

Milam, S. R. B. (2016, October). Special considerations for career counseling with international students: Implications for research and practice. Poster presented at the 2016 West Virginia Psychological Association Fall Conference, Roanoke, West Virginia.

Taylor, J. T., Neimeyer, G. J., Latorre, C., Milam, S. R. B., (2016, August). Exploring professional development, cultural engagement, and competence with counseling psychologists. Poster prepared for the $124^{\text {th }}$ annual convention of the American Psychological Association, Denver, Colorado.

Taylor, J., Milam, S. R. B., \& Latorre, C. (2016, April). Incorporating social justice competence in the classroom: Tips, strategies, and experiential activities. Poster presented at the 2016 Great Lakes Regional Counseling Psychology Conference, Bloomington, IN.

Berkey, S. R., \& Schmidt, C. K. (2015, August). The relationship between social support, religion, and gender in posttraumatic growth following parental divorce. Poster presented at the 2015 American Psychological Association Convention, Toronto, Ontario, CA.

Weinberg, M., Berkey, S. R., \& Dunn, M. G., (2014, August). Student support network: evaluating a mental health advocacy and peer support training program. Poster presented at the 2014 American Psychological Association Convention, Washington DC.

Weinberg, M., Halstead, M., Berkey, S. R., Dunn, M. G., Schmidt, C. K., Keys, K., \& Lai, K., (2014, April). Evaluating the effectiveness of a mental health advocacy training program on a college campus. Poster presented at the 2014 Maryland Psychological Association of Graduate Students Convention, Columbia, MD.

\section{RESEARCH}

\section{EXPERIENCE Quantitative Dissertation}

May 2018-

present

Barriers and Facilitators for Seeking Psychological Help for Muslims living in the United States

West Virginia University, Morgantown, WV

Chair: Lisa Platt, Ph.D.

Conducting independent research using structural equation modeling to examine relationships between Islamic beliefs and practices, perceived religious discrimination, perceived ethnic discrimination, acculturation, and self-stigma of seeking psychological help in a sample of Muslims living in the United States. Proposal passed and in data collection phase. 
Public Discomfort with Gender Appearance-Inconsistent Restroom Use: The Oppressive Bind of "Bathroom Bills" for Gender Diverse Individuals

West Virginia University, Morgantown, WV

Faculty Advisor: Lisa Platt, $\mathrm{PhD}$

Assisted in development of hypothesis, analysis of data, and write-up of manuscript exploring public perceptions of bathroom usage based on gender appearance and the effect on transgender individuals.

Principle Investigator

September 2015-May 2016

Forgiveness and Hope as Pathways to Decrease Prejudice and Increase Inclusivity in a

Multicultural Society following Terrorism

West Virginia University, Morgantown, WV

Faculty Advisor: Jennifer Taylor, $\mathrm{PhD}$

Assembled research team of first year doctoral students to investigate the relationship between forgiveness and hope with Anti-Muslim prejudice and ethnocultural empathy. Literature review completed and in beginning stages of data collection.

\section{Research Assistant}

September 2015-August 2016

Law Enforcement Officers Killed and Assaulted

West Virginia University, Morgantown, WV

Faculty Advisor: Jeffery Daniels, $\mathrm{PhD}$

Trained in Consensual Qualitative Research (CQR) coding method. Acted as an auditor for transcripts previously coded using $\mathrm{CQR}$.

\section{Research Assistant}

October 2015-August 2016

Exploring Professional Development, Cultural Engagement, and Competence with

Counseling Psychologists

West Virginia University, Morgantown, WV

Faculty Advisor: Jennifer Taylor, PhD

Assisted with survey response collection and poster accepted to $124^{\text {th }}$ annual convention of the American Psychological Association.

Master's Thesis Research

September 2013-May 2015

The Relationship between Social Support, Religion, and Gender in Posttraumatic Growth (PTG) Following Parental Divorce

Towson University, Towson, MD

Faculty Advisor: Christa Schmidt, PhD

Proposed and conducted research based on empirical evidence and theory related to PTG.

Completed IRB proposal and developed plan for statistical analysis using SPSS. Proposal approved by committee following presentation and defense. Data collection, analysis, and final defense successfully completed.

\section{Research Assistant}

June 2013- August 2014

The Efficacy of the Student Support Network (SSN) Training Program at Towson University Towson University, Towson, MD

Faculty Advisor: Marianne Dunn, $\mathrm{PhD}$

Implemented and conducted research and program evaluation on the efficacy of the SSN at Towson University, a training program using psycho-educational groups to equip students to help peers in mental health crisis. Assisted in statistical analysis and presentation 


\section{BARRIERS AND FACILITATORS OF SEEKING PSYCHOLOGICAL HELP}

\section{Research Assistant}

2011

Study on Affective Transfer

University of Maryland, College Park, MD

Faculty Advisor: Catalina Kopetz, PhD and Anna Sheveland

Observed and coded clinical evaluations of men undergoing drug rehabilitation.

Ran participants, coded data, and interpreted results for study on affective transfer.

Research Assistant

Effects of Helping Skills Training on Interpersonal Development

University of Maryland, College Park

Faculty Advisor: Clara Hill, Ph.D. and Amber Farn

Coded responses using Consensual Qualitative Research method to questionnaire on effects of helping skills training on interpersonal development.

\section{Research Assistant}

January 2010-July 2010

Effects of Rejection on Relationship Strength

University of Maryland, College Park, MD

Faculty Advisor: Ryan Curtis, PhD

Collected data from participants in interactive mock-rejection scenario, performed data entry and participated in weekly discussions and problem solving.

\section{AD HOC}

REVIEWER FOR: 2020 APA Counseling Psychology Conference

APA Psych Shorts Science Video Contest, 2019

Positive Psychology: Theory and Application, 2018

Journal of Vocational Behavior, 2016

Counseling Psychology Quarterly, 2016

\section{TEACHING EXPERIENCE}

\section{RPTR 255 Wilderness Leadership in an International Context}

Co-Instructor, West Virginia University, New Zealand

May 2019

Organized, led, and taught 2.5-week study-abroad, 6-credit course for 10-students to New Zealand's South Island with a focus on theoretical and applied study of wilderness leadership in an international context. Course content included leadership theory, critical wilderness skills, international programming concepts, and environmental ethics from a social justice perspective.

WVU 191 Adventure WV First Year Seminar (3 sections) August 2018 - present Instructor, West Virginia University, Morgantown, WV

Engage students in experiential outdoor activities and grade students' reflection papers and summer orientation trip materials with a focus on academic adjustment and success.

RPTR 140A Sophomore Outdoor Adventure Reorientation January 2017-December 2017 Instructor, Trip Leader, West Virginia University, Morgantown, WV

Led and taught 19 WVU undergraduates on a 2.5-week outdoor experiential education 3credit course to Utah desert and backcountry. Taught curriculum based on positive psychology designed to provide them greater understanding of their personal strengths, leadership abilities, and direction regarding their major and career selection to increase their academic success. 
Taught academic class associated with incoming WVU Freshmen orientation trips. I equipped students with information and tools helpful for a successful transition from high school into college through facilitating critical thought and reflection on oneself, relationships, and place in society building off their summer outdoor field experience.

WVU 191 First Year Seminar (9 sections)

August 2015-June 2016

Instructor; West Virginia University, Morgantown, WV

Planned and taught course focused on developing college student critical thinking, metacognitive skill development, major and career exploration and planning, promotion of a global perspective, and awareness of campus resources. Individually coached students to facilitate greater academic achievement and success.

PSYC 605 Counseling Techniques

Teaching Assistant; Towson University, Towson, MD

September 2014 - December 2014

Supervisor: Shannon McClain, PhD

Graded assignments including transcripts and papers. Ran interactive lab to instruct students on how to employ basic counseling techniques. Observed and critiqued helping skills of graduate level helpers.

PSYC 470 Positive Psychology Seminar

November 2014

Guest Lecturer; Towson University, Towson, MD

Prepared and delivered lecture on posttraumatic growth. Facilitated discussion to understand the implications of PTG on clinical populations as well as in their own lives.

PSYC 420 Introduction to Counseling Psychology

August 2010 - December 2010

Teaching Assistant; University of Maryland, College Park, MD

Supervisor: Nashish Salahuddin, $\mathrm{PhD}$

Graded assignments and class papers. Developed case studies that integrate course

curriculum. Presented lecture on theory and research related to domestic violence.

\section{SOCIAL JUSTICE}

Serve as committee member aimed to promote the recruitment and retention of students and faculty from historically marginalized or underrepresented groups and to increase multicultural emphasis in WVU Counseling Psychology Program.

Experiential Learning Discussant leader

August 2017

Assisted in facilitating The Walk a Mile Experience at the 2017 American Psychological Association Convention, Washington, D.C. The activity focused on developing awareness and advocacy for families living in poverty in Maryland.

\section{Co-President \& Member}

May 2016 - August 2017, August 2015 - present Speak Out Reach Out; West Virginia University, Morgantown, WV

Organize programming and develop events to raise awareness of diversity and social justice at WVU and in surrounding community. Issues of focus include human sex trafficking, homelessness, employment equality, the Syrian refugee crisis, and immigration advocacy.

\section{Mental Wellbeing in the Job Search}

March 2016

Workshop presented at the Morgantown chapter meeting for Jobs for Life to assist disenfranchised community members experiencing difficulty while seeking employment.

Lobby Day, West Virginia Psychological Association September 2015 Participated with WV Psychological Association to lobby WV representatives for better mental health initiatives in WV public schools. 


\section{LEADERSHIP \& WORK EXPERIENCE}

\section{Student Representative}

APA Division 17 Section on Positive Psychology

Elected to lead and coordinate the Student Campus Representative Program. Facilitate recruitment of student members and assist with developing, gathering and disseminating resources and information to section members, select winners of section awards.

\section{Graduate Assistant to Orientation Trips Program}

Adventure $W V$; West Virginia University, Morgantown, WV

August 2016 - present and gear and food purchases; supervise student trip leaders; coordinate fall service trips, plan and lead outdoor leadership trips to Utah and New Zealand.

\section{Logistics Program Coordinator}

Adventure WV; West Virginia University, Morgantown, WV

April 2016 - August 2016

Supervised 8 student staff to prepare gear, food, and other trip logistics for WVU new student orientation trips for over 1,200 students. Engaged students in team building and train undergraduate trip leaders to assess and build inclusive group dynamics on outdoor orientation trips.

\section{Graduate Assistant}

August 2014 - May 2015

Human Resource Development MA Program; Towson University, Towson, MD Researched and compiled a database of Human Resource Development MA programs. Grade HRD Research course papers and assignments. Preform administrative duties.

\section{Graduate Assistant}

August 2012 - May 2014

Counseling Psychology MA Program; Towson University, Towson, MD

Provided administrative assistance to program director and faculty including writing and distributing program newsletter, grading class assignments, and preparing program applicant files and interviews. Assisted current and prospective students with program inquiries and course registration.

\section{Campus Staff}

August 2011 - August 2015

Cru - Campus Ministry Organization; University of Maryland, College Park, MD

Mentored college students through one on one relationships. Coordinated campus events, retreats, conferences, and fundraisers. Provided leadership to student executive teams.

\section{Behavioral Technician}

May 2011 - August 2011, May 2015 - August 2015 Anne Arundel Public Schools, Infants \& Toddlers Program; Glen Burnie, MD

Supervisor: Lynne Markowitz, M.A.Ed., Program Manager

Assisted development of fine motor skills and social interactions for 2-3 year olds with Autism and developmental delays.

Trip Leader and Challenge Course Facilitator

Outdoor Recreation Center; University of Maryland, College Park

September 2008 - May 2011

Led off-campus, multi-day outdoor trips for groups of 10+ participants. Adventures included caving, sea kayaking, backpacking, and rock climbing. Facilitated team building through challenge and high ropes courses.

\section{Mental Health Department Intern}

June 2009 - August 2009

Maryland Department of Mental Health \& Hygiene; Laurel, MD

Supervisor: Debra Hammond, LCSW-C; Office of Forensic Services

Developed and delivered report of 10 years of Maryland metal health data. 


\section{BARRIERS AND FACILITATORS OF SEEKING PSYCHOLOGICAL HELP}

Capital Partners for Education; Washington, D.C.

Forged partnerships with local schools to develop educational programs for underprivileged students. Aided in planning and implementation of student and mentor retreat.

Vice President

May 2008 - May 2010

National Society of Collegiate Scholars; University of Maryland, College Park, MD

Student Advisory Board Member

October 2007 - May 2009

Living and Learning Scholar's Program; University of Maryland, College Park, MD

TRAININGS

ATTENDED

\section{HONORS \&}

AWARDS

Society of Counseling Psychology
Div. 17 Section for Positive Psychology

Div. 17 Section on College and University Counseling Centers

West Virginia Psychological Association

Maryland Psychological Association of Graduate Students

American Counseling Association

Society for the Psychology of Women (Division 35)
Clinical Grant to serve on Mental Health Team at

World Scout Jamboree $(\$ 1,800)$

Herman L. Moses Merit Student Life Scholarship $(\$ 1,000)$

CEHS Student Research and Travel (\$500)

Woodrow \& Virginia Bonds Endowed Scholarship $(\$ 1,000)$

Omicron Delta Kappa, Towson University

Psi Chi, Psychology Honor Society

National Society of Collegiate Scholars

College Park Scholar's Program

Outstanding Trip Leader Award
March 2019

March 2019

September 2016

March 2016
July 2019

August 2018

March 2018

October 2017

March 2017

August 2016

March 2016

May 2015

May 2014 - present

May 2010 - present

May 2008 - present

Sept 2007 - May 2009

May 2011

2014 - present

2014 - present

2015 - present

2018 - present

$2015-2016$

$2014-2015$

$2013-2014$

$2014-2015$

\section{ASSESSMENT}

COMPETENCIES Beck Depression Inventory - II

Beck Anxiety Inventory

Behavior Assessment System for Children, Third Edition

Brief Symptom Inventory 18 (BSI-18)

Delis-Kaplan Executive Functioning Inventory

Integrated Visual and Auditory Continuous Performance Test (IVA-2)

Nonverbal Validity Profile Indicator

Pittsburgh Sleep Quality Index (PSQI)

Wechsler Adult Intelligence Scale - IV

Wechsler Abbreviated Scale of Intelligence - Second Edition

Woodcock Johnson - IV 
BARRIERS AND FACILITATORS OF SEEKING PSYCHOLOGICAL HELP

Trauma Screening Form - Child

Trauma Screening Form - Adult

DSM-5 Cross-Cutting Symptom Measure - Adult 